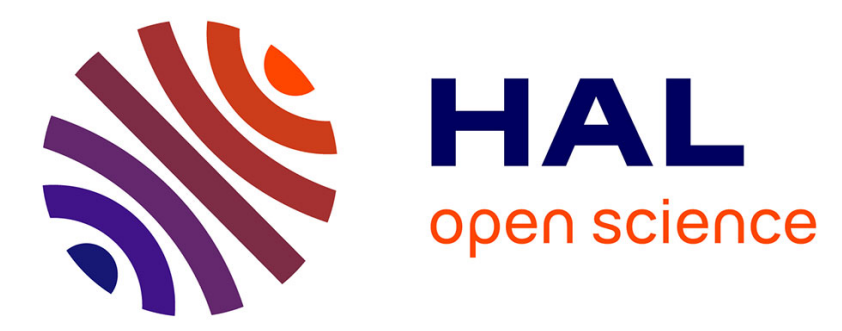

\title{
Isogeometric analysis of fiber reinforced composites using Kirchhoff-Love shell elements
}

J. Schulte, M. Dittmann, S. R. Eugster, S. Hesch, T. Reinicke, F. Dell'Isola, C. Hesch

\section{- To cite this version:}

J. Schulte, M. Dittmann, S. R. Eugster, S. Hesch, T. Reinicke, et al.. Isogeometric analysis of fiber reinforced composites using Kirchhoff-Love shell elements. Computer Methods in Applied Mechanics and Engineering, 2020, 362, pp.112845. hal-02915060

\section{HAL Id: hal-02915060 \\ https://hal.science/hal-02915060}

Submitted on 13 Aug 2020

HAL is a multi-disciplinary open access archive for the deposit and dissemination of scientific research documents, whether they are published or not. The documents may come from teaching and research institutions in France or abroad, or from public or private research centers.
L'archive ouverte pluridisciplinaire HAL, est destinée au dépôt et à la diffusion de documents scientifiques de niveau recherche, publiés ou non, émanant des établissements d'enseignement et de recherche français ou étrangers, des laboratoires publics ou privés. 


\title{
Isogeometric analysis of fiber reinforced composites using Kirchhoff-Love shell elements
}

\author{
J. Schulte ${ }^{\mathrm{a}}$, M. Dittmann ${ }^{\mathrm{a}}$, S.R. Eugster ${ }^{\mathrm{c}}$, S. Hesch ${ }^{\mathrm{b}}$, T. Reinicke ${ }^{\mathrm{b}}$, F. dell' Isola ${ }^{\mathrm{d}}$, C. Hesch $^{\mathrm{a}}$ \\ ${ }^{\text {a } C h a i r ~ o f ~ C o m p u t a t i o n a l ~ M e c h a n i c s, ~ U n i v e r s i t y ~ o f ~ S i e g e n, ~ S i e g e n, ~ G e r m a n y ~}$ \\ ${ }^{\mathrm{b}}$ Chair of Product Development, University of Siegen, Siegen, Germany \\ ${ }^{\mathrm{c}}$ Institute for Nonlinear Mechanics, University of Stuttgart, Stuttgart, Germany \\ ${ }^{\mathrm{d}}$ Department of Structural and Geotechnical Engineering, Sapienza University of Rome, Rome, Italy
}

\begin{abstract}
A second gradient theory for woven fabrics is applied to Kirchhoff-Love shell elements to analyze the mechanics of fiber reinforced composite materials. In particular, we assume a continuous distribution of the fibers embedded into the shell surface, accounting for additional in-plane flexural resistances $w$ ithin the h yperelastic r egime. For the fi nite el ement di scretization we apply isogeometric methods, i.e. we make use of B-splines as basis functions omitting the usage of mixed approaches. The higher gradient formulation of the fabric is verified by a s eries of $\mathrm{n}$ umerical examples, followed b y s uitable $\mathrm{v}$ alidation steps using experimental measurements on organic sheets. A final e xample u sing a $\mathrm{n}$ on-flat re ference ge ometry de monstrates the capabilities of the presented formulation.
\end{abstract}

Keywords: Second gradient elasticity; Woven fabrics; Isogeometric analysis

\section{Introduction}

\subsection{Strain gradient continua and fabric materials}

Classical Cauchy continuum formulations have been extended in a variational consistent manner towards general higher gradient theories in the pioneering and most fundamental work of Mindlin [1], see also the work of Germain [2] and Toupin [3,4] as well as Eringen [5-7]. A series of publications establish a link between the microstructure and the macroscopic formulation as generalized continua, see [8-12] among others, see also [13-17] for more details on the mathematical structure. Specific mechanical problems like elastic nets have been addressed by Steigmann et al. [18-21], for application on panthographic structures see dell'Isola et al. [22,23].

In this contribution we focus on fiber reinforced materials. The constitutive relation of this class of materials is governed by the given microstructure. In this context, we consider the length scales of the embedded fibers as small compared to the surrounding continuum, such that the terminus "microstructure" can be justified here. 


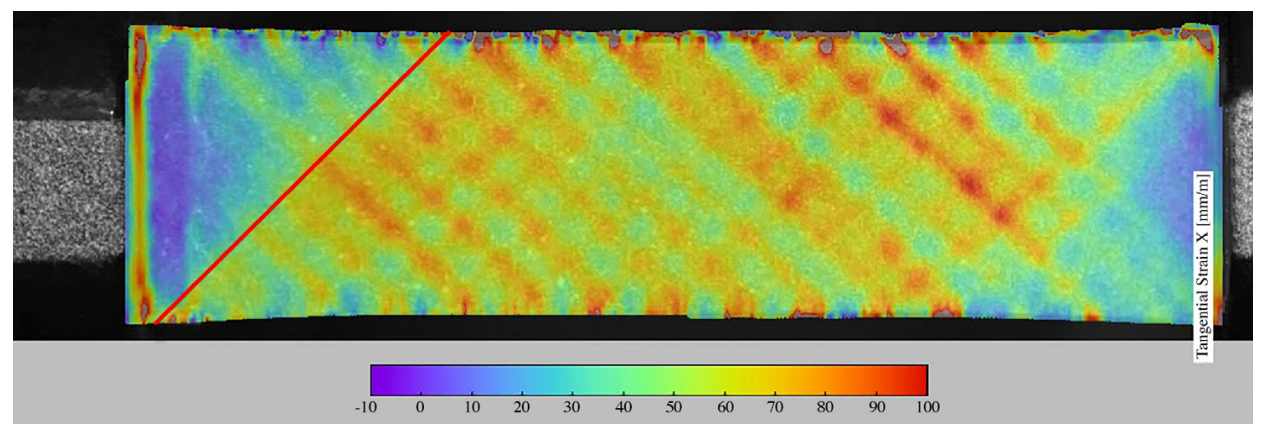

Fig. 1. DIC measurement of a 25 [mm] specimen in $45^{\circ}$ configuration, colors indicate local stretches in horizontal direction in [mm/m]. (For interpretation of the references to color in this figure legend, the reader is referred to the web version of this article.)

In particular, we embed a model of a woven fabric as presented in the work of Steigmann [21] to account for the in- and out-of-plane flexural resistances of the fibers in addition to the first gradient anisotropic effects of the fibers. To obtain the corresponding constitutive information, classical homogenization techniques are often applied, usually restricted to the first gradient anisotropic contributions. In particular, full field homogenizations are usually based on a finite element discretized unit cell (see, e.g. in Fish [24] for a comprehensive review on multiscale methods) and have been applied to composites, among many others, in Wang et al. [25], see also the references in Mishnaevsky [26]. Basic ideas on the homogenization of continua with micromorphic mesostructure can be found in the Hirschberger [27]. Current investigations on homogenization of continua with micromorphic attributes can be found in Hütter [28].

The woven fabric is embedded in a thin sheet of matrix material and thus, it is apparent to embed the fabric in a shell formulation. To be specific, we show that the formulation of the woven fabric as proposed by Steigmann can be embedded directly in the Kirchhoff-Love shell theory, such that the in-plane flexural resistances of the fibers is taken into account. Further discussions on higher-order contributions for the constitutive modeling of composites are presented in Asmanoglo \& Menzel [29,30], using the framework as provided in the preliminary work of Spencer \& Soldatos [31] and Soldatos [32].

B-spline based shape functions are employed for the spatial discretization, emanating from the field of Computer Aided Design (CAD). The corresponding finite element framework is widely known as isogeometric analysis (IGA), see Hughes et al. [33] and Cottrell et al. [34]. IGA allows to control the differentiability of the shape functions and thus, is suitable for higher gradient continua, see Fischer et al. [35] for applications on strain gradient elasticity. Here, we focus on Kirchhoff-Love shell formulations, analyzed in recent years using the concept of IGA, see, among many others, Kiendl et al. [36,37]. For general aspects including convergence results on strain gradient Kirchhoff-Love shells see Balobanov et al. [38].

\subsection{Material under investigation}

The numerical model proposed in this contribution has to be verified using suitable analytical results and validated using experimental data. In particular, the applicability of the numerical model will be demonstrated using a prototypical thermoplastic composite laminate, also known under the terminus organic sheet. Fig. 1 shows a digital image correlation (DIC), where we can observe strain gradient effects. In particular, the stiffness of the fibers against in-plane bending gives rise to specific deformation pattern, since the in-plane bending term results in an additional penalization on any change of the fiber flexure, as can be observed in the patterns of the DIC measurement compared to the straight red line drawn into the figure. Similar effects have been investigated intensively for pantographic structure, see, e.g. dell'Isola et al. [22,23] and references therein.

Since the organic sheets are small in thickness direction compared to its length and width, we consider a Kirchhoff-Love like shell formulation. The kinematics of this particular formulation focusing on additional deformation measures for the second gradient material model is introduced in Section 2. Based on this extended kinematic, the variational formulation is presented in Section 3. Within this section, the strain energies, stress resultants and the subsequent localization along with the arising boundary conditions are derived, which completes 
the model. The spatial discretization is given in Section 4, followed by numerical examples in Section 5. We first investigate in this section suitable verification steps of the numerical framework to ensure that the isogeometrical analysis is capable to reproduce certain analytical results. Afterwards, the model is validated against experimental results of the organic sheet. Eventually, conclusions are drawn in Section 6.

\section{Shell kinematics}

We consider the fiber reinforced composite as a three-dimensional body, whose points in the Euclidean threespace $\mathbb{E}^{3}$ are addressed by the convected coordinates $\left(\theta^{1}, \theta^{2}, \theta^{3}\right) \in \bar{B} \subset \mathbb{R}^{3}$. The reference placement $\boldsymbol{X}: B_{0} \rightarrow \mathbb{E}^{3}$ is the embedding ${ }^{1}$

$$
\boldsymbol{X}\left(\theta^{i}\right)=\overline{\boldsymbol{r}}\left(\theta^{\alpha}\right)+\theta^{3} \boldsymbol{N}\left(\theta^{\alpha}\right),
$$

where $\overline{\boldsymbol{r}}:=\overline{\boldsymbol{r}}\left(\theta^{\alpha}\right)$ is the parametrization of the reference mid-surface $\Omega \subset \mathbb{E}^{3}$, which comes along with the covariant basis $\boldsymbol{A}_{\alpha}$ and the unit normal vector field $\boldsymbol{N}$ defined by

$$
\boldsymbol{A}_{\alpha}:=\overline{\boldsymbol{r}}_{, \alpha} \quad \text { and } \quad \boldsymbol{N}:=\frac{\boldsymbol{A}_{1} \times \boldsymbol{A}_{2}}{\left\|\boldsymbol{A}_{1} \times \boldsymbol{A}_{2}\right\|},
$$

where $\overline{\boldsymbol{r}}_{, \alpha}:=\frac{\partial \overline{\boldsymbol{r}}}{\partial \theta^{\alpha}}$. Note that common operations defined on $\mathbb{E}^{3}$ are extended for vector and tensor fields in a pointwise fashion. For instance, the scalar field $\left\|\boldsymbol{A}_{1} \times \boldsymbol{A}_{2}\right\|$ is determined by $\left\|\boldsymbol{A}_{1} \times \boldsymbol{A}_{2}\right\|\left(\theta^{\alpha}\right):=\left\|\boldsymbol{A}_{1}\left(\theta^{\alpha}\right) \times \boldsymbol{A}_{2}\left(\theta^{\alpha}\right)\right\|$, where we applied the cross product $\times$ of $\mathbb{E}^{3}$ together with the standard Euclidean norm $\|\boldsymbol{v}\|:=\sqrt{(\boldsymbol{v} \cdot \boldsymbol{v})}, \boldsymbol{v} \in \mathbb{E}^{3}$ induced by the inner product $\cdot$ of $\mathbb{E}^{3}$. In the reference placement (1), the coordinates are chosen such that $\theta^{3} \in[-h / 2, h / 2]$ denotes the coordinate in thickness direction with $h$ as the thickness of the body.

The deformation of the body is described by the function $x: B \rightarrow \mathbb{E}^{3}$, which incorporates the Kirchhoff hypothesis by the kinematical ansatz

$$
\boldsymbol{x}\left(\theta^{i}\right)=\boldsymbol{r}\left(\theta^{\alpha}\right)+\theta^{3} \boldsymbol{n}\left(\theta^{\alpha}\right) .
$$

Here, $\boldsymbol{r}:=\boldsymbol{r}\left(\theta^{\alpha}\right)$ is the parametrization of the deformed mid-surface $\omega \subset \mathbb{E}^{3}$, which comes along with the covariant basis $\boldsymbol{a}_{\alpha}$ and the unit normal vector field $\boldsymbol{n}$ defined by

$$
\boldsymbol{a}_{\alpha}=\boldsymbol{r}_{, \alpha} \quad \text { and } \quad \boldsymbol{n}=\frac{\boldsymbol{a}_{1} \times \boldsymbol{a}_{2}}{\left\|\boldsymbol{a}_{1} \times \boldsymbol{a}_{2}\right\|} .
$$

The kinematical restriction (3) allows to describe the body's deformation exclusively by the geometry of the midsurface $\omega$. Therefore, we introduce some important geometric objects that play a central role in the development of the mechanical theory. For a concise treatment of surface geometry, we refer to [39]. The co- and contravariant metric coefficients of $\omega$ are defined by

$$
a_{\alpha \beta}:=\boldsymbol{a}_{\alpha} \cdot \boldsymbol{a}_{\beta} \quad \text { and } \quad\left[a^{\alpha \beta}\left(\theta^{\alpha}\right)\right]:=\left[a_{\alpha \beta}\left(\theta^{\alpha}\right)\right]^{-1},
$$

where the square brackets indicate the component matrix. Consequently, it holds that $a_{\alpha \beta} a^{\beta \mu}=\delta_{\alpha}^{\mu}$. The contravariant basis $\boldsymbol{a}^{\alpha}$ is introduced by the relation $\boldsymbol{a}^{\alpha} \cdot \boldsymbol{a}_{\beta}=\delta_{\beta}^{\alpha}$ and can therefore be expressed as $\boldsymbol{a}^{\alpha}=a^{\alpha \beta} \boldsymbol{a}_{\beta}$, which in turn leads to $a^{\alpha \beta}=\boldsymbol{a}^{\alpha} \cdot \boldsymbol{a}^{\beta}$. By definition of the cross-product, we can rewrite $\left\|\boldsymbol{a}_{1} \times \boldsymbol{a}_{2}\right\|^{2}=\left[\left(\boldsymbol{a}_{1} \cdot \boldsymbol{a}_{1}\right)\left(\boldsymbol{a}_{2} \cdot \boldsymbol{a}_{2}\right)-\left(\boldsymbol{a}_{1} \cdot \boldsymbol{a}_{2}\right)^{2}\right]=$ $\operatorname{det}\left(a_{\alpha \beta}\right)$. The partial derivatives of the covariant base vectors and the normal vectors can be expressed using the Gauss and Weingarten equations

$$
\boldsymbol{r}_{, \alpha \beta}=\boldsymbol{a}_{\alpha, \beta}=\Gamma_{\alpha \beta}^{\sigma} \boldsymbol{a}_{\sigma}+b_{\alpha \beta} \boldsymbol{n} \quad \text { and } \quad \boldsymbol{n}_{, \alpha}=-b_{\alpha \beta} \boldsymbol{a}^{\beta},
$$

where we use the Christoffel symbols of the second kind $\Gamma_{\alpha \beta}^{\sigma}$ and the covariant components of the curvature tensor $b_{\alpha \beta}$ defined by

$$
\Gamma_{\alpha \beta}^{\sigma}:=\boldsymbol{a}^{\sigma} \cdot \boldsymbol{a}_{\alpha, \beta} \quad \text { and } \quad b_{\alpha \beta}:=\boldsymbol{a}_{\alpha, \beta} \cdot \boldsymbol{n} .
$$

Note that $(6)_{1}$ can also be expressed as

$$
\boldsymbol{a}_{\alpha, \beta}=\Gamma_{\alpha \beta \sigma} \boldsymbol{a}^{\sigma}+b_{\alpha \beta} \boldsymbol{n}
$$

\footnotetext{
1 Greek and Latin indices range in the sets $\{1,2\}$ and $\{1,2,3\}$, respectively. Moreover, we will apply Einstein summation convention for repeated upper and lower indices.
} 
where

$$
\Gamma_{\alpha \beta \sigma}=\boldsymbol{a}_{\sigma} \cdot \boldsymbol{a}_{\alpha, \beta}=\boldsymbol{a}^{\lambda} \cdot \boldsymbol{a}_{\alpha, \beta} a_{\lambda \sigma}=\Gamma_{\alpha \beta}^{\lambda} a_{\lambda \sigma}
$$

denotes the Christoffel symbols of the first kind. Analogously, we can define the above introduced objects and relations also for the reference mid-surface $\Omega$ using the co- and contravariant metric coefficients $A_{\alpha \beta}$ and $A^{\alpha \beta}$, the contravariant base vectors $\boldsymbol{A}^{\alpha}$, the Christoffel symbols of the second kind $\bar{\Gamma}_{\alpha \beta}^{\sigma}$, as well as the covariant components of the curvature tensor $B_{\alpha \beta}$.

The surface-deformation gradient $\boldsymbol{F}$, defined by $\mathrm{d} \boldsymbol{r}=\boldsymbol{F} \mathrm{d} \overline{\boldsymbol{r}}$, can be computed by inserting $\mathrm{d} \theta^{\alpha}=\mathrm{d} \theta^{\beta} \boldsymbol{A}_{\beta} \cdot \boldsymbol{A}^{\alpha}=$ $\overline{\boldsymbol{r}}_{, \beta} \mathrm{d} \theta^{\beta} \cdot \boldsymbol{A}^{\alpha}=\mathrm{d} \overline{\boldsymbol{r}} \cdot \boldsymbol{A}^{\alpha}$ into the expression $\mathrm{d} \boldsymbol{r}=\boldsymbol{r},{ }_{\alpha} \mathrm{d} \theta^{\alpha}=\boldsymbol{a}_{\alpha}\left(\mathrm{d} \overline{\boldsymbol{r}} \cdot \boldsymbol{A}^{\alpha}\right)=\left(\boldsymbol{a}_{\alpha} \otimes \boldsymbol{A}^{\alpha}\right) \mathrm{d} \overline{\boldsymbol{r}}$ and is consequently given by

$$
\boldsymbol{F}=\boldsymbol{a}_{\alpha} \otimes \boldsymbol{A}^{\alpha}
$$

The covariant base vectors $\boldsymbol{g}_{i}=\boldsymbol{x}_{, i}$ of the three-dimensional body in the deformed configuration are

$$
\boldsymbol{g}_{\alpha}\left(\theta^{i}\right)=\boldsymbol{a}_{\alpha}\left(\theta^{\alpha}\right)+\theta^{3} \boldsymbol{n}_{, \alpha}\left(\theta^{\alpha}\right) \quad \text { and } \quad \boldsymbol{g}_{3}\left(\theta^{i}\right)=\boldsymbol{n}\left(\theta^{\alpha}\right)
$$

and $\boldsymbol{G}_{i}=\boldsymbol{X}_{, i}$ in the reference configuration

$$
\boldsymbol{G}_{\alpha}\left(\theta^{i}\right)=\boldsymbol{A}_{\alpha}\left(\theta^{\alpha}\right)+\theta^{3} \boldsymbol{N}_{, \alpha}\left(\theta^{\alpha}\right) \quad \text { and } \quad \boldsymbol{G}_{3}\left(\theta^{i}\right)=\boldsymbol{N}\left(\theta^{\alpha}\right) .
$$

The covariant metric coefficients $g_{i j}=\boldsymbol{g}_{i} \cdot \boldsymbol{g}_{j}$ of the three-dimensional body in the deformed configuration are determined as

$$
\begin{aligned}
& g_{\alpha \beta}\left(\theta^{i}\right)=a_{\alpha \beta}\left(\theta^{\alpha}\right)-2 \theta^{3} b_{\alpha \beta}\left(\theta^{\alpha}\right)+\left(\theta^{3}\right)^{2} \boldsymbol{n}_{, \alpha}\left(\theta^{\alpha}\right) \cdot \boldsymbol{n}_{, \beta}\left(\theta^{\alpha}\right), \\
& g_{\alpha 3}\left(\theta^{i}\right)=g_{3 \alpha}\left(\theta^{i}\right)=0, \\
& g_{33}\left(\theta^{i}\right)=1,
\end{aligned}
$$

where we applied the definitions (5) and (6) $)_{2}$ together with the properties $\boldsymbol{n} \cdot \boldsymbol{n}=1, \boldsymbol{a}_{\alpha} \cdot \boldsymbol{n}=0$ and $\boldsymbol{n}_{, \alpha} \cdot \boldsymbol{n}=0$. Analogously, we obtain the covariant metric coefficients $G_{i j}=\boldsymbol{G}_{i} \cdot \boldsymbol{G}_{j}$ in the reference configuration

$$
\begin{aligned}
& G_{\alpha \beta}\left(\theta^{i}\right)=A_{\alpha \beta}\left(\theta^{\alpha}\right)-2 \theta^{3} B_{\alpha \beta}\left(\theta^{\alpha}\right)+\left(\theta^{3}\right)^{2} \boldsymbol{N}_{, \alpha}\left(\theta^{\alpha}\right) \cdot \boldsymbol{N}_{, \beta}\left(\theta^{\alpha}\right), \\
& G_{\alpha 3}\left(\theta^{i}\right)=G_{3 \alpha}\left(\theta^{i}\right)=0, \\
& G_{33}\left(\theta^{i}\right)=1 .
\end{aligned}
$$

Neglecting the quadratic term in Eqs. (13) and (14) yields

$$
g_{\alpha \beta}\left(\theta^{i}\right)=a_{\alpha \beta}\left(\theta^{\alpha}\right)-2 \theta^{3} b_{\alpha \beta}\left(\theta^{\alpha}\right) \quad \text { and } \quad G_{\alpha \beta}\left(\theta^{i}\right)=A_{\alpha \beta}\left(\theta^{\alpha}\right)-2 \theta^{3} B_{\alpha \beta}\left(\theta^{\alpha}\right)
$$

assuming a linear behavior through the thickness of the body. Due to the block structure of the metric coefficient matrices, the contravariant base vectors $\boldsymbol{g}^{i}$ and $\boldsymbol{G}^{i}$ in the deformed and the reference configuration can be computed as

$$
\boldsymbol{g}^{\alpha}=g^{\alpha \beta} \boldsymbol{g}_{\beta}, \quad \boldsymbol{g}^{3}=\boldsymbol{g}_{3}=\boldsymbol{n}, \quad\left[g^{\alpha \beta}\left(\theta^{i}\right)\right]=\left[g_{\alpha \beta}\left(\theta^{i}\right)\right]^{-1},
$$

and

$$
\boldsymbol{G}^{\alpha}=G^{\alpha \beta} \boldsymbol{G}_{\beta}, \quad \boldsymbol{G}^{3}=\boldsymbol{G}_{3}=\boldsymbol{N}, \quad\left[G^{\alpha \beta}\left(\theta^{i}\right)\right]=\left[G_{\alpha \beta}\left(\theta^{i}\right)\right]^{-1},
$$

respectively. Similar to the surface-deformation gradient $\boldsymbol{F}$, the deformation gradient of the three-dimensional body $\hat{\boldsymbol{F}}$ is

$$
\hat{\boldsymbol{F}}=\boldsymbol{g}_{i} \otimes \boldsymbol{G}^{i}
$$

which is easily obtained by $\mathrm{d} \boldsymbol{x}=\boldsymbol{x}_{, i} \mathrm{~d} \theta^{i}=\boldsymbol{g}_{i}\left(\mathrm{~d} \boldsymbol{X} \cdot \boldsymbol{G}^{i}\right)=\left(\boldsymbol{g}_{i} \otimes \boldsymbol{G}^{\boldsymbol{i}}\right) \mathrm{d} \boldsymbol{X}=\hat{\boldsymbol{F}} \mathrm{d} \boldsymbol{X}$.

\subsection{Deformation measures}

The right Cauchy-Green deformation tensor for the surface is

$$
\boldsymbol{C}:=\boldsymbol{F}^{T} \boldsymbol{F}=C_{\alpha \beta} \boldsymbol{A}^{\alpha} \otimes \boldsymbol{A}^{\beta},
$$


where we have defined $C_{\alpha \beta}\left(a_{\alpha \beta}\left(\theta^{\alpha}\right)\right):=a_{\alpha \beta}\left(\theta^{\alpha}\right)$. Introducing the difference

$$
S_{\alpha \beta}^{\lambda}:=\Gamma_{\alpha \beta}^{\lambda}-\bar{\Gamma}_{\alpha \beta}^{\lambda} \quad \text { and } \quad S_{\alpha \beta \lambda}:=S_{\alpha \beta}^{\sigma} a_{\sigma \lambda}=\Gamma_{\alpha \beta \lambda}-\bar{\Gamma}_{\alpha \beta}^{\sigma} a_{\sigma \lambda},
$$

it is convenient to define the second covariant derivative of the deformation with respect to the reference configuration as

$$
\begin{aligned}
\boldsymbol{r}_{\mid \alpha \beta} & :=\boldsymbol{r}_{, \alpha \beta}-\bar{\Gamma}_{\alpha \beta}^{\sigma} \boldsymbol{a}_{\sigma}=\left(\Gamma_{\alpha \beta}^{\sigma}-\bar{\Gamma}_{\alpha \beta}^{\sigma}\right) \boldsymbol{a}_{\sigma}+b_{\alpha \beta} \boldsymbol{n} \\
& =S_{\alpha \beta}^{\sigma} \boldsymbol{a}_{\sigma}+b_{\alpha \beta} \boldsymbol{n}=S_{\alpha \beta \sigma} \boldsymbol{a}^{\sigma}+b_{\alpha \beta} \boldsymbol{n} .
\end{aligned}
$$

Thus, we obtain

$$
\left(\boldsymbol{r}_{\mid \alpha \beta}-B_{\alpha \beta} \boldsymbol{n}\right) \otimes \boldsymbol{A}^{\alpha} \otimes \boldsymbol{A}^{\beta}=\boldsymbol{a}_{\sigma} \otimes \boldsymbol{S}^{\sigma}-\boldsymbol{n} \otimes \boldsymbol{\kappa}
$$

with

$$
\boldsymbol{S}^{\sigma}:=S_{\alpha \beta}^{\sigma} \boldsymbol{A}^{\alpha} \otimes \boldsymbol{A}^{\beta} \quad \text { and } \quad \boldsymbol{\kappa}:=\kappa_{\alpha \beta} \boldsymbol{A}^{\alpha} \otimes \boldsymbol{A}^{\beta}=\left(B_{\alpha \beta}-b_{\alpha \beta}\right) \boldsymbol{A}^{\alpha} \otimes \boldsymbol{A}^{\beta},
$$

where in-plane and out-of-plane curvatures can be addressed by eight and four components, respectively. By the definitions (5) and (7), it can readily be seen that $a_{\alpha \beta}, S_{\alpha \beta}^{\sigma}$ and $\kappa_{\alpha \beta}$ are invariant under superimposed rigid body motions, a proof can be found in Appendix A.

In the following, we will introduce some deformation measures for the embedded fibers which all can be considered as functions of $a_{\alpha \beta}, S_{\alpha \beta}^{\sigma}, \kappa_{\alpha \beta}$ and $\theta^{\alpha}$. An extensive discussion about these fiber deformation measures can be found in Appendix B following Steigmann and dell'Isola [20]. For the two families of embedded fibers, which are characterized by their constant orthogonal vector fields in the reference configuration $\boldsymbol{M}$ and $\boldsymbol{L}$, the squared fiber stretches are given by

$$
\lambda_{1}^{2}:=\frac{a_{\alpha \beta} L^{\alpha} L^{\beta}}{A_{\mu \nu} L^{\mu} L^{\nu}} \quad \text { and } \quad \lambda_{2}^{2}:=\frac{a_{\mu \nu} M^{\mu} M^{\nu}}{A_{\mu \nu} M^{\mu} M^{\nu}} .
$$

The change in the angle between the fibers is given by

$$
\varphi:=\operatorname{acos}\left(\frac{A_{\alpha \beta} L^{\alpha} M^{\beta}}{\sqrt{A_{\mu \nu} L^{\mu} L^{\nu} A_{\sigma \rho} M^{\sigma} M^{\rho}}}\right)-\operatorname{acos}\left(\frac{a_{\alpha \beta} L^{\alpha} M^{\beta}}{\sqrt{a_{\mu \nu} L^{\mu} L^{\nu} a_{\sigma \rho} M^{\sigma} M^{\rho}}}\right) .
$$

Moreover,

$$
K_{1}:=-\frac{\kappa_{\alpha \beta} L^{\alpha} L^{\beta}}{A_{\mu \nu} L^{\mu} L^{\nu}}, \quad K_{2}:=-\frac{\kappa_{\alpha \beta} M^{\alpha} M^{\beta}}{A_{\mu \nu} M^{\mu} M^{\nu}} \quad \text { and } \quad K_{3}:=-\frac{\kappa_{\alpha \beta} L^{\alpha} M^{\beta}}{\sqrt{A_{\mu \nu} L^{\mu} L^{\nu} A_{\sigma \rho} M^{\sigma} M^{\rho}}},
$$

representing the out-of-plane bending and twist of the fibers. Finally,

$$
\boldsymbol{g}_{L}:=\frac{L^{\alpha} L^{\beta} S_{\alpha \beta \sigma}}{A_{\mu \nu} L^{\mu} L^{\nu}} \boldsymbol{a}^{\sigma}, \quad \boldsymbol{g}_{M}:=\frac{M^{\alpha} M^{\beta} S_{\alpha \beta \sigma}}{A_{\mu \nu} M^{\mu} M^{\nu}} \boldsymbol{a}^{\sigma} \quad \text { and } \quad \boldsymbol{\Gamma}:=\frac{L^{\alpha} M^{\beta} S_{\alpha \beta \sigma}}{\sqrt{A_{\mu \nu} L^{\mu} L^{\nu} A_{\sigma \rho} M^{\sigma} M^{\rho}}} \boldsymbol{a}^{\sigma},
$$

represent the combined effects of gradients of the fiber stretch and in-plane bending.

For the matrix material, the right Cauchy-Green tensor reads

$$
\hat{\boldsymbol{C}}=\hat{\boldsymbol{F}}^{T} \hat{\boldsymbol{F}}=g_{i j} \boldsymbol{G}^{i} \otimes \boldsymbol{G}^{j},
$$

whose covariant components coincide with the metric components of Eq. (13); note that higher order terms in $\theta^{3}$ are neglected. Due to the dependence of the metric coefficients (15) on the first and second fundamental form, it is convenient to introduce the components of the right Cauchy-Green tensor as $\hat{C}_{i j}\left(a_{\alpha \beta}\left(\theta^{\alpha}\right), \kappa_{\alpha \beta}\left(\theta^{\alpha}\right), \theta^{3}\right)=g_{i j}\left(\theta^{i}\right)$. The kinematical restriction in Eq. (3) does not allow for any change in thickness direction. For a fixed $\theta^{3}=\tilde{\theta}^{3}$, the surface elements of the embedded surfaces in the reference and the deformed configurations $\boldsymbol{X}\left(\theta^{\alpha}, \tilde{\theta}^{3}\right)$ and $\boldsymbol{x}\left(\theta^{\alpha}, \tilde{\theta}^{3}\right)$, respectively, are

$$
\begin{aligned}
\mathrm{d} a\left(\theta^{\alpha}, \tilde{\theta}^{3}\right) & =\left\|\boldsymbol{g}_{1}\left(\theta^{\alpha}, \tilde{\theta}^{3}\right) \times \boldsymbol{g}_{2}\left(\theta^{\alpha}, \tilde{\theta}^{3}\right)\right\| \mathrm{d} \theta^{1} \mathrm{~d} \theta^{2}=\sqrt{\operatorname{det}\left(g_{\alpha \beta}\left(\theta^{\alpha}, \tilde{\theta}^{3}\right)\right)} \mathrm{d} \theta^{1} \mathrm{~d} \theta^{2}, \\
\mathrm{~d} A\left(\theta^{\alpha}, \tilde{\theta}^{3}\right) & =\left\|\boldsymbol{G}_{1}\left(\theta^{\alpha}, \tilde{\theta}^{3}\right) \times \boldsymbol{G}_{2}\left(\theta^{\alpha}, \tilde{\theta}^{3}\right)\right\| \mathrm{d} \theta^{1} \mathrm{~d} \theta^{2}=\sqrt{\operatorname{det}\left(G_{\alpha \beta}\left(\theta^{\alpha}, \tilde{\theta}^{3}\right)\right)} \mathrm{d} \theta^{1} \mathrm{~d} \theta^{2} .
\end{aligned}
$$


Consequently, the in-plane Jacobian determinant

$$
\hat{J}_{0}\left(\theta^{i}\right):=\sqrt{\operatorname{det}\left(\hat{\boldsymbol{C}}\left(\theta^{i}\right)\right)}=\sqrt{\frac{\operatorname{det}\left(g_{\alpha \beta}\left(\theta^{\alpha}, \theta^{3}\right)\right)}{\operatorname{det}\left(G_{\alpha \beta}\left(\theta^{\alpha}, \theta^{3}\right)\right)}}
$$

can be interpreted as the areal dilatation, i.e. the ratio between the deformed and the reference area elements $\mathrm{d} a$ and $\mathrm{d} A$, respectively.

\section{Variational formulation}

For the matrix material we assume the strain energy function per unit reference volume $\Psi^{\text {iso }}\left(\hat{C}_{i j}, \theta^{i}\right)$, for the fibers per unit reference area $\Psi^{\mathrm{fib}}\left(a_{\alpha \beta}, S_{\alpha \beta \sigma}, \kappa_{\alpha \beta}, \theta^{\alpha}\right)$. Moreover, we assume that the embedded fibers have a height of $h_{\mathrm{f}}$ such that the space occupied by matrix material in the reference configuration is defined by $\left\{\boldsymbol{X}\left(\theta^{i}\right) \mid \theta^{3} \in \varpi_{\text {iso }}\right\}$, where $\varpi_{\text {iso }}=\left[-\frac{h}{2}, \frac{h}{2}\right] \backslash\left(-\frac{h_{\mathrm{f}}}{2}, \frac{h_{\mathrm{f}}}{2}\right)$. For the overall composite we obtain for the strain energy defined per unit reference area of the surface

$$
\Psi\left(a_{\alpha \beta}, S_{\alpha \beta \sigma}, \kappa_{\alpha \beta}, \theta^{\alpha}\right)=\int_{\varpi_{\text {iso }}} \Psi^{\text {iso }}\left(\hat{C}_{i j}\left(a_{\alpha \beta}, \kappa_{\alpha \beta}, \theta^{3}\right), \theta^{i}\right) \mathrm{d} \theta^{3}+\Psi^{\mathrm{fib}}\left(a_{\alpha \beta}, S_{\alpha \beta \sigma}, \kappa_{\alpha \beta}, \theta^{\alpha}\right),
$$

which allows us to write the internal energy as $W^{\text {int }}=\int_{\Omega} \Psi \mathrm{d} A$. For the finite element analysis, we will discretize the virtual work expression, which for the composite material reads as

$$
\delta W^{\text {int }}=\int_{\Omega}\left[\int_{\varpi_{\text {iso }}} \delta \Psi^{\text {iso }}\left(\hat{C}_{i j}\left(a_{\alpha \beta}, \kappa_{\alpha \beta}, \theta^{3}\right), \theta^{i}\right) \mathrm{d} \theta^{3}+\delta \Psi^{\mathrm{fib}}\left(a_{\alpha \beta}, S_{\alpha \beta \sigma}, \kappa_{\alpha \beta}, \theta^{\alpha}\right)\right] \mathrm{d} A .
$$

Noting that $\delta \hat{C}_{i j}=\delta_{i}^{\alpha} \delta_{j}^{\beta}\left(\delta a_{\alpha \beta}+2 \theta^{3} \delta \kappa_{\alpha \beta}\right)$ yields

$$
\delta W^{\mathrm{int}}=\int_{\Omega}\left[\left(n_{\mathrm{iso}}^{\alpha \beta}+n_{\mathrm{fib}}^{\alpha \beta}\right) \delta a_{\alpha \beta}+\left(m_{\mathrm{iso}}^{\alpha \beta}+m_{\mathrm{fib}}^{\alpha \beta}\right) \delta \kappa_{\alpha \beta}+m_{\mathrm{fib}}^{\alpha \beta \sigma} \delta S_{\alpha \beta \sigma}\right] \mathrm{d} A,
$$

where we have made use of a series of abbreviations for the stress resultants; a first set obtained by integration through thickness

$$
n_{\text {iso }}^{\alpha \beta}:=\int_{\varpi_{\text {iso }}} \frac{\partial \Psi^{\text {iso }}}{\partial \hat{C}_{\alpha \beta}} \mathrm{d} \theta^{3} \quad \text { and } \quad m_{\text {iso }}^{\alpha \beta}:=\int_{\varpi_{\text {iso }}} 2 \theta^{3} \frac{\partial \Psi^{\text {iso }}}{\partial \hat{C}_{\alpha \beta}} \mathrm{d} \theta^{3}
$$

and a second set for the fiber contributions

$$
n_{\mathrm{fib}}^{\alpha \beta}:=\frac{\partial \Psi^{\mathrm{fib}}}{\partial a_{\alpha \beta}}, \quad m_{\mathrm{fib}}^{\alpha \beta}:=\frac{\partial \Psi^{\mathrm{fib}}}{\partial \kappa_{\alpha \beta}} \quad \text { and } \quad m_{\mathrm{fib}}^{\alpha \beta \sigma}:=\frac{\partial \Psi^{\mathrm{fib}}}{\partial S_{\alpha \beta \sigma}}
$$

directly related to the mid-surface, see Appendix C for further details. The variations of $a_{\alpha \beta}, \kappa_{\alpha \beta}$ and $S_{\alpha \beta \sigma}$ can be expressed in terms of $\delta \boldsymbol{r}\left(\theta^{\alpha}\right)=\hat{\boldsymbol{r}}_{, \varepsilon}\left(\theta^{\alpha}, \varepsilon_{0}\right)$, which is the variational derivative of $\boldsymbol{r}^{2}$ induced by the one-parameter family $\hat{\boldsymbol{r}}\left(\theta^{\alpha}, \varepsilon\right)$ for which $\hat{\boldsymbol{r}}\left(\theta^{\alpha}, \varepsilon_{0}\right)=\boldsymbol{r}\left(\theta^{\alpha}\right)$ holds. The variation of the covariant base vectors as well as the partial derivatives thereof are $\delta \boldsymbol{a}_{\alpha}=\delta \boldsymbol{r}_{, \alpha}$ and $\delta \boldsymbol{a}_{\alpha, \beta}=\delta \boldsymbol{r}_{, \alpha \beta}$, respectively. The variation of the first fundamental form (5) can be written as

$$
\delta a_{\alpha \beta}=\boldsymbol{a}_{\alpha} \cdot \delta \boldsymbol{a}_{\beta}+\boldsymbol{a}_{\beta} \cdot \delta \boldsymbol{a}_{\alpha} .
$$

The variation of the out-of-plane curvature changes is due to (23) and (7) given by

$$
\delta \kappa_{\alpha \beta}=-\left(\delta \boldsymbol{a}_{\alpha, \beta} \cdot \boldsymbol{n}+\boldsymbol{a}_{\alpha, \beta} \cdot \delta \boldsymbol{n}\right),
$$

where the variation of the unit normal vector reads

$$
\delta \boldsymbol{n}=\frac{(\boldsymbol{I}-\boldsymbol{n} \otimes \boldsymbol{n})\left(\boldsymbol{a}_{1} \times \delta \boldsymbol{a}_{2}+\delta \boldsymbol{a}_{1} \times \boldsymbol{a}_{2}\right)}{\left\|\boldsymbol{a}_{1} \times \boldsymbol{a}_{2}\right\|}
$$

using the identity map $\boldsymbol{I}$. Eventually, the variation of the in-plane curvature (20) gives rise to

$$
\delta S_{\alpha \beta \sigma}=\delta \boldsymbol{a}_{\alpha, \beta} \cdot \boldsymbol{a}_{\sigma}+\boldsymbol{a}_{\alpha, \beta} \cdot \delta \boldsymbol{a}_{\sigma}-\bar{\Gamma}_{\alpha \beta}^{\lambda}\left(\boldsymbol{a}_{\lambda} \cdot \delta \boldsymbol{a}_{\sigma}+\boldsymbol{a}_{\sigma} \cdot \delta \boldsymbol{a}_{\lambda}\right) .
$$

\footnotetext{
2 For details on the functional space of the admissible test functions see end of Section 3.2.
} 


\subsection{Strain energies}

For the matrix material, we assume the existence of an incompressible neo-Hookean material, given by

$$
\Psi^{\text {iso }}=\frac{1}{2} \mu\left(\operatorname{tr}\left(\hat{C}_{\alpha \beta} \boldsymbol{G}^{\alpha} \otimes \boldsymbol{G}^{\beta}\right)+\left(\frac{1}{\hat{J}_{0}}\right)^{2}-3\right)=\frac{1}{2} \mu\left(\hat{C}_{\alpha \beta} G^{\alpha \beta}+\frac{\operatorname{det}\left(G_{\mu \nu}\right)}{\operatorname{det}\left(\hat{C}_{\alpha \beta}\right)}-3\right),
$$

with the shear modulus $\mu$. This strain energy function is obtained from its three-dimensional version by assuming a plane stress distribution in the three-dimensional continuum together with a decoupling of in-plane and thickness deformations. Note that this is a correction on a constitutive level for the too restrictive kinematical ansatz (3), which does not allow for thickness deformation.

Remark 1. For a compressible material model, the plane stress condition cannot be inserted analytically, i.e. we have to condense the corresponding conditions numerically. For details, we refer to Kiendl et al. [37].

Assuming rectangular cross-sections for the embedded fibers, we adopt the following strain energy function

$$
\Psi^{\mathrm{fib}}=\sum_{\alpha=1}^{2} \frac{a_{\alpha}}{2}\left(\lambda_{\alpha}-1\right)^{2}+\frac{a_{3}}{2} \tan (\varphi)^{2}+\sum_{i=1}^{3} \frac{k_{i}}{2}\left(K_{i}\right)^{2}+\frac{1}{2}\left(g_{1}\left(\boldsymbol{g}_{L} \cdot \boldsymbol{g}_{L}\right)+g_{2}\left(\boldsymbol{g}_{M} \cdot \boldsymbol{g}_{M}\right)+g_{3}(\boldsymbol{\Gamma} \cdot \boldsymbol{\Gamma})\right)
$$

where the constant material parameters $a_{i}$ are associated to the stretch and the change of angle between the fibers. In particular, $a_{\alpha}=E_{\alpha} h_{\mathrm{f}}$ correlates to Young's modulus $E_{\alpha}$ of the particular fiber, whereas $a_{3}=G h_{\mathrm{f}}$ represents the shear stiffness of a shell continuum. Note that the fiber formulation does not take any Poisson effect into account, i.e. the Poisson's ratio $v=0$ and thus, $G=\frac{1}{2} E$ in a classical continuum. In the present formulation, $G$ represents the stiffness against twist between the fibers and is in general independent of the fiber stiffness. The out-of-plane bending stiffness $k_{\alpha}$ depends on the particular microstructure of the embedded fibers, i.e. on the second moment of area, such that $k_{\alpha}=E_{\alpha} I_{\alpha} / b_{\alpha}$. Here, $b_{\alpha}$ denotes a representative in-plane length scale of the fiber, such that we obtain a bending stiffness of $k_{\alpha}=E_{\alpha} h_{\mathrm{f}}^{3} / 12$ for a continuously distributed fiber. Analogously, we obtain for the in-plane bending stiffness $g_{\alpha}=E_{\alpha} b_{\alpha}^{2} h_{\mathrm{f}} / 12$. Note that other microstructures are possible, although characteristics like fiber height and width have to be discussed and validated for the particular geometry. Eventually, the terms associated with $k_{3}$ and $g_{3}$ control the fiber torsion as well as the in-plane deformation captured by Tchebychev curvatures and strain gradients, see Appendix B.

Remark 2. Assuming that the same fibers are used in both directions, the same material parameters can be applied for both fibers, i.e. $a_{1}=a_{2}, k_{1}=k_{2}$ and $g_{1}=g_{2}$. Moreover, we note that the used strain measures of the fiber components associated with curvature terms account always for combined effects of the gradients of the fiber stretch and bending. Thus, the identification of the material parameters in terms of classical beam theory is limited, as we will show in Section 5.

\subsection{Localization}

To identify and collect the resulting bending moments and normal stress contributions, we reorganize the virtual work expression. First, we reorganize the variation of the curvature tensor as follows

$$
\begin{aligned}
\delta b_{\alpha \beta} & =\boldsymbol{n} \cdot \delta \boldsymbol{a}_{\alpha, \beta}+\boldsymbol{a}_{\alpha, \beta} \cdot \delta \boldsymbol{n}, \\
& =\boldsymbol{n} \cdot\left[\delta \boldsymbol{a}_{\alpha, \beta}-\Gamma_{\alpha \beta}^{\sigma} \delta \boldsymbol{a}_{\sigma}\right],
\end{aligned}
$$

where we make use of $\boldsymbol{a}_{\sigma} \cdot \delta \boldsymbol{n}=-\boldsymbol{n} \cdot \delta \boldsymbol{a}_{\sigma}$ and $\boldsymbol{n} \cdot \delta \boldsymbol{n}=0$ along with the Gauss and Weingarten relation (6) 1 . Taking the second covariant derivative introduced in (21) into account yields

$$
\delta b_{\alpha \beta}=\boldsymbol{n} \cdot\left[\delta \boldsymbol{r}_{\mid \alpha \beta}-S_{\alpha \beta}^{\sigma} \delta \boldsymbol{a}_{\sigma}\right] .
$$

Next, the covariant term $S_{\alpha \beta \sigma}=\left(\Gamma_{\alpha \beta}^{\lambda}-\bar{\Gamma}_{\alpha \beta}^{\lambda}\right) a_{\lambda \sigma}$ gives rise to

$$
\delta S_{\alpha \beta \sigma}=\delta \Gamma_{\alpha \beta \sigma}-\bar{\Gamma}_{\alpha \beta}^{\lambda} \delta a_{\lambda \sigma},
$$


which yields after some tedious, but straight forward manipulations

$$
\delta S_{\alpha \beta \sigma}=\boldsymbol{a}_{\sigma} \cdot \delta \boldsymbol{r}_{\mid \alpha \beta}+\left[b_{\alpha \beta} \boldsymbol{n}+S_{\alpha \beta}^{\lambda} \boldsymbol{a}_{\lambda}\right] \cdot \delta \boldsymbol{a}_{\sigma} .
$$

Note that we can rewrite the energy equivalently in terms of the contravariant form $S_{\alpha \beta}^{\sigma}$, which can be rewritten as

$$
\delta S_{\alpha \beta}^{\sigma}=\boldsymbol{a}^{\sigma} \cdot \delta \boldsymbol{r}_{\mid \alpha \beta}+\left[b_{\alpha \beta} a^{\sigma \lambda} \boldsymbol{n}-S_{\alpha \beta}^{\lambda} \boldsymbol{a}^{\sigma}\right] \cdot \delta \boldsymbol{a}_{\lambda},
$$

see Steigmann [21] for details. The above redefinition of the variation of the different strain measures in terms of $\delta \boldsymbol{a}_{\sigma}=\delta \boldsymbol{r}_{, \sigma}$ and $\delta \boldsymbol{r}_{\mid \alpha \beta}=\delta \boldsymbol{r}_{, \alpha \beta}-\bar{\Gamma}_{\alpha \beta}^{\sigma} \delta \boldsymbol{r}_{, \sigma}$ allows us now to write the internal virtual work as

$$
\delta \Psi=\boldsymbol{N}^{\alpha} \cdot \delta \boldsymbol{r}_{, \alpha}+\boldsymbol{M}^{\alpha \beta} \cdot \delta \boldsymbol{r}_{\mid \alpha \beta} .
$$

Comparing (32) and (34) with (37), (44) and (46) yields

$$
\begin{aligned}
\boldsymbol{N}^{\alpha}=\left[2\left(\frac{\partial \Psi}{\partial a_{\alpha \beta}}\right)^{\operatorname{sym}_{(\alpha \beta)}}+\right. & \left.\left(\frac{\partial \Psi}{\partial S_{\gamma \lambda \alpha}}\right)^{\operatorname{sym}_{(\gamma \lambda)}} S_{\gamma \lambda}^{\beta}\right] \boldsymbol{a}_{\beta}+ \\
& {\left[\left(\frac{\partial \Psi}{\partial S_{\gamma \lambda \alpha}}\right)^{\operatorname{sym}_{(\gamma \lambda)}} b_{\gamma \lambda}+\left(\frac{\partial \Psi}{\partial \kappa_{\gamma \lambda}}\right)^{\operatorname{sym}_{(\gamma \lambda)}} S_{\gamma \lambda}^{\alpha}\right] \boldsymbol{n} }
\end{aligned}
$$

and

$$
\boldsymbol{M}^{\alpha \beta}=\left(\frac{\partial \Psi}{\partial S_{\alpha \beta \sigma}}\right)^{\operatorname{sym}_{(\alpha \beta)}} \boldsymbol{a}_{\sigma}-\left(\frac{\partial \Psi}{\partial \kappa_{\alpha \beta}}\right)^{\operatorname{sym}_{(\alpha \beta)}} \boldsymbol{n} .
$$

Here, the symmetric part of the derivative $(\bullet)^{\operatorname{sym}_{\alpha \beta}}$ is introduced, where the symmetry condition is applied with respect to $\alpha$ and $\beta$, i.e.

$$
\left(\frac{\partial \Psi}{\partial S_{\alpha \beta \sigma}}\right)^{\operatorname{sym}_{(\alpha \beta)}}=\frac{1}{2}\left(\frac{\partial \Psi}{\partial S_{\alpha \beta \sigma}}+\frac{\partial \Psi}{\partial S_{\beta \alpha \sigma}}\right) .
$$

Next, we resolve the second covariant derivative

$$
\boldsymbol{M}^{\alpha \beta} \cdot \delta \boldsymbol{r}_{\mid \alpha \beta}=\boldsymbol{M}^{\alpha \beta} \cdot \delta \boldsymbol{r}_{, \alpha \beta}-\boldsymbol{M}^{\gamma \lambda} \bar{\Gamma}_{\gamma \lambda}^{\alpha} \cdot \delta \boldsymbol{r}_{, \alpha},
$$

such that we obtain for the virtual internal work

$$
\delta \Psi=\tilde{\boldsymbol{N}}^{\alpha} \cdot \delta \boldsymbol{r}_{, \alpha}+\boldsymbol{M}^{\alpha \beta} \cdot \delta \boldsymbol{r}_{, \alpha \beta},
$$

where

$$
\tilde{\boldsymbol{N}}^{\alpha}=\boldsymbol{N}^{\alpha}-\boldsymbol{M}^{\gamma \lambda} \bar{\Gamma}_{\gamma \lambda}^{\alpha}
$$

Insertion in (34) and subsequent integration by parts yields

$$
\delta W^{\text {int }}=\int_{\Omega}\left[\left(\tilde{\boldsymbol{N}}^{\alpha}-\boldsymbol{M}_{, \beta}^{\alpha \beta}\right) \cdot \delta \boldsymbol{r}+\boldsymbol{M}^{\alpha \beta} \cdot \delta \boldsymbol{r}_{, \beta}\right]_{, \alpha}-\left[\tilde{\boldsymbol{N}}_{, \alpha}^{\alpha}-\boldsymbol{M}_{, \alpha \beta}^{\alpha \beta}\right] \cdot \delta \boldsymbol{r} \mathrm{d} A .
$$

The results of the divergence theorem applied on the first term can be decomposed in normal and tangential direction, i.e. normal and tangential to the edges of the shell

$$
\begin{aligned}
\int_{\partial \Omega}\left[\left(\tilde{\boldsymbol{N}}^{\alpha}-\boldsymbol{M}_{, \beta}^{\alpha \beta}\right) \cdot \delta \boldsymbol{r}+\boldsymbol{M}^{\alpha \beta} \cdot \delta \boldsymbol{r}_{, \beta}\right] v_{\alpha} \mathrm{d} S= \\
\quad \int_{\partial \Omega}\left(\tilde{\boldsymbol{N}}^{\alpha} \cdot \delta \boldsymbol{r}\right) v_{\alpha}-\left(\boldsymbol{M}_{, \beta}^{\alpha \beta} \cdot \delta \boldsymbol{r}\right) v_{\alpha}+\left(\boldsymbol{M}^{\alpha \beta} \cdot \delta \boldsymbol{r}_{, v}\right) v_{\alpha} v_{\beta}+\left(\boldsymbol{M}^{\alpha \beta} \cdot \delta \boldsymbol{r}_{, \tau}\right) v_{\alpha} \tau_{\beta} \mathrm{d} S
\end{aligned}
$$

where $\boldsymbol{v}=v_{\alpha} \boldsymbol{A}^{\alpha}$ is the rightward unit normal to $\partial \Omega$ and $\boldsymbol{\tau}=\tau_{\alpha} \boldsymbol{A}^{\alpha}$ the tangential unit vector. Moreover, $\delta \boldsymbol{r}_{, \nu}=v^{\alpha} \delta \boldsymbol{r}_{, \alpha}$ and $\delta \boldsymbol{r}_{, \tau}=\tau^{\alpha} \delta \boldsymbol{r}_{, \alpha}$ are the corresponding derivatives, decomposed in tangential and normal direction. A secondary integration by parts with subsequent application of the divergence theorem of the last term on the right hand side of (56) on the boundary $\partial \Omega$ of the shell yields

$$
\int_{\partial \Omega} \boldsymbol{M}^{\alpha \beta} v_{\alpha} \tau_{\beta} \cdot \delta \boldsymbol{r}_{, \tau} \mathrm{d} S=\sum_{\partial^{2} \Omega}\left[\boldsymbol{M}^{\alpha \beta} v_{\alpha} \tau_{\beta}\right]_{i} \cdot \delta \boldsymbol{r}_{i}-\int_{\partial \Omega}\left(\boldsymbol{M}^{\alpha \beta} v_{\alpha} \tau_{\beta}\right)^{\prime} \cdot \delta \boldsymbol{r} \mathrm{d} S,
$$


where $(\bullet)^{\prime}=\mathrm{d}(\bullet) / \mathrm{d} S$ and the square brackets refer to the corner $i$ at the boundary. Assuming that the principle of virtual work $0=\delta W^{\text {int }}-\delta W^{\text {ext }}$ is valid with respect to the corresponding functional spaces of admissible solution and test functions defined at the end of this Section, the external contribution can be formulated as

$$
\delta W^{\mathrm{ext}}=\int_{\Omega} \boldsymbol{g} \cdot \delta \boldsymbol{r} \mathrm{d} A+\int_{\Upsilon} \boldsymbol{t} \cdot \delta \boldsymbol{r}+\boldsymbol{\mu} \cdot \delta \boldsymbol{r}_{, v} \mathrm{~d} S+\sum_{i} \boldsymbol{f}_{i} \cdot \delta \boldsymbol{r}_{i} .
$$

where the edge $\Upsilon$ is assumed to be an open set on $\partial \Omega$ and the vertices $i \in \Xi=[1, \ldots, n]$ are defined on $\partial^{2} \Omega$. Moreover, $\boldsymbol{g}$ denotes a distributed load, e.g. gravitational load. Eventually, we obtain the strong form of the second gradient problem as

$$
\left(\tilde{\boldsymbol{N}}^{\alpha}-\boldsymbol{M}_{, \beta}^{\alpha \beta}\right)_{, \alpha}+\boldsymbol{g}=\mathbf{0} \text { on } \Omega,
$$

with boundary conditions at the edges

$$
\begin{aligned}
& \boldsymbol{r}=\tilde{\boldsymbol{r}} \text { on } \Upsilon_{r}, \\
& \tilde{\boldsymbol{N}}^{\alpha} v_{\alpha}-\boldsymbol{M}_{, \beta}^{\alpha \beta} v_{\alpha}-\left(\boldsymbol{M}^{\alpha \beta} v_{\alpha} \tau_{\beta}\right)^{\prime}=\boldsymbol{t} \text { on } \Upsilon_{t} \text {, } \\
& \boldsymbol{r}_{, v}=\tilde{\boldsymbol{k}} \text { on } \Upsilon_{k}, \\
& \boldsymbol{M}^{\alpha \beta} v_{\alpha} v_{\beta}=\boldsymbol{\mu} \text { on } \Upsilon_{\mu}
\end{aligned}
$$

and at the vertices

$$
\begin{aligned}
\boldsymbol{r}_{i} & =\tilde{\boldsymbol{r}}_{i} \quad \text { on } \quad \Xi_{d}, \\
{\left[\boldsymbol{M}^{\alpha \beta} v_{\alpha} \tau_{\beta}\right]_{i} } & =\boldsymbol{f}_{i} \quad \text { on } \quad \Xi_{f}
\end{aligned}
$$

of the shell. As usual for fourth-order boundary value problems, we decompose the whole boundary twice. First, $\Upsilon=\Upsilon_{r} \cup \Upsilon_{t}$, with respect to $\Upsilon_{r} \cap \Upsilon_{t}=\emptyset$, and second, $\Upsilon=\Upsilon_{k} \cup \Upsilon_{\mu}$, with respect to $\Upsilon_{k} \cap \Upsilon_{\mu}=\emptyset$. Note that $\Upsilon_{r, t, k, \mu}$ are open sets on $\partial \Omega$, and we have additionally $\Xi=\Xi_{f} \cup \Xi_{d}$ with respect to $\Xi_{f} \cap \Xi_{d}=\emptyset$.

Eventually, we introduce the functional space of admissible solutions

$$
\mathcal{S}=\left\{\boldsymbol{r} \in \mathcal{H}^{2}(\Omega) \mid \boldsymbol{r}=\tilde{\boldsymbol{r}} \text { on } \Upsilon_{r}, \boldsymbol{r}_{, v}=\tilde{\boldsymbol{k}} \text { on } \Upsilon_{k}, \boldsymbol{r}_{i}=\tilde{\boldsymbol{r}}_{i} \text { on } \Xi_{d}\right\}
$$

and the space of admissible trial or test functions

$$
\mathcal{V}=\left\{\delta \boldsymbol{r} \in \mathcal{H}^{2}(\Omega) \mid \delta \boldsymbol{r}=\mathbf{0} \text { on } \Upsilon_{r}, \delta \boldsymbol{r}_{, v}=\mathbf{0} \text { on } \Upsilon_{k}, \delta \boldsymbol{r}_{i}=\mathbf{0} \text { on } \Xi_{d}\right\},
$$

required for the principle of virtual work. Note that the enforcement of the gradient terms in the space of admissible solutions is not trivial, see Schuß et al. [40] for details.

\section{Isogeometric discretization}

Concerning the spatial discretization, the surface domain $\Omega$ is subdivided into a finite set of non-overlapping elements $e \in \mathbb{N}$ such that

$$
\Omega \approx \Omega^{\mathrm{h}}=\bigcup_{e \in \mathbb{N}} \Omega_{e}
$$

Due to the use of the curvature coefficients in the presented model, the variational problem requires approximation functions which are globally at least $C^{1}$-continuous. To meet this continuity requirement an isogeometric analysis approach which employs Non-Uniform rational B-splines (NURBS) of order $p_{\alpha} \geq 2$ can be applied. Therefore, polynomial approximations of the deformed geometry in the shell mid-surface geometry $\boldsymbol{r}$ and its variation $\delta \boldsymbol{r}$ are defined as

$$
\boldsymbol{r}^{\mathrm{h}}=\sum_{I \in \mathcal{I}} R^{I} \boldsymbol{q}_{I} \quad \text { and } \quad \delta \boldsymbol{r}^{\mathrm{h}}=\sum_{J \in \mathcal{I}} R^{J} \delta \boldsymbol{q}_{J}
$$

respectively, where $\boldsymbol{q}_{I} \in \mathbb{R}^{3}$ and $\delta \boldsymbol{q}_{J} \in \mathbb{R}^{3}$. Introducing global shape functions $R^{I}: \Omega^{\mathrm{h}} \rightarrow \mathbb{R}$ associated with control points $I \in \mathcal{I}=\{1, \ldots, \mathfrak{n}\}$, NURBS based shape functions read

$$
R^{I}:=R^{i}(\boldsymbol{\theta})=\frac{\prod_{\alpha=1}^{2} B^{i_{\alpha}}\left(\theta^{\alpha}\right) w_{i}}{\sum_{j} \prod_{\alpha=1}^{2} B^{j_{\alpha}}\left(\theta^{\alpha}\right) w_{j}},
$$


where $B^{i_{\alpha}}$ are univariate non-rational B-splines defined on a parametric domain which is subdivided by the knot vector $\left[\theta_{1}^{\alpha}, \theta_{2}^{\alpha}, \ldots, \theta_{\mathfrak{n}_{\alpha}+p_{\alpha}+1}^{\alpha}\right], \mathfrak{n}=\mathfrak{n}_{1} \mathfrak{n}_{2}$. The recursive definition of a single univariate B-spline is given as follows

$$
B_{p_{\alpha}}^{i_{\alpha}}\left(\theta^{\alpha}\right)=\frac{\theta^{\alpha}-\theta_{i_{\alpha}}^{\alpha}}{\theta_{i_{\alpha}+p_{\alpha}}^{\alpha}-\theta_{i_{\alpha}}^{\alpha}} B_{p_{\alpha}-1}^{i_{\alpha}}\left(\theta^{\alpha}\right)+\frac{\theta_{i_{\alpha}+p_{\alpha}+1}^{\alpha}-\theta^{\alpha}}{\theta_{i_{\alpha}+p_{\alpha}+1}^{\alpha}-\theta_{i_{\alpha}+1}^{\alpha}} B_{p_{\alpha}-1}^{i_{\alpha}+1}\left(\theta^{\alpha}\right),
$$

beginning with

$$
B_{0}^{i_{\alpha}}\left(\theta^{\alpha}\right)= \begin{cases}1 & \text { if } \theta_{i_{\alpha}} \leq \theta^{\alpha}<\theta_{i_{\alpha}+1}^{\alpha} \\ 0 & \text { otherwise }\end{cases}
$$

Moreover, $w_{j}$ are corresponding NURBS weights. For further details on the construction of NURBS based shape functions as well as the construction of local refinements related to the IGA concept, see e.g. Cottrell et al. [34], Bornemann and Cirak [41], Hesch et al. [42] and Dittmann [43].

The approximations of the tangent vectors and their respective variations read

$$
\boldsymbol{a}_{\alpha}^{\mathrm{h}}:=\boldsymbol{r}_{, \alpha}^{\mathrm{h}}=\sum_{I \in \mathcal{I}} R_{, \alpha}^{I} \boldsymbol{q}_{I} \quad \text { and } \quad \delta \boldsymbol{a}_{\alpha}^{\mathrm{h}}:=\delta \boldsymbol{r}_{, \alpha}^{\mathrm{h}}=\sum_{J \in \mathcal{J}} R_{, \alpha}^{J} \delta \boldsymbol{q}_{J} .
$$

Thus, the approximations of the normal vector and its variation are given as

$$
\boldsymbol{n}^{\mathrm{h}}:=\frac{\boldsymbol{a}_{1}^{\mathrm{h}} \times \boldsymbol{a}_{2}^{\mathrm{h}}}{\left\|\boldsymbol{a}_{1}^{\mathrm{h}} \times \boldsymbol{a}_{2}^{\mathrm{h}}\right\|} \quad \text { and } \quad \delta \boldsymbol{n}^{\mathrm{h}}:=\frac{\left(\boldsymbol{I}-\boldsymbol{n}^{\mathrm{h}} \otimes \boldsymbol{n}^{\mathrm{h}}\right)\left(\boldsymbol{a}_{1}^{\mathrm{h}} \times \delta \boldsymbol{a}_{2}^{\mathrm{h}}+\delta \boldsymbol{a}_{1}^{\mathrm{h}} \times \boldsymbol{a}_{2}^{\mathrm{h}}\right)}{\left\|\boldsymbol{a}_{1}^{\mathrm{h}} \times \boldsymbol{a}_{2}^{\mathrm{h}}\right\|} .
$$

The approximations of the second covariant derivative and its variation read

$$
\boldsymbol{a}_{\alpha, \beta}^{\mathrm{h}}:=\boldsymbol{r}_{, \alpha \beta}^{\mathrm{h}}=\sum_{I \in \mathcal{I}} R_{, \alpha \beta}^{I} \boldsymbol{q}_{I} \quad \text { and } \quad \delta \boldsymbol{a}_{\alpha, \beta}^{\mathrm{h}}:=\delta \boldsymbol{r}_{, \alpha \beta}^{\mathrm{h}}=\sum_{J \in \mathcal{J}} R_{, \alpha \beta}^{J} \delta \boldsymbol{q}_{J}
$$

Moreover, the membrane strains, the in- and out-of-plane curvature as well as their variations are discretized as

$$
\begin{aligned}
& a_{\alpha \beta}^{\mathrm{h}}=\boldsymbol{a}_{\alpha}^{\mathrm{h}} \cdot \boldsymbol{a}_{\beta}^{\mathrm{h}} \text { and } \delta a_{\alpha \beta}^{\mathrm{h}}=\boldsymbol{a}_{\alpha}^{\mathrm{h}} \cdot \delta \boldsymbol{a}_{\beta}^{\mathrm{h}}+\boldsymbol{a}_{\beta}^{\mathrm{h}} \cdot \delta \boldsymbol{a}_{\alpha}^{\mathrm{h}}, \\
& \kappa_{\alpha \beta}^{\mathrm{h}}=\boldsymbol{a}_{\alpha, \beta}^{\mathrm{h}} \cdot \boldsymbol{n}^{\mathrm{h}} \quad \text { and } \delta \kappa_{\alpha \beta}^{\mathrm{h}}=\delta \boldsymbol{a}_{\alpha, \beta}^{\mathrm{h}} \cdot \boldsymbol{n}^{\mathrm{h}}+\boldsymbol{a}_{\alpha, \beta}^{\mathrm{h}} \cdot \delta \boldsymbol{n}^{\mathrm{h}}, \\
& S_{\alpha \beta \sigma}^{\mathrm{h}}=\boldsymbol{a}_{\alpha, \beta}^{\mathrm{h}} \cdot \boldsymbol{a}_{\sigma}^{\mathrm{h}}-\bar{\Gamma}_{\alpha \beta}^{\mathrm{h}, \lambda} a_{\sigma \lambda}^{\mathrm{h}} \quad \text { and } \delta S_{\alpha \beta \sigma}^{\mathrm{h}}=\delta \boldsymbol{a}_{\alpha, \beta}^{\mathrm{h}} \cdot \boldsymbol{a}_{\sigma}^{\mathrm{h}}+\boldsymbol{a}_{\alpha, \beta}^{\mathrm{h}} \cdot \delta \boldsymbol{a}_{\sigma}^{\mathrm{h}}-\bar{\Gamma}_{\alpha \beta}^{\mathrm{h}, \lambda} \delta a_{\sigma \lambda}^{\mathrm{h}} .
\end{aligned}
$$

To complete the approximation of the strain measures, the discrete right Cauchy-Green tensor and its variation is given as follows

$$
\hat{C}_{\alpha \beta}^{\mathrm{h}}=a_{\alpha \beta}^{\mathrm{h}}-2 \theta^{3} \boldsymbol{a}_{\alpha, \beta}^{\mathrm{h}} \cdot \boldsymbol{n}^{\mathrm{h}} \quad \text { and } \quad \delta \hat{C}_{\alpha \beta}^{\mathrm{h}}=\delta a_{\alpha \beta}^{\mathrm{h}}+2 \theta^{3} \delta \kappa_{\alpha, \beta}^{\mathrm{h}} .
$$

Now, using above approximations the discrete version of the internal virtual work given in (34) reads

$$
\delta W^{\mathrm{int}, \mathrm{h}}=\int_{\Omega^{\mathrm{h}}}\left[\left(n_{\mathrm{iso}, \mathrm{h}}^{\alpha \beta}+n_{\mathrm{fib}, \mathrm{h}}^{\alpha \beta}\right) \delta a_{\alpha \beta}^{\mathrm{h}}+\left(m_{\mathrm{iso}, \mathrm{h}}^{\alpha \beta}+m_{\mathrm{fib}, \mathrm{h}}^{\alpha \beta}\right) \delta \kappa_{\alpha \beta}^{\mathrm{h}}+m_{\mathrm{fib}, \mathrm{h}}^{\alpha \beta \sigma} \delta S_{\alpha \beta \sigma}^{\mathrm{h}}\right] \mathrm{d} A,
$$

where discrete versions of the stress resultants for the matrix material are given as

$$
n_{\text {iso,h }}^{\alpha \beta}=\int_{\varpi_{\text {iso }}} \frac{\partial \Psi^{\mathrm{iso}, \mathrm{h}}\left(\hat{C}_{i j}^{\mathrm{h}}\right)}{\partial \hat{C}_{\alpha \beta}^{\mathrm{h}}} \mathrm{d} \theta^{3} \quad \text { and } \quad m_{\text {iso,h }}^{\alpha \beta}=\int_{\varpi_{\text {iso }}} 2 \theta^{3} \frac{\partial \Psi^{\mathrm{iso}, \mathrm{h}}\left(\hat{C}_{i j}^{\mathrm{h}}\right)}{\partial \hat{C}_{\alpha \beta}^{\mathrm{h}}} \mathrm{d} \theta^{3}
$$

and for the fiber material as

$$
\begin{aligned}
n_{\mathrm{fib}, \mathrm{h}}^{\alpha \beta} & =\frac{\partial \Psi^{\mathrm{fib}, \mathrm{h}}\left(a_{\alpha \beta}^{\mathrm{h}}, S_{\alpha \beta \sigma}^{\mathrm{h}}, \kappa_{\alpha \beta}^{\mathrm{h}}\right)}{\partial a_{\alpha \beta}^{\mathrm{h}}}, \\
m_{\mathrm{fib}, \mathrm{h}}^{\alpha \beta} & =\frac{\partial \Psi^{\mathrm{fib}, \mathrm{h}}\left(a_{\alpha \beta}^{\mathrm{h}}, S_{\alpha \beta \sigma}^{\mathrm{h}}, \kappa_{\alpha \beta}^{\mathrm{h}}\right)}{\partial \kappa_{\alpha \beta}^{\mathrm{h}}}, \\
m_{\mathrm{fib}, \mathrm{h}}^{\alpha \beta \sigma} & =\frac{\partial \Psi^{\mathrm{fib}, \mathrm{h}}\left(a_{\alpha \beta}^{\mathrm{h}}, S_{\alpha \beta \sigma}^{\mathrm{h}}, \kappa_{\alpha \beta}^{\mathrm{h}}\right)}{\partial S_{\alpha \beta \sigma}^{\mathrm{h}}} .
\end{aligned}
$$




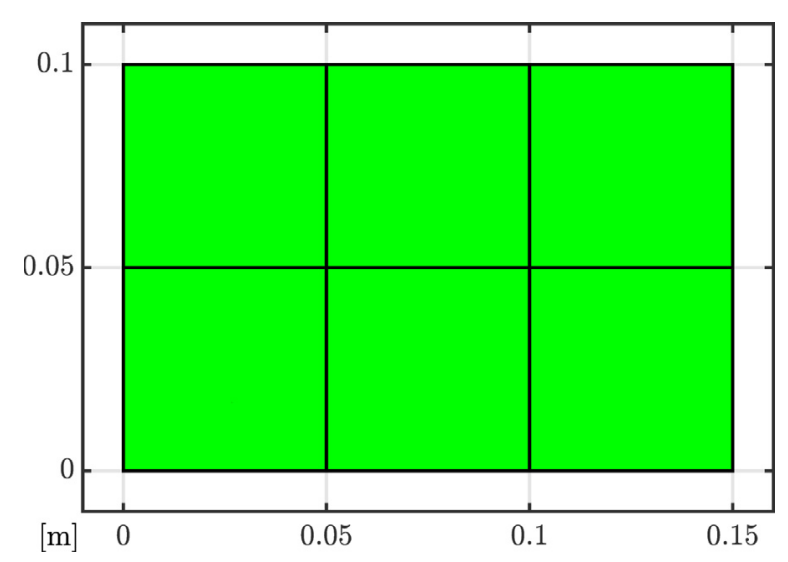

Fig. 2. Computational mesh consisting of $3 \times 2$ cubic B-spline based elements.

Moreover, the discrete version of the external virtual work given in (58) reads

$$
\delta W^{\mathrm{ext}, \mathrm{h}}=\int_{\Omega^{\mathrm{h}}} \boldsymbol{g} \cdot \delta \boldsymbol{r}^{\mathrm{h}} \mathrm{d} A+\int_{\Upsilon^{\mathrm{h}}} \boldsymbol{t} \cdot \delta \boldsymbol{r}^{\mathrm{h}}+\boldsymbol{\mu} \cdot\left(v^{\alpha, \mathrm{h}} \delta \boldsymbol{r}_{, \alpha}^{\mathrm{h}}\right) \mathrm{d} S+\sum_{i} \boldsymbol{f}_{i} \cdot \delta \boldsymbol{r}_{i}^{\mathrm{h}},
$$

where $\boldsymbol{v}^{\mathrm{h}}=v^{\alpha, \mathrm{h}} \boldsymbol{A}_{\alpha}$ is the discrete version of the rightward unit normal to $\partial \Omega^{\mathrm{h}}$.

\section{Numerical examples}

In the following, we investigate several numerical examples that assess the accuracy and performance of the proposed second gradient model. First, we investigate a series of benchmark examples with respect to analytical solutions. We make use of the incompressible Neo-Hookean model given in (41) as well as a compressible version given by

$$
\Psi^{\text {iso }}=\frac{1}{2} \mu\left(J^{-2 / 3} \operatorname{tr}(\hat{\boldsymbol{C}})-3\right)+\frac{1}{4} \kappa\left(J^{2}-1-2 \ln (J)\right)
$$

for the matrix material and the model given in (42) for the fiber material.

Secondly, we calibrate the combined matrix and fiber material with experimental measurements. Therefore, we make use of a compressible Neo-Hookean model for the matrix material given in (80).

Finally, a third example demonstrates the application of the model to a more complex, non-planar geometry using the fitted set of material parameters obtained within the previous examples. Here, we focus on the influence of the in-plane bending contributions of the fiber material.

\subsection{Verification}

In this section a step by step verification of the numerical framework is presented. Therefore, we conduct several numerical tests which allow us to investigate each effect of the fiber material separately. Unless otherwise defined, a shell of size $l \times b=0.15[\mathrm{~m}] \times 0.1[\mathrm{~m}]$ discretized by $3 \times 2$ cubic B-spline based elements is applied. The computational mesh and the material setting are given in Fig. 2 and Table 1, respectively. Note that for each Bspline based mesh considered in this section, knot vectors of the structure $\left[\theta_{1}^{\alpha}=\cdots=\theta_{p_{\alpha}+1}^{\alpha}<\cdots<\theta_{\mathfrak{n}_{\alpha}+1}^{\alpha}=\right.$ $\left.\cdots=\theta_{\mathfrak{n}_{\alpha}+p_{\alpha}+1}^{\alpha}\right]$ are applied.

\subsubsection{Tensile test}

We start our investigation with a tensile test to verify the behavior related to a stretching of the fibers, i.e. we set $a_{1}=a_{2}=h_{\mathrm{f}} E=12[\mathrm{kN} / \mathrm{m}]$ and neglect all other contributions of the fiber material. The left edge of a shell is fixed and the right edge is moved in $\boldsymbol{e}_{1}$-direction by $u$, see Fig. 3 for details.

In Fig. 4, the strain energy per unit reference area is depicted for the pure matrix material using a shell thickness of $h=0.002[\mathrm{~m}]$. Moreover, strain energy densities for the pure fiber material with $h_{\mathrm{f}}=0.002[\mathrm{~m}]$ as well 


\begin{tabular}{ll}
\hline Incompressible matrix material & \\
Shear modulus $\mu$ & $2[\mathrm{MPa}]$ \\
Thickness $h-h_{\mathrm{f}}$ & $0.001[\mathrm{~m}]$ \\
\hline Fiber material & \\
Young's modulus $E$ & $6[\mathrm{MPa}]$ \\
Thickness $h_{\mathrm{f}}$ & $0.001[\mathrm{~m}]$ \\
\hline
\end{tabular}

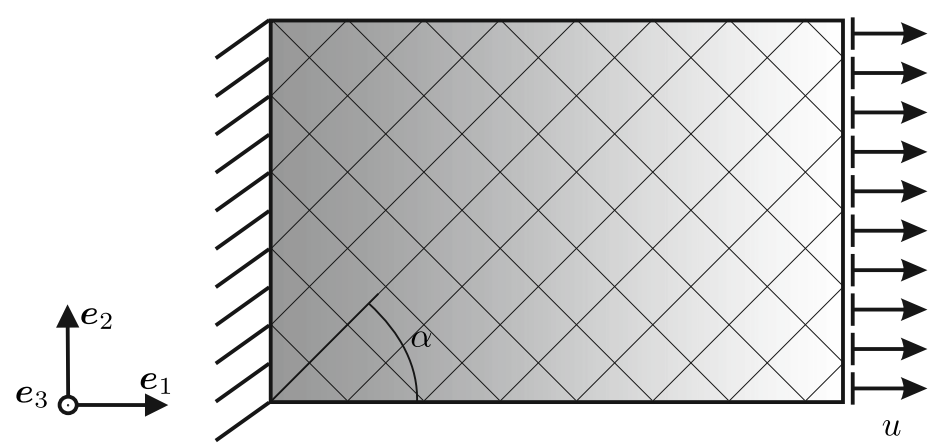

Fig. 3. Tensile test. Problem setting. The lines illustrate the fiber structure.

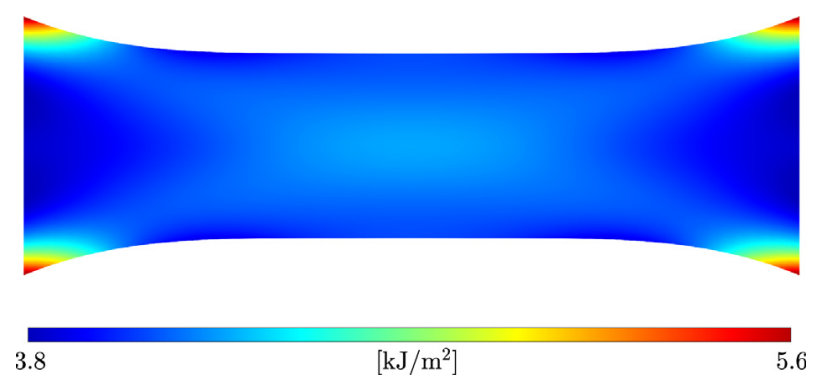

Fig. 4. Tensile test. Strain energy density of the matrix material at displacement $u=0.15[\mathrm{~m}]$.

as for the composite material using the setting from Table 1 are shown in Fig. 5. Therein fiber orientations of $\alpha=\left[0^{\circ}, 15^{\circ}, 30^{\circ}, 45^{\circ}\right]$ are applied. Note that the fiber material in the $0^{\circ}$ configuration does not experience lateral strains under longitudinal displacement conditions at the right edge. Here, we obtain a constant energy density of $6\left[\mathrm{~kJ} / \mathrm{m}^{2}\right]$ which is in accordance with the analytical solution, i.e. $\sum_{\alpha} a_{\alpha}\left(\lambda_{\alpha}-1\right)^{2} / 2$ with $\lambda_{1}=2$ and $\lambda_{2}=1$.

Fig. 6 shows the load deflection result, where results for the pure matrix material, the pure fiber material and the composite material are considered. Concerning the fiber material with a $0^{\circ}$ fiber orientation, we obtain a load of $1.2[\mathrm{kN}]$ which matches again with the analytical solution. This can be calculated in a straightforward manner using (49) and (60) by which we obtain a constant traction at the right boundary of $\boldsymbol{t}=\frac{a_{1} u}{l} \boldsymbol{e}_{1}$.

\subsubsection{Shear test}

Next, we deal with a simple shear test. To be specific, the lower edge of a shell is fixed, the left and right edges are fixed in $\boldsymbol{e}_{2}$-direction and the upper edge is moved in $\boldsymbol{e}_{1}$-direction by $u$ as illustrated in Fig. 7 . To verify the implementation, numerical results using pure fiber material $\left(h_{\mathrm{f}}=0.002[\mathrm{~m}]\right)$ have to match analytical solutions. Therefore, we set $\alpha=0^{\circ}, a_{1}=a_{2}=12[\mathrm{kN}]$ and $a_{3}=h_{\mathrm{f}} \mu=4[\mathrm{kN} / \mathrm{m}]$. All other parameters are set equal to zero.

Figs. 8 and 9 show the distribution of the strain energy density and the load deflection result, respectively. Therein, we obtain a homogeneous strain energy density of $3029.3\left[\mathrm{~J} / \mathrm{m}^{2}\right]$. The obtained results are in accordance to analytical solutions, see Appendix D for a detailed derivation. 

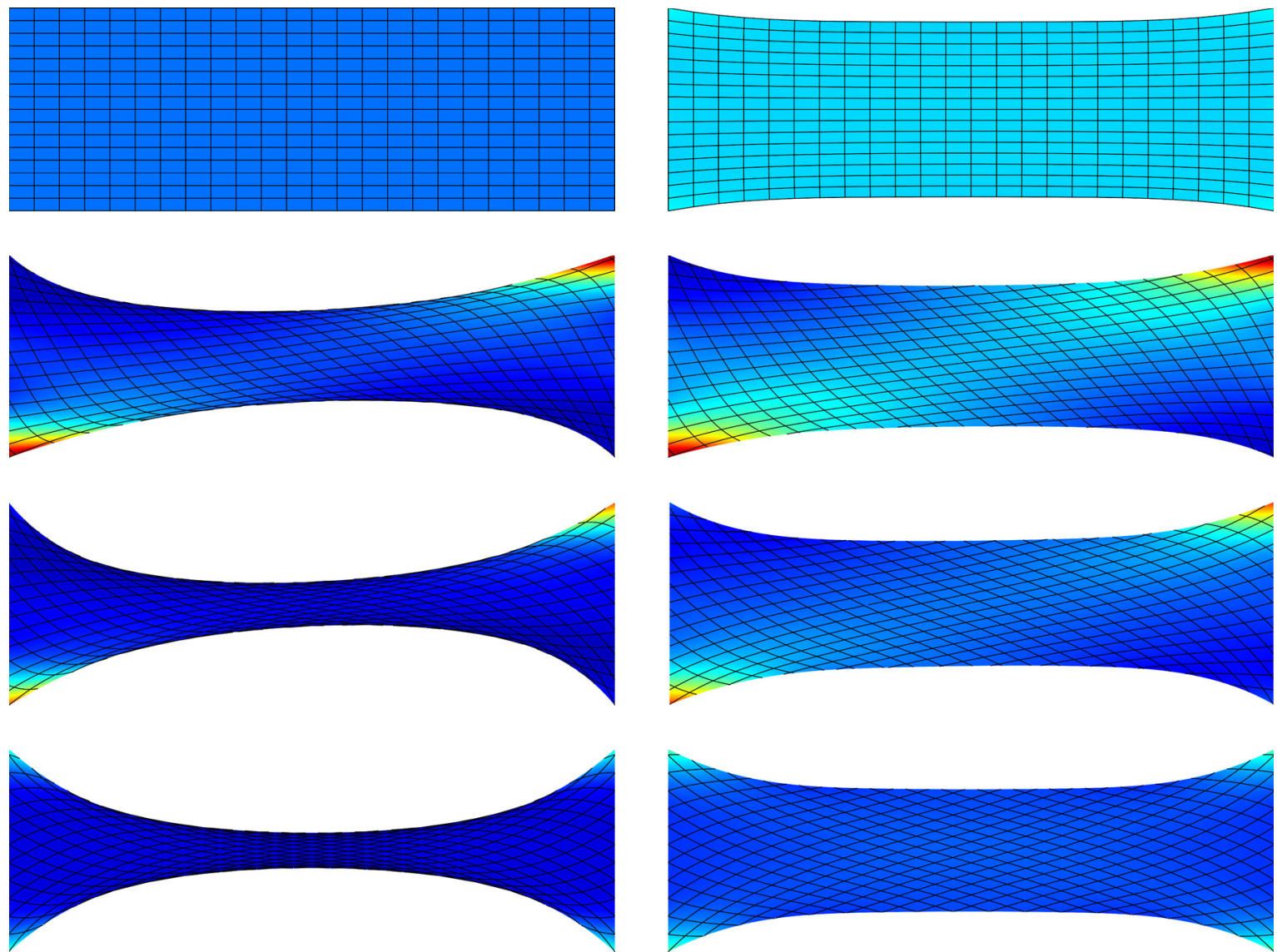

2

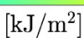

2

$\left[\mathrm{kJ} / \mathrm{m}^{2}\right]$

12

Fig. 5. Tensile test. Strain energy density of the fiber material (left) and the composite material (right) at displacement $u=0.15[\mathrm{~m}]$ and fiber configurations of $\alpha=\left[0^{\circ}, 15^{\circ}, 30^{\circ}, 45^{\circ}\right]$ (from top to bottom). The lines illustrate the deformed fiber structure.
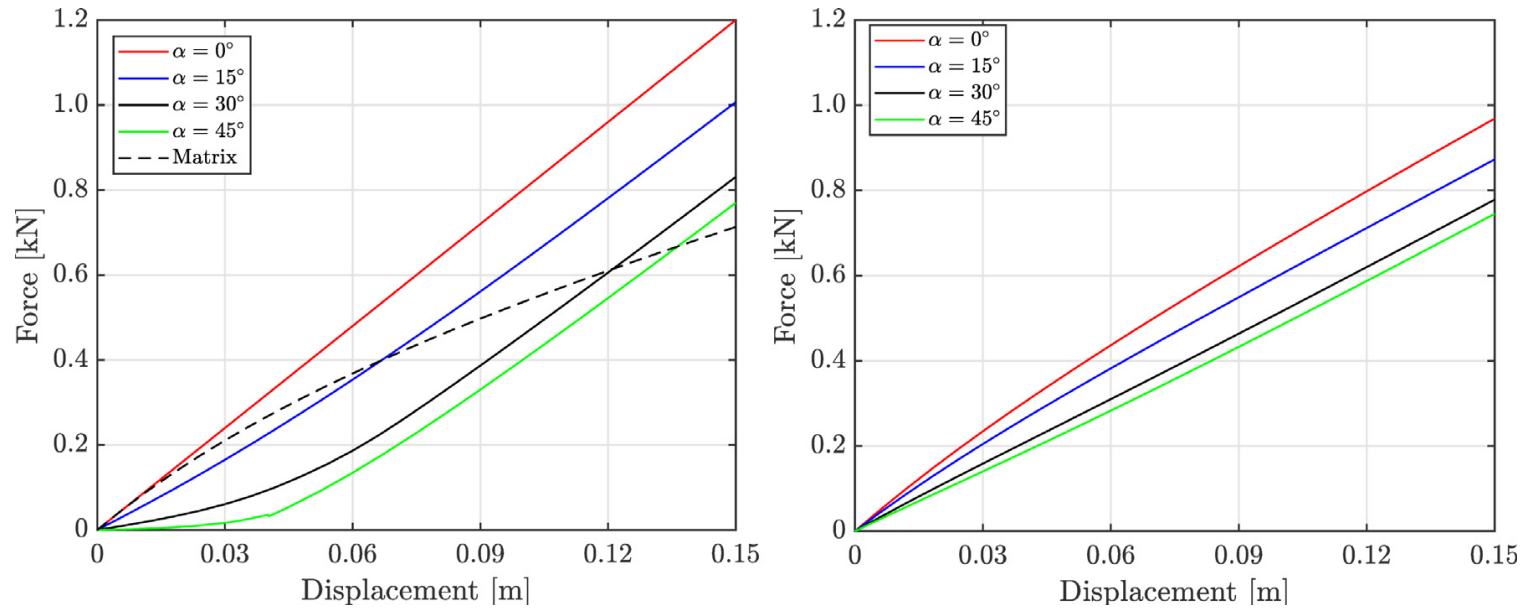

Fig. 6. Tensile test. Load deflection result of the fiber and matrix material (left) and the composite material (right). 


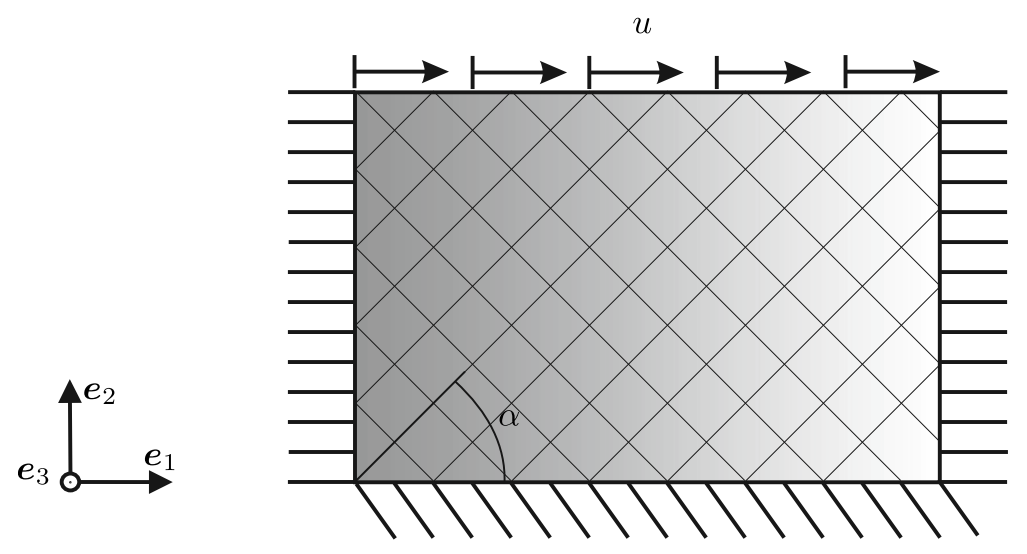

Fig. 7. Shear test. Problem setting. The lines illustrate the fiber structure.

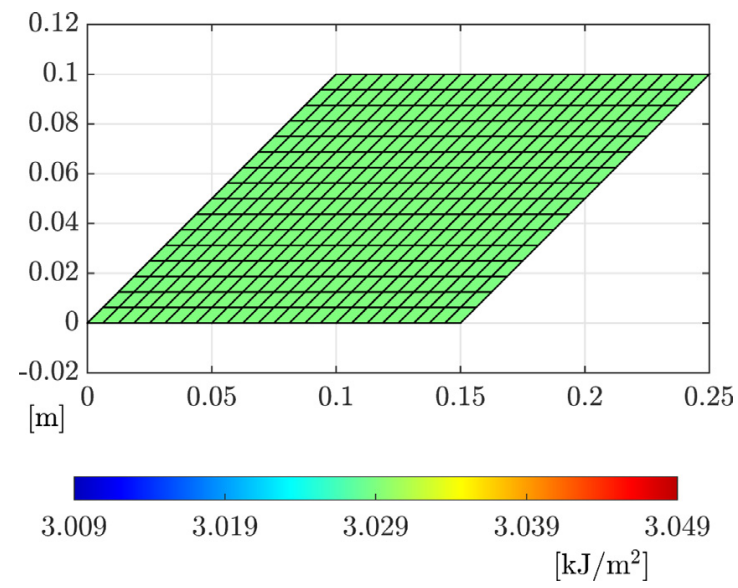

Fig. 8. Shear test. Strain energy density of the fiber material at displacement $u=0.1[\mathrm{~m}]$. The lines illustrate the deformed fiber structure.
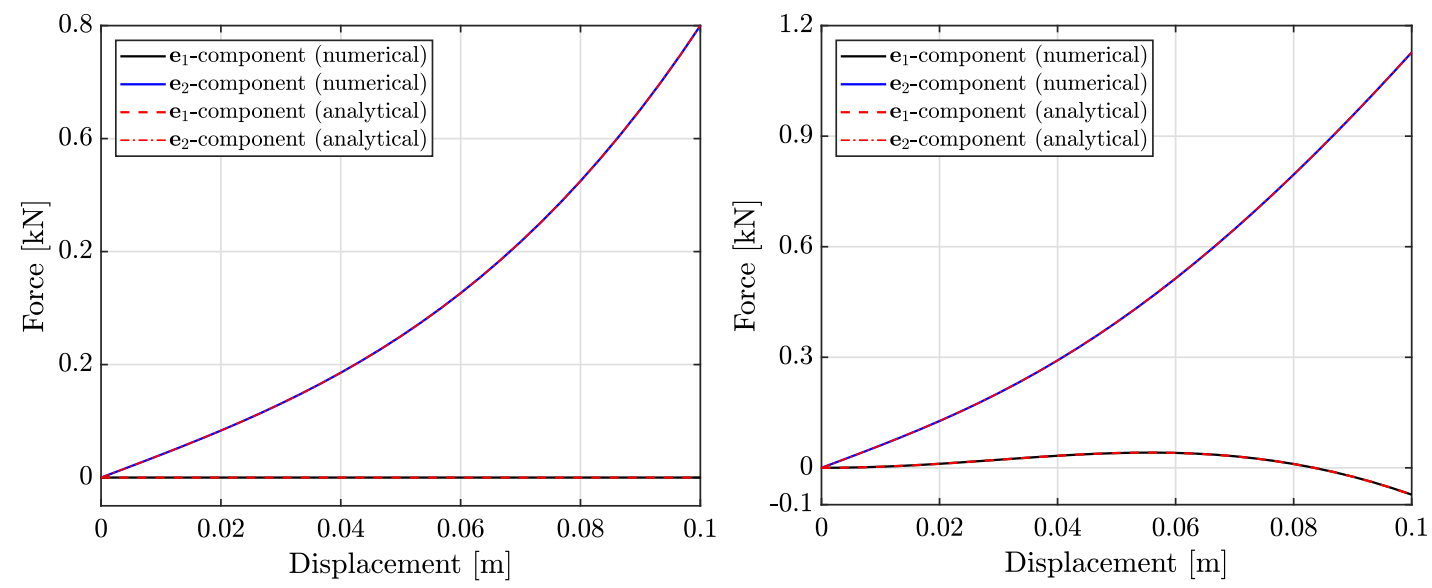

Fig. 9. Shear test. Load deflection result. Numerical and analytical solutions of resultant forces at the right edge $\Upsilon_{1}$ (left) and upper edge $\Upsilon_{2}$ (right) are depicted. 


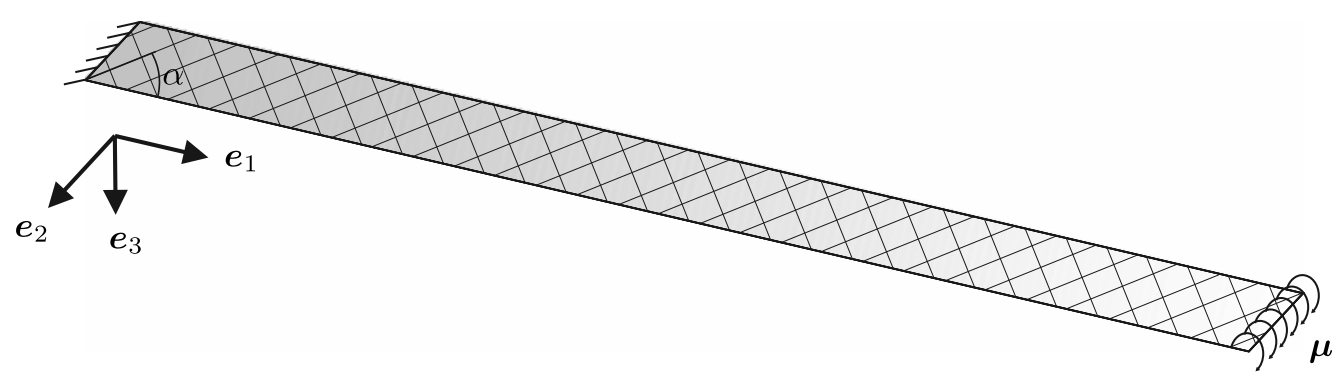

Fig. 10. Out-of-plane bending test. Problem setting. The lines illustrate the fiber structure.
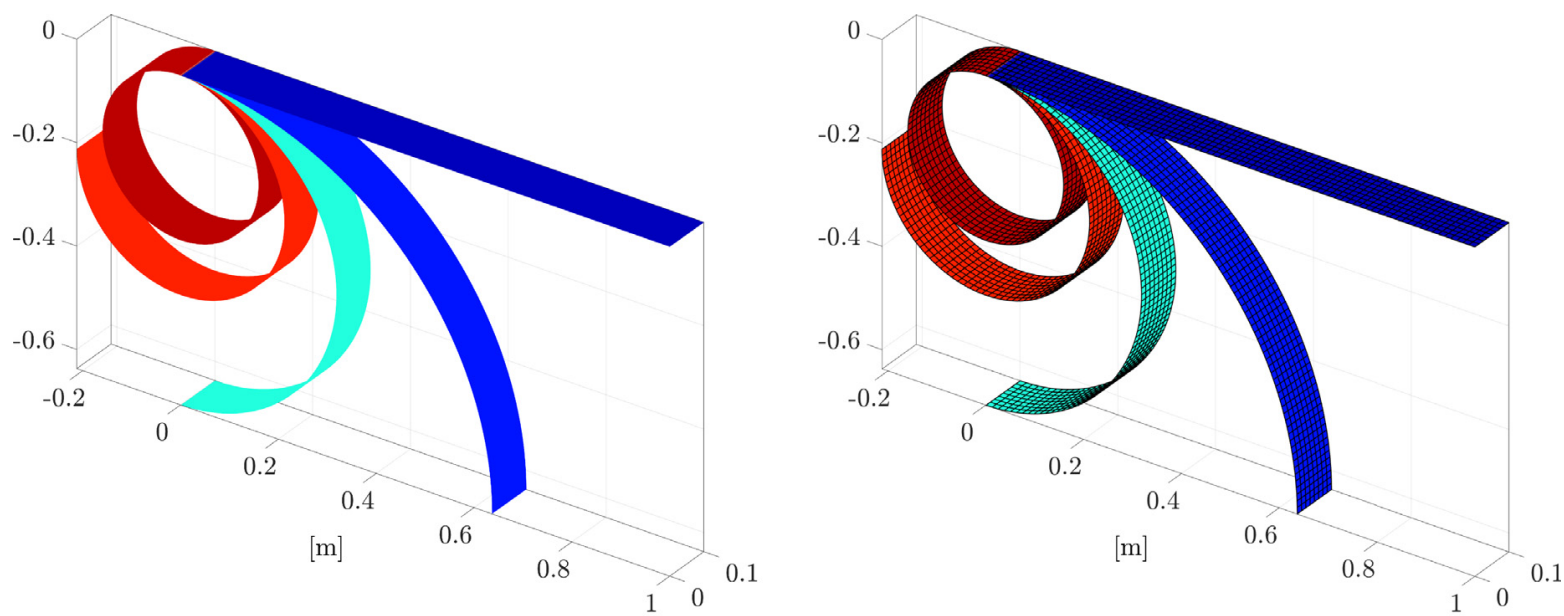

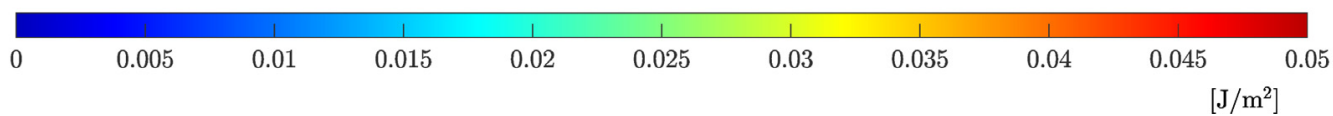

Fig. 11. Out-of-plane bending test. Configuration of the shell and strain energy distribution at different load steps. Pure matrix material (left) and pure fiber material (right) are applied. The lines illustrate the deformed fiber structure.

\subsubsection{Out-of-plane bending test}

For the out-of-plane bending test, the left edge of a shell is clamped and the right edge is subject to an external out-of-plane torque $\boldsymbol{\mu}=M \boldsymbol{e}_{2}$ as shown in Fig. 10. In contrast to previous tests, the shell used for this test has a length of $l=1[\mathrm{~m}]$ and a width of $b=0.1[\mathrm{~m}]$ in the reference configuration, see Fig. 11. Using a thickness of $h=0.002[\mathrm{~m}]$ for pure matrix material and a thickness of $h_{\mathrm{f}}=0.002[\mathrm{~m}]$ for pure fiber material, the area moment of inertia is obtained by $I=b h^{3} / 12=b h_{\mathrm{f}}^{3} / 12=66.6667\left[\mathrm{~mm}^{4}\right]$. The applied torque $M=2 \pi E I / l$ is chosen such that the shell describes a perfect circle in the asymptotic limit (see e.g. [36]) which holds for the fiber material by setting $\alpha=0^{\circ}, a_{1}=h_{\mathrm{f}} E=12[\mathrm{kN} / \mathrm{m}]$ and $k_{1}=E I / b=0.004[\mathrm{Nm}]$. Moreover, for the matrix material we apply the compressible model given in (80) with $\mu=E / 2=3[\mathrm{MPa}]$ and $\kappa=E / 3=2[\mathrm{MPa}$ ] which corresponds to Poisson ratio of $v=0$.

In Fig. 11, the configuration of the shell is plotted along with the strain energy distribution for pure matrix and pure fiber material at different load steps. The results shown therein are obtained by $80 \times 8$ cubic B-spline based elements. Moreover, Fig. 12 shows the convergence properties for both materials, where the error is determined as gap between the ends of the shell. Here, we observe a limit of convergence at a value of approximately $10^{-4}[\mathrm{~m}]$ for pure matrix material, whereas for pure fiber material an error of less than $2 \cdot 10^{-8}[\mathrm{~m}]$ is obtained. Note that the limit of convergence using pure matrix material can be justified by known locking effects of Kirchhoff-Love shell elements as it may occur even for high polynomial approximations, see e.g. [44-50]. In addition, the usage of an internal Newton-Raphson iteration within the implementation of a compressible Neo-Hookean model for the matrix material also limits the convergence. 


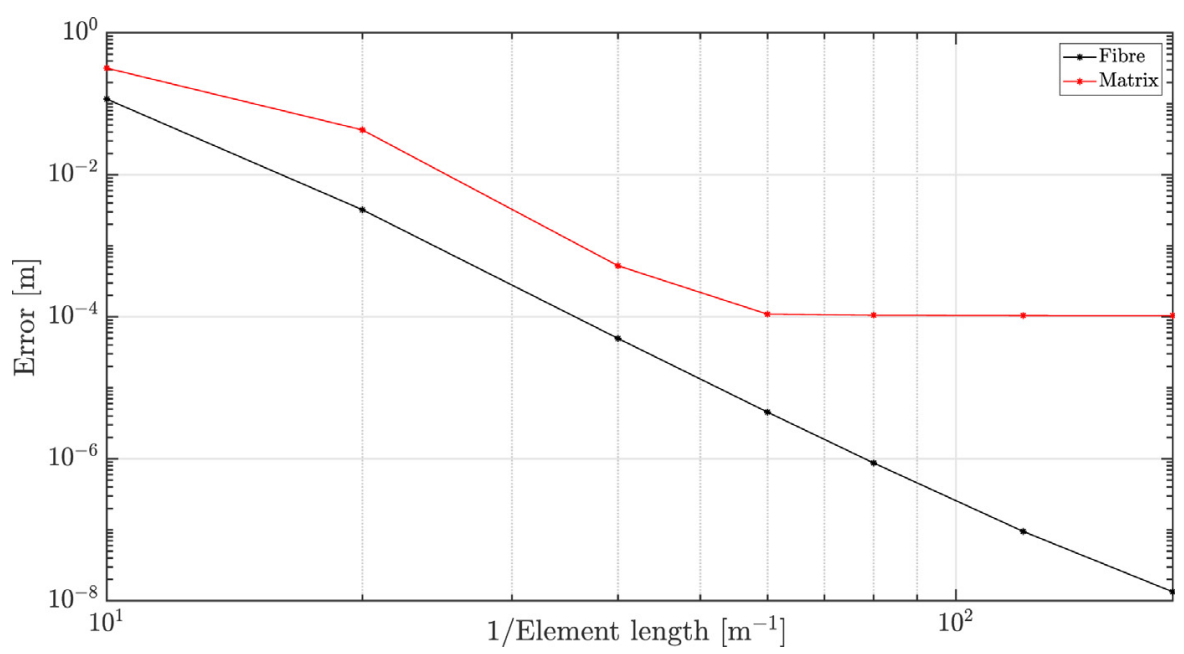

Fig. 12. Out-of-plane bending test. Convergence results.

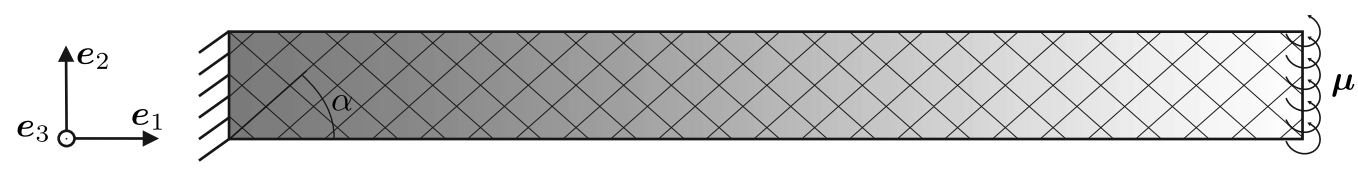

Fig. 13. In-plane bending test. Problem setting. The lines illustrate the fiber structure.

Next, a verification of the in-plane bending behavior of the fiber material is demonstrated. Therefore, we use the shell mesh defined for the out-of-plane bending test and set $\alpha=0^{\circ}, a_{1}=h_{\mathrm{f}} E=12[\mathrm{kN} / \mathrm{m}]$ with $h_{\mathrm{f}}=0.002[\mathrm{~m}]$ and $g_{1}=g_{2}=E \bar{I} / b=10[\mathrm{Nm}]$ with $\bar{I}=h_{\mathrm{f}} b^{3} / 12=166667\left[\mathrm{~mm}^{4}\right]$. All other parameters of the fiber material are set equal to zero. Moreover, we apply $\boldsymbol{\mu}=M \boldsymbol{e}_{3}$ as an external in-plane bending torque, see Fig. 13 for details. Again, the applied torque $M=2 \pi E \bar{I} / l$ is chosen such that the shell describes a perfect circle in the asymptotic limit, which is demonstrated in Fig. 14. Even if this result is not physically reasonable for a real composite due to the material penetration, it verifies the implementation related to in-plane bending of pure fiber material.

\subsubsection{In-plane bending test}

\subsubsection{Torsion test}

Eventually, we verify the numerical framework related to a torsional deformation of the fiber material, see Fig. 15. Therefore, the deformation is predetermined such that the shell undergoes a constant twist of $\partial \bar{\phi} / \partial X_{1}=1200[\% / \mathrm{m}]$ with $X_{1}=\boldsymbol{X} \cdot \boldsymbol{e}_{1}$. Moreover, the parameters of the fiber material are defined by $h_{\mathrm{f}}=0.002[\mathrm{~m}]$ and $k_{3}=\mu I_{\mathrm{p}} / b=$ $5.002[\mathrm{Nm}]$ with a polar moment of inertia $I_{\mathrm{p}}=b h_{\mathrm{f}}\left(b^{2}+h_{\mathrm{f}}^{2}\right) / 12=166733\left[\mathrm{~mm}^{4}\right]$. Again, all other parameters are set equal to zero.

Strain energy densities obtained at $\Delta \bar{\phi}=180^{\circ}$ are depicted in Fig. 16 for different fiber configurations. As expected, we obtain a highly stressed region along the central axis of the shell especially in the $0^{\circ}$ fiber configuration, whereas the fiber material does not contribute to the strain energy in the $45^{\circ}$ configuration since the fibers are not twisted. This is also evident in Fig. 17, where the twist of fiber is plotted for fiber configurations of $\alpha=0^{\circ}$ and $\alpha=45^{\circ}$. Concerning the $0^{\circ}$ configuration, fibers in $\boldsymbol{e}_{1}$-direction undergo a twist of $\partial \bar{\phi} / \partial X_{1}=1200[\% / \mathrm{m}]$ at the central axis of the shell. Moreover, analytical details related to the torsional test can be found in Appendix $\mathrm{E}$ verifying the numerical results shown in Fig. 16.

\subsection{Calibration and validation}

As introduced in Section 1.2, we investigate Tepex ${ }^{\circledR}$ dynalite 102-RG600(1)/47 from LANXESS as prototypical composite material, consisting of $47 \%$ vol. of a woven fabric (roving glass) and polycaprolactam (PA 6) as matrix 


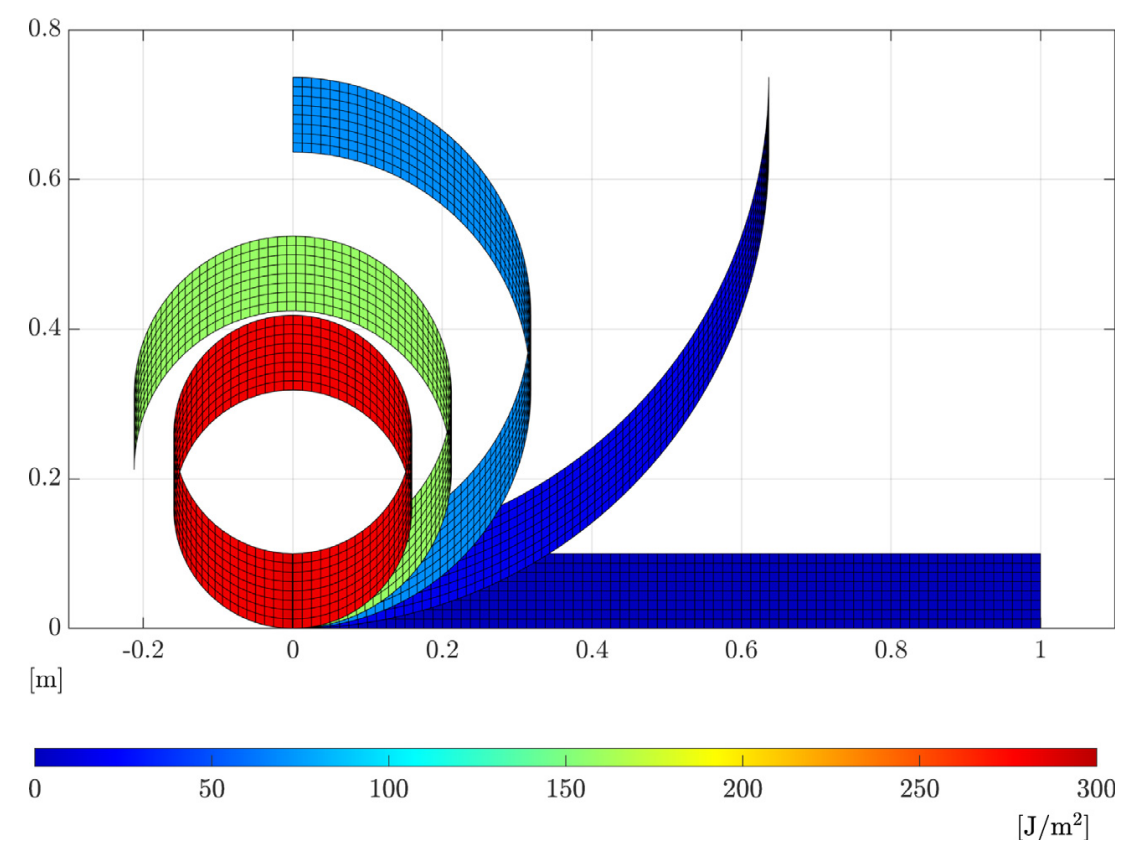

Fig. 14. In-plane bending test. Configuration of the shell and strain energy distribution at different load steps using pure fiber material. The lines illustrate the deformed fiber structure.

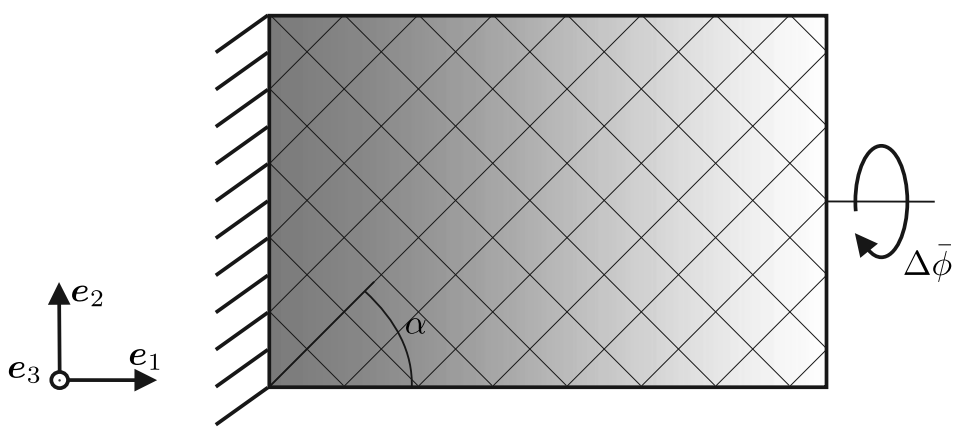

Fig. 15. Torsion test. Problem setting. The lines illustrate the fiber structure.

material. The matrix material is hydrophilic with a maximum absorption of moisture between $2.6 \%$ and $3.4 \%$. For the investigations presented here, a single layer material with layer thickness of $0.5[\mathrm{~mm}]$ has been specifically manufactured for this test, since multi-layered material with different fiber angles intermix various physical effects within the measurements.

The surrounding matrix material stiffens the woven fabric and connects the junctions of the fibers. The fibers within the composite material have significant bending stiffness due to the matrix material surrounding every single string within the fibers. Additional stiffening mechanisms are present depending on the actual weave of the woven fabric, since the fibers are subject to frictional behavior at the junctions. These effects arise only for the combined composite material and thus, cannot be separated within the experimental investigations, i.e. we always obtain a mean value of the material characteristics.

Remark 3. The matrix material is in general governed by a far more complex constitutive behavior in the inelastic regime. Moreover, fibers and matrix might separate in the presence of large deformations, i.e. both materials can be characterized by separately assigned deformation fields, coupled via corresponding constitutive laws. For now, we restrict ourself to the hyperelastic regime without fiber separation. 

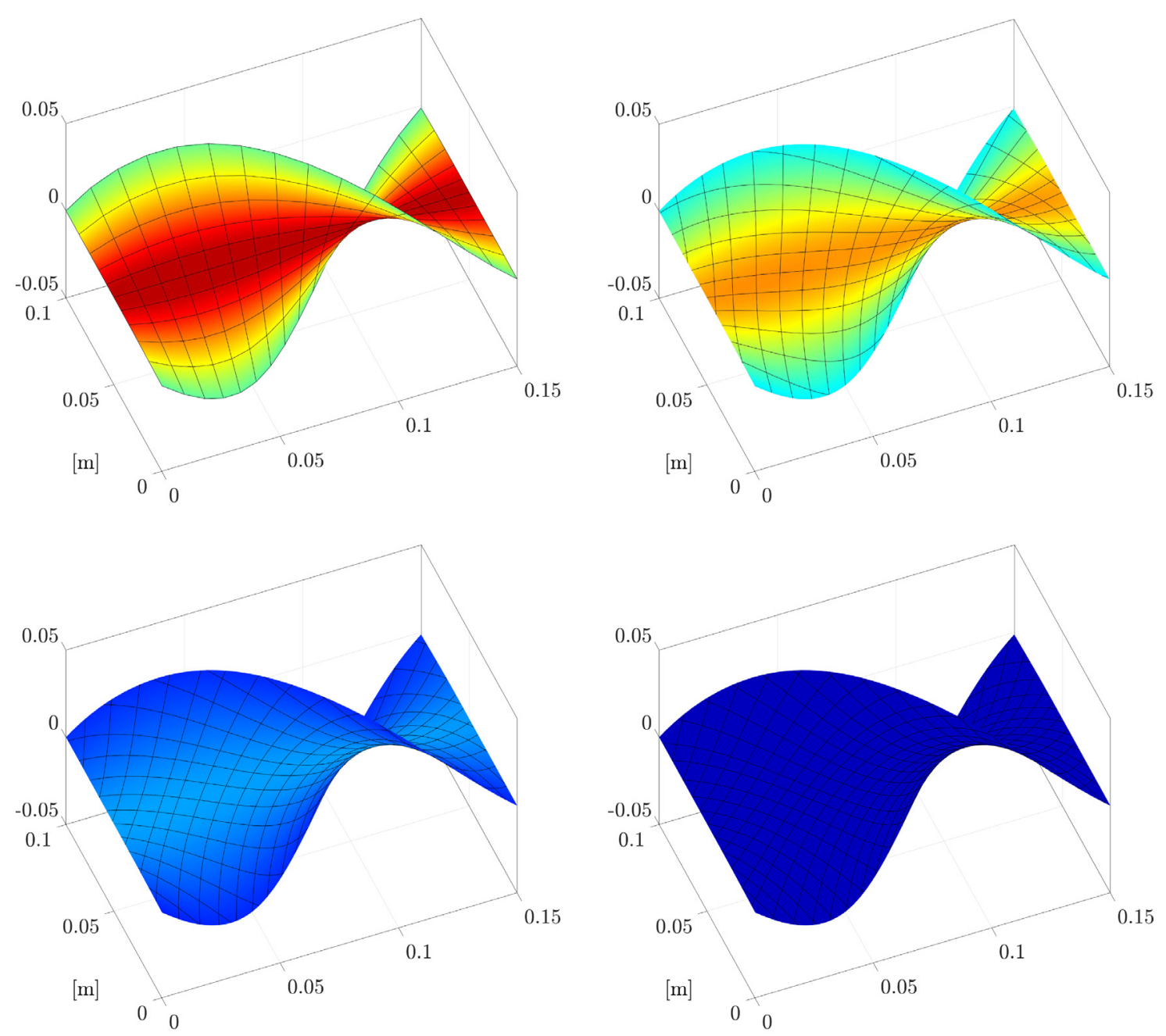

\begin{tabular}{|c|c|c|c|c|c|c|c|c|c|}
\hline & & t & 1 & $\perp$ & 1 & 1 & 1 & $\perp$ & 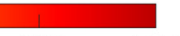 \\
\hline 100 & 200 & 300 & 400 & 500 & 600 & 700 & 800 & 900 & 1000 \\
\hline
\end{tabular}

Fig. 16. Torsion test. Strain energy distribution of the fiber material at $\Delta \bar{\phi}=180^{\circ}$ using different fiber configurations $\alpha=\left[0^{\circ}, 15^{\circ}, 30^{\circ}, 45^{\circ}\right]$ (from left to right and top to bottom). The lines illustrate the deformed fiber structure.

\subsubsection{Experimental setup}

For the tests on the fiber reinforced material, standard specimens with $125[\mathrm{~mm}] \times 25[\mathrm{~mm}]$ and $125[\mathrm{~mm}] \times$ $36[\mathrm{~mm}]$ were prepared and pulled with $0.5[\mathrm{~mm} / \mathrm{s}]$ on a universal testing machine. To prevent slipping between the testing machine and the specimen, considerable clamping forces are required. To preserve the underlying material from fracturing due to the clamping forces, additional 20 [mm] cap strips (width is adjusted to the sample) made from the same material with fiber orientation rotated $45^{\circ}$ against the orientation of the fibers of the samples were glued at both sides at the ends of the specimen. Fig. 18 displays the clamping device for a 36 [mm] specimen.

For the digital image correlation measurements of the fiber reinforced material, the optical system Q400 from Limess \& Software GmbH was used. The evaluation of the images was done in Instra4D, version 4.1. For the analysis, Instra4D generates a quadratic mesh within the region of interest with an element size of 5 pixels. At each node of the mesh, a facet of 29 pixels was applied, i.e. each facet is slightly larger compared to a single element of the correlation mesh. Note that at least one characteristic object of the speckle-pattern has to be inside every facet. 


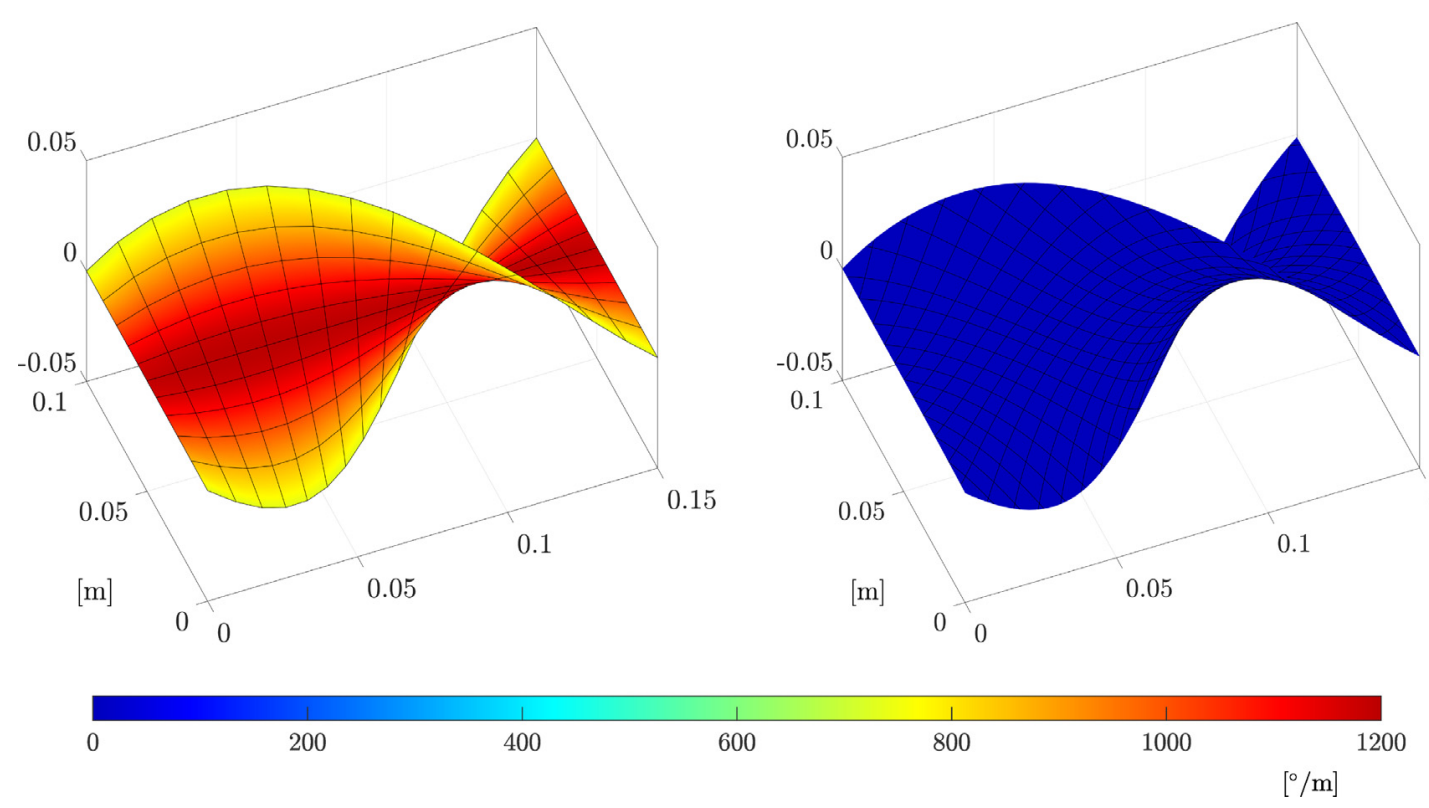

Fig. 17. Torsion test. Twist of fiber at $\Delta \bar{\phi}=180^{\circ}$ using a fiber configuration of $\alpha=0^{\circ}$ (left) and $45^{\circ}$ (right). The lines illustrate the deformed fiber structure.

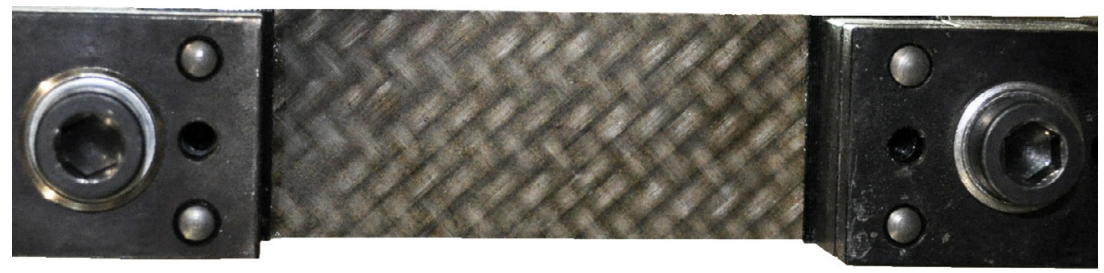

Fig. 18. Clamping devise with $36 \mathrm{~mm}$ specimen, undeformed configuration.

To control the calculated strains, the position of each identified object in the current configuration was calculated back into the reference configuration and compared with the original picture with a maximum tolerance of 0.3 pixel.

In Fig. 19, the measurements until failure of the $25[\mathrm{~mm}]$ specimen with $30^{\circ}$ fiber orientation are presented. As can be seen, a damage process starts after $0.4[\mathrm{~mm}]$ corresponding to a load of $500[\mathrm{~N}]$ and the fracture process begins at a displacement of approximately $6[\mathrm{~mm}]$. In particular, the fibers start to rupture as indicated by the post-fracture curves. Since we are interested in the elastic material behavior, we restrict the following diagrams to the regions of interest.

\subsubsection{Results}

Tension test. We first elaborated tension tests with $0^{\circ}, 15^{\circ}, 30^{\circ}$ and $45^{\circ}$ fiber orientation. Here, experimental data of 25 [mm] specimen are compared with numerical results using $34 \times 10$ cubic elements. Shear and bulk modulus used within the compressible model (80) for the PA 6 matrix material are assumed to be $\mu=384.62\left[\mathrm{~N} / \mathrm{mm}^{2}\right]$ and $\kappa=833.33\left[\mathrm{~N} / \mathrm{mm}^{2}\right]$, respectively. This setting corresponds to Young's modulus of $E_{\text {iso }}=1000\left[\mathrm{~N} / \mathrm{mm}^{2}\right]$ and Poisson ratio of $v=0.3$. Moreover, for the glass fibers we assume $E_{\mathrm{fib}}=73000\left[\mathrm{~N} / \mathrm{mm}^{2}\right]$. The thickness of the material is $h=0.5$ [mm], with $47 \%$ vol. of woven fabric, from which $50 \%$ are aligned in $L$ and $M$ direction, respectively. Thus, we obtain the material constants for the fiber stretch contributions via $0.5 h E_{\text {fib }} \times 0.47$ as $a_{1 / 2}=8577.5[\mathrm{~N} / \mathrm{mm}]$, which corresponds to a combined Young's modulus of $17685\left[\mathrm{~N} / \mathrm{mm}^{2}\right]$. As can be seen in Fig. 20 for the $0^{\circ}$ fiber orientation, the simulation fits perfectly the experiments.

The value for $a_{3}=250[\mathrm{~N} / \mathrm{mm}]$ is related to the interaction between the fibers and cannot be derived from other constants. Thus, we have fitted the value to the experimental observation obtained by a fiber orientation of $45^{\circ}$, 


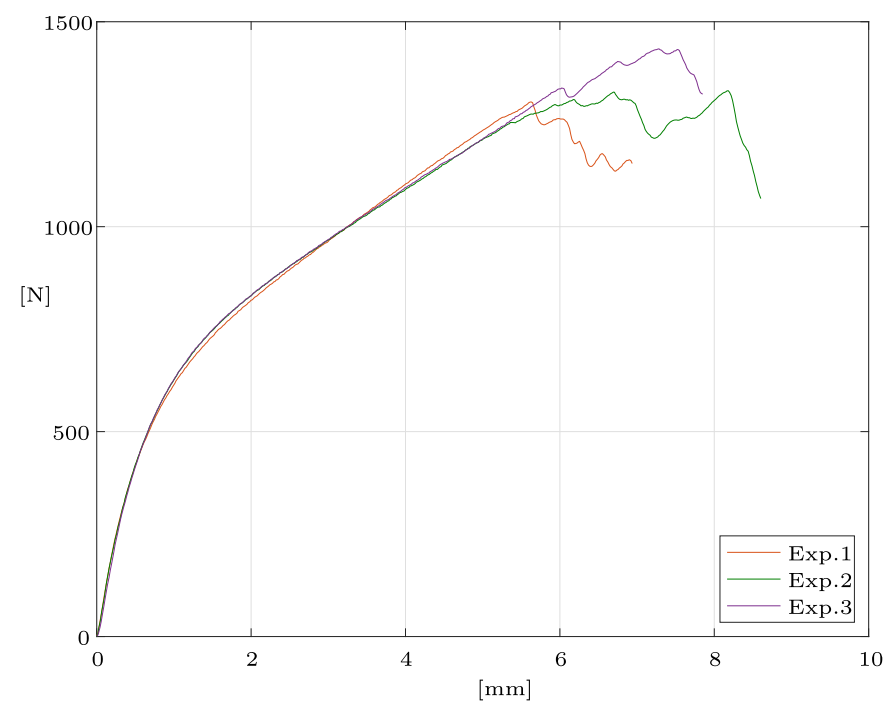

Fig. 19. Experimental force-displacement curves for $25 \mathrm{~mm}$ specimen with $30^{\circ}$ fiber orientation.
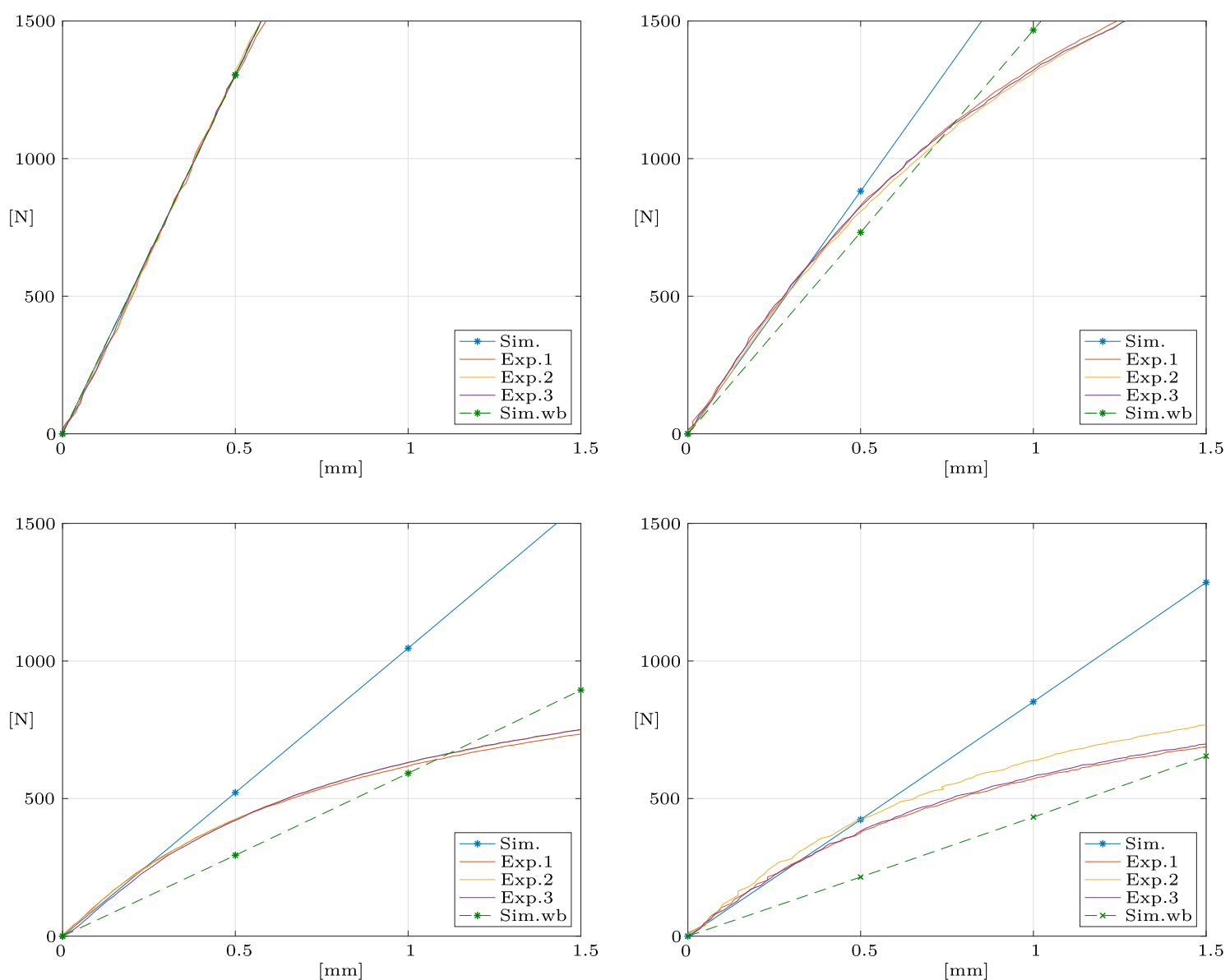

Fig. 20. Force-displacement curves of the tension test for $25 \mathrm{~mm}$ specimen with $0^{\circ}, 15^{\circ}, 30^{\circ}$ and $45^{\circ}$ fiber orientation (left to right, top to down). Simulation results are compared with experimental investigations. Additionally, simulation results without in-plane bending stiffness (Sim. wb) are shown to highlight the global effect of the non-classical in-plane curvature term. 


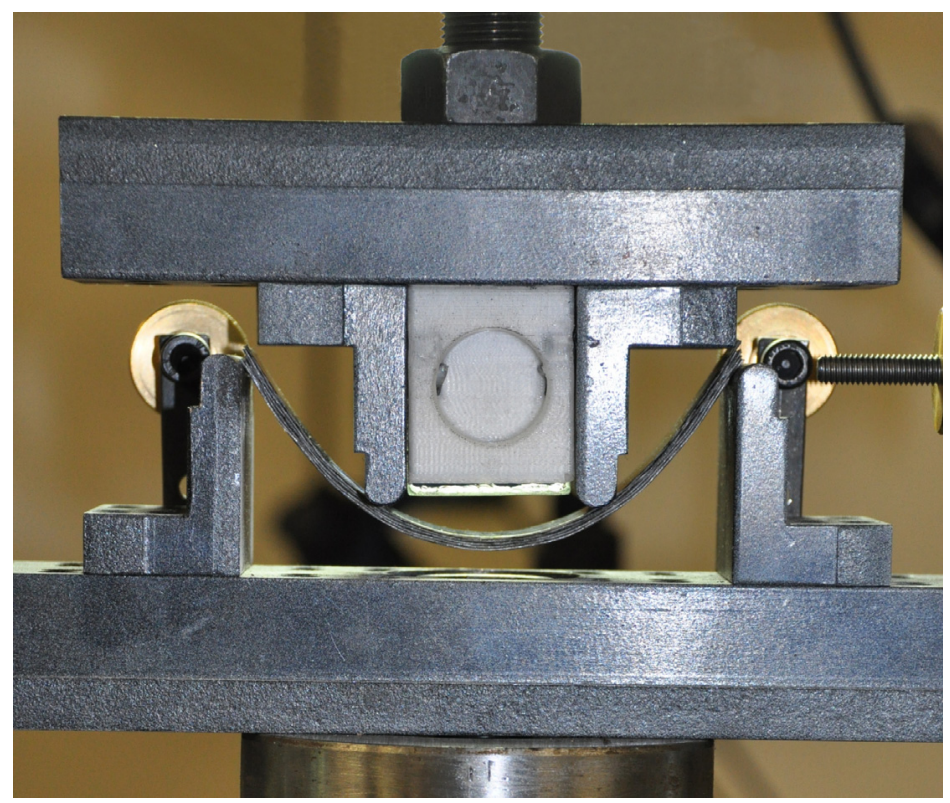

Fig. 21. 4-point bending test, deformed configuration.

see Fig. 20 (right, bottom). The bending stiffness $g_{1}=g_{2}$ on the other hand is related to Young's modulus via $g_{1 / 2}=E_{\mathrm{fib}} \bar{I} / b=893500[\mathrm{Nmm}]$, where the second moment of inertia is $\bar{I}=b^{3} h_{\mathrm{f}} / 12$ with $h_{\mathrm{f}}=0.47 h$. Note that this approach is only a motivation for choosing the material constants $g_{1}$ and $g_{2}$, since this allows us a comparison with analytical results as shown in Section 5.1.4. The values $k_{1 / 2 / 3}$ do not contribute in these tension tests and $g_{3}$ was set to zero. Additional simulation results presented in Fig. 20 demonstrate the effects of the in-plane bending term, i.e. we set $g_{1}=g_{2}=0$, to compare the results with an anisotropic Kirchhoff-Love shell formulation neglecting the higher gradient terms.

This example shows that the bending stiffness has considerable effects on the global stiffness and is not negligible. The constitutive parameters chosen with respect to the different matrix and fiber materials and the respective geometry allow for a nearly perfect prediction of the macroscopic behavior of this material with microstructure. Only the parameter $a_{3}$ is fitted, since the shear stiffness of the fibers depends on the weave of the fabric and the production process in terms of applied pressure, heat and cooling rate. This result illustrates the necessity to include higher gradient terms in the model for fiber reinforced polymers.

Bending test. Next, we investigate the out-of-plane bending stiffness, hence we validate the values for $k_{1}$ and $k_{2}$. Therefore, a 4-point bending test as shown in Fig. 21 is conducted. For the experiments, a stack of 5 specimen with $125[\mathrm{~mm}] \times 25[\mathrm{~mm}]$ in $0^{\circ}$ fiber configuration were used, such that we expect a stiffness of 5 times $k_{1 / 2}=E_{\mathrm{fib}} I / b=78.949$ [Nmm], where $I=b h_{\mathrm{f}}^{3} / 12$ and $h_{\mathrm{f}}=0.47 h$. The support structure has a width of 40 [mm] between the inner brackets, and $30[\mathrm{~mm}]$ between the outer and the inner brackets, such that the whole device has a width of 100 [mm].

For the numerical validation, we discretize the shell with $50 \times 10$ cubic elements with prescribed displacements in upward direction at the contact points of the support structure, such that the shell can slide over the bracket points and the system is subject to pure bending. The results in Fig. 22 demonstrate the consistency of the formulation with the experimental observations until a displacement of about 15 [mm]. Afterwards, inelastic effects can be observed, resulting in a permanent deformation of the sample as can be observed in Fig. 23. After a displacement of 25 [mm], the sample starts to slip over the brackets.

Digital image correlation. Finally, we show in Fig. 24 (top) the results of the Digital image correlation for the 25 [mm] specimen with $45^{\circ}$ fiber orientation and a total displacement on the left boundary of 4.6 [mm]. Note 


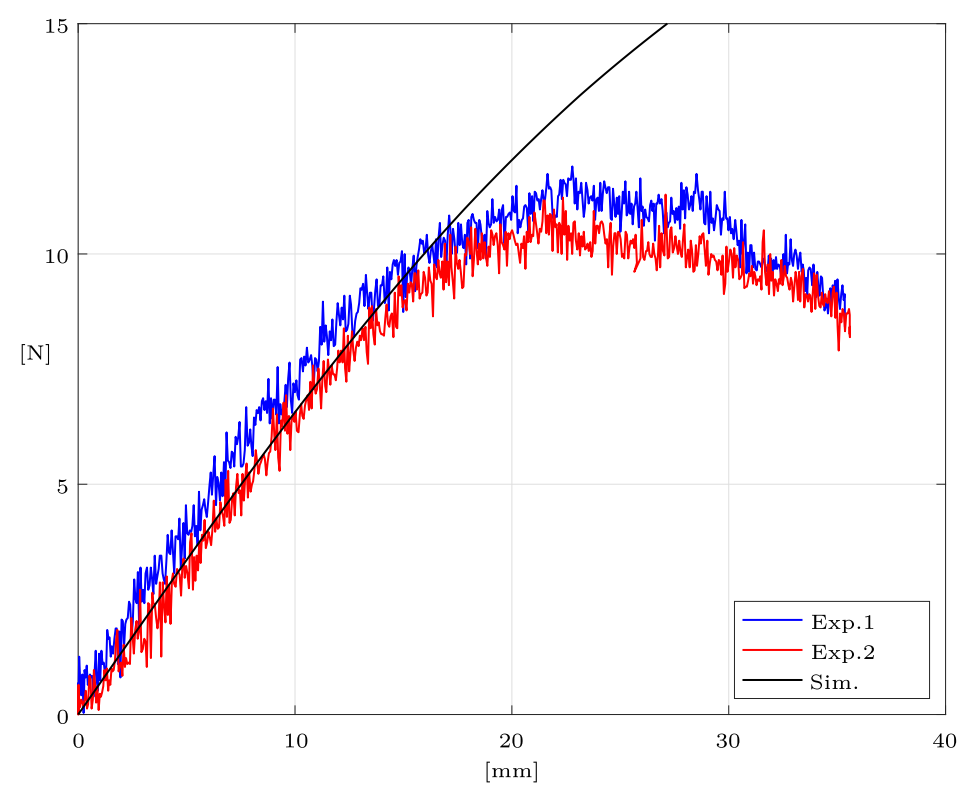

Fig. 22. Force-displacement curves of the out-of-plane bending test for a $25[\mathrm{~mm}]$ specimen with $0^{\circ}$ fiber orientation. Simulation results are compared with experimental investigations.

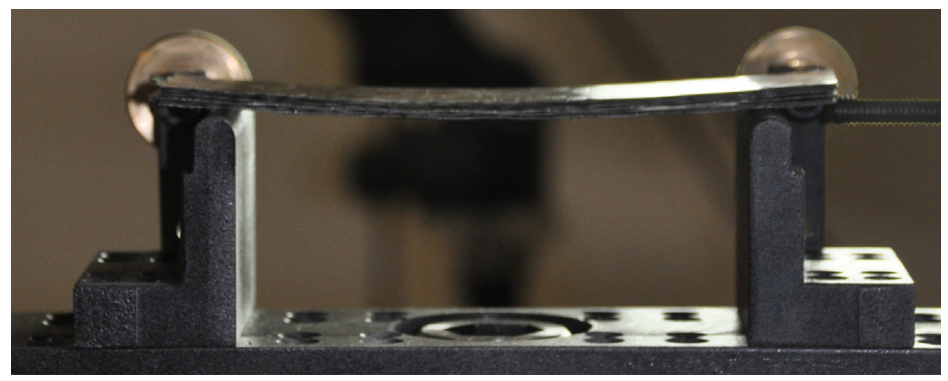

Fig. 23. 4-point bending test, permanent deformation of the sample after a full loading cycle.

that both sides are clamped in such a way that the displacement is prescribed and, additionally, the higher-order boundary conditions are also set, preventing in-plane twist and torsion of the fibers. A direct comparison with simulation results is not feasible, since the current model does not incorporate elastic softening or inelastic effects of the polymeric matrix material. However, we want to highlight the strain gradient effects in terms of the resulting deformation patterns. Therefore, the stiffness of the matrix material $E_{\text {iso }}$ and the bending stiffness $g_{1}$ and $g_{2}$ is reduced by a factor of 500 to fit the globally reduced stiffness of the inelastic deformed composite. ${ }^{3}$ In both plots in Fig. 24 the strain gradient effects result in the stiffness of the fibers against in-plane bending, which can be observed at the crosspoint of the fibers as highlighted by the red line.

Using the same material setting, we compare next results for a $36[\mathrm{~mm}]$ specimen in $30 / 60^{\circ}$ configuration, see Fig. 25. As can be seen, the deformation patterns fit in general, although the fibers at the vertices of the sample already start to fracture.

As final result of this section, we summarize the determined and validated material setting of our prototypical composite material Tepex ${ }^{\circledR}$ dynalite 102-RG600(1)/47 in Table 2.

\footnotetext{
3 The bending stiffness of the pure fiber is nearly zero, but contribute considerably to the global stiffness if the fibers are embedded within the matrix material. If the matrix is subject to inelastic effects, the bending stiffness is effected as well. Note that we have observed pronounced inelastic deformations by evaluating hysteresis curves. A detailed investigation of inelastic effects including plastification of the matrix material and fiber pullout is beyond scope of the current work, but will be part of a future contribution.
} 

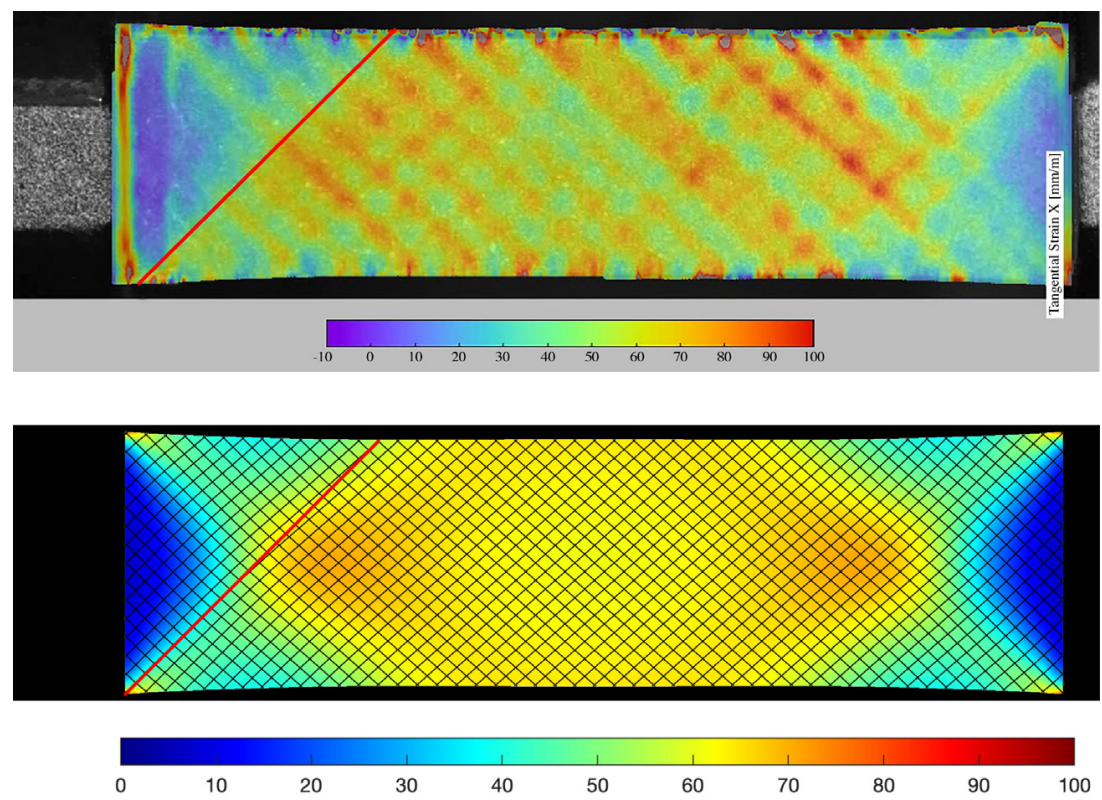

Fig. 24. DIC measurement of a $25[\mathrm{~mm}]$ specimen in $45^{\circ}$ configuration (top) and simulation results (bottom) at 4.6 [mm] total displacement, colors indicate local stretches in horizontal direction in $[\mathrm{mm} / \mathrm{m}]$. Black lines indicate the fiber direction in the current configuration. (For interpretation of the references to color in this figure legend, the reader is referred to the web version of this article.)
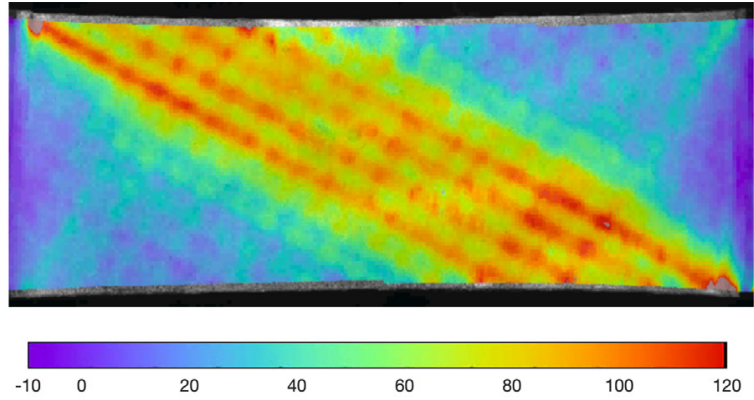
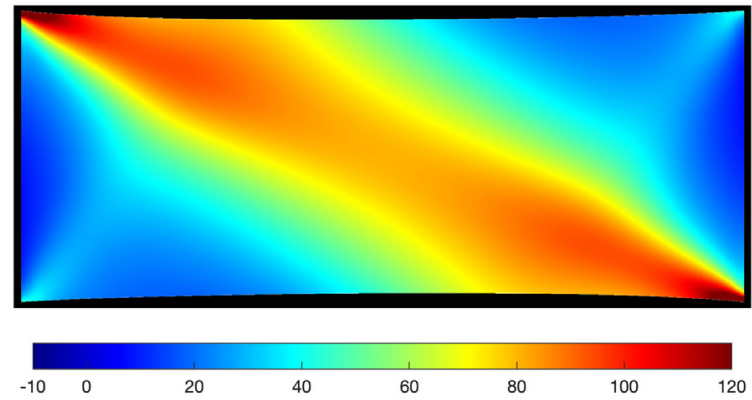

Fig. 25. DIC measurement of a 36 [mm] specimen in $60^{\circ}$ configuration (top) and simulation results (bottom) at 4.6 [mm] total displacement, colors indicate local stretches in horizontal direction in $[\mathrm{mm} / \mathrm{m}]$. (For interpretation of the references to color in this figure legend, the reader is referred to the web version of this article.)

Remark 4. Warp and weft direction are given in $0^{\circ}$ and $90^{\circ}$ direction, respectively. Both exhibit a slightly different material behavior and are not perfectly aligned due to the production process. This can be calibrated using different values for $a_{1 / 2}$ and $g_{1 / 2}$, which we omit here since the general behavior of this second gradient material is the key issue in this paper.

\subsection{Concluding example}

This last example demonstrates the applicability of the proposed model to complex geometries with non-planar reference configurations. Therefore we consider a shaft of length $50[\mathrm{~mm}]$ and radius $50 / \pi[\mathrm{mm}]$ as shown in Fig. 26, using the validated material setting given in Table 2. Additionally, we set $g_{3}=0$ and $k_{3}=0$. The discretization of the shaft consists of 200 cubic B-spline based elements. To obtain a closed cylinder, the extended Mortar method [40] is applied between the opposing edges in peripheral direction of the shell, maintaining $G^{1}$-continuity, see also Dittmann et al. [51,52] for more details. The displacement on the left in $\boldsymbol{e}_{2}$-direction of the cylinder is fixed in 
Table 2

Material setting of Tepex ${ }^{\circledR}$ dynalite 102-RG600(1)/47.

Compressible matrix material (PA 6)

Shear modulus $\mu$

$384.62\left[\mathrm{~N} / \mathrm{mm}^{2}\right]$

Bulk modulus $\kappa$

$833.33\left[\mathrm{~N} / \mathrm{mm}^{2}\right]$

Fiber material (roving glass)

Tensile stiffness $a_{1}, a_{2}$

Shear stiffness $a_{3}$

In-plane bending stiffness $g_{1}, g_{2}$

$8577.5[\mathrm{~N} / \mathrm{mm}]$

Out-of-plane bending stiffness $k_{1}, k_{2}$

$250[\mathrm{~N} / \mathrm{mm}]$

$893500[\mathrm{Nmm}]$

$78.949[\mathrm{Nmm}]$
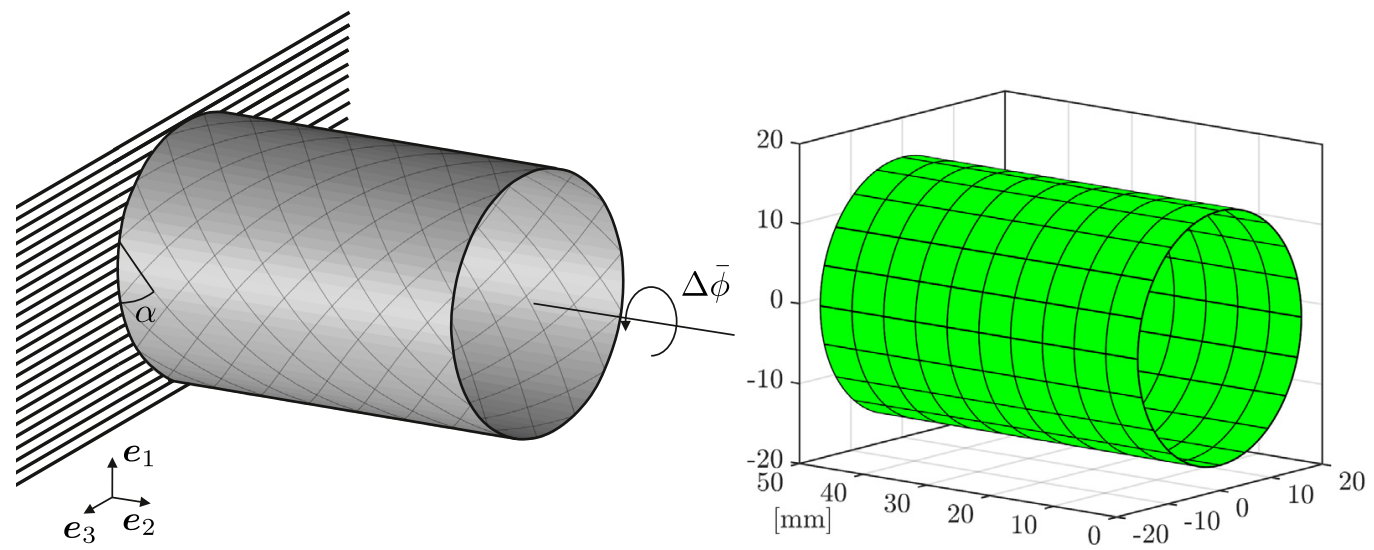

Fig. 26. Concluding Example. Problem setting and computational mesh.

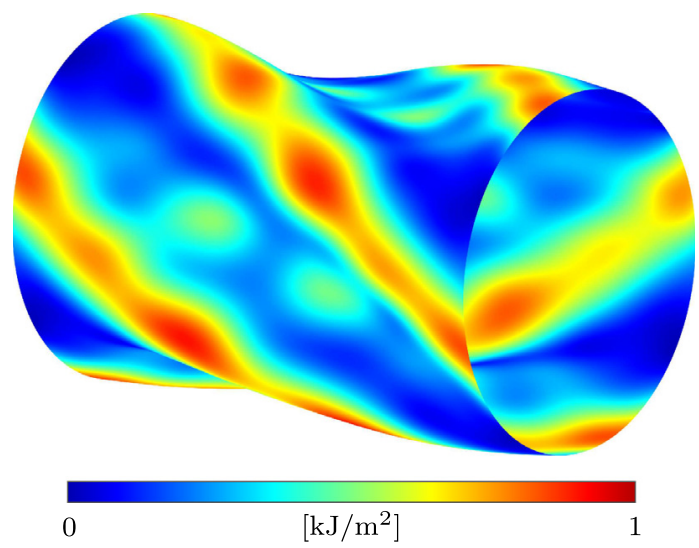

Fig. 27. Concluding Example. Strain energy density of the matrix material.

space, whereas the displacement on the right is fixed on the $\left(\boldsymbol{e}_{1}, \boldsymbol{e}_{3}\right)$-plane and rotated as illustrated in Fig. 26. Note that no higher order boundary conditions according to $(60)_{3}$ and (61) are applied.

First, we investigate a pure matrix material. After a prescribed angle of $\Delta \bar{\phi}=3.3^{\circ}$, the shell starts to buckle. The last state of equilibrium is obtained at an angle of $\Delta \bar{\phi}=12.6^{\circ}$, see Fig. 27. Note that e.g. an arc length method can be used to calculate further states in the geometrically unstable regime. In Fig. 28, the applied torque necessary to obtain the prescribed twist at the right hand side of the shell versus the angle of twist $\Delta \bar{\phi}$ is plotted for the pure matrix as well as for the composite material with different fiber orientations with and without the in-plane bending terms. 


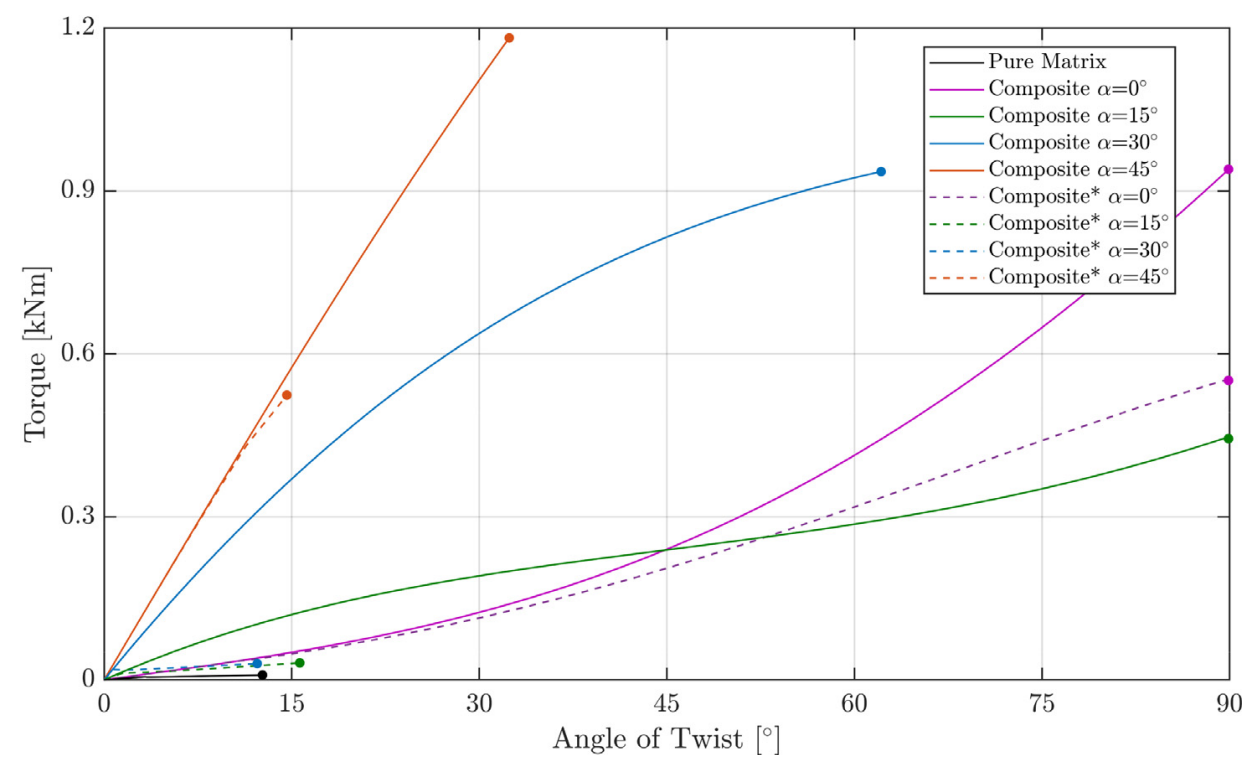

Fig. 28. Concluding Example. Torque versus angle of twist. Displayed are the results for the pure matrix material, the composite and the composite without the additional in-plane contributions (composite*).

Next, the composite material is investigated. In Fig. 29, upper left, the strain energy for a $0^{\circ}$ fiber configuration after a prescribed twist of $\Delta \bar{\phi}=90^{\circ}$ is displayed. Note that the fiber orientation and not the computational mesh is shown. The corresponding torque is given again in Fig. 28. Several issues can be highlighted here. First, the higher order boundary conditions as presented in $(60)_{3}$ are not set and thus, the fibers can twist in-plane at the boundary. This can be observed very well in Fig. 29, upper left. If bending would be prohibited at the boundary, the fibers have to remain orthogonal with regard to the boundary. Since these additional boundary conditions are unknown to a first gradient theory or even to the classical Kirchhoff-Love shell theory which only considers the out- and not the in-plane contributions, the proposed formulation is the only way to enforce this consistently within the mechanical formulation. Second, the in-plane terms stabilize the shell against buckling. Fig. 30, upper left, shows the results if the additional in-plane terms are not present, i.e. the material constants $g_{1}$ and $g_{2}$ are set to zero. Thus, we obtain the results for an anisotropic Kirchhoff-Love formulation, usual fitted via a first-gradient homogenization process, showing large buckling modes. To illustrate this further, the strain energy of the in-plane contributions for the deformed configuration (cf. Fig. 30, upper left) is plotted in Fig. 31. As can be observed, a dramatic increase of the in-plane strain energy occurs for this heavily deformed configuration, hence, in the mechanical equilibrium this buckling mode is not possible.

Additionally, results for $15^{\circ}, 30^{\circ}$ and $45^{\circ}$ fiber orientation are plotted (upper right, lower left and lower right picture in Figs. 29 and 30). The corresponding torque is plotted again in Fig. 28. Notably, a nearly constant strain energy distribution can be observed in Fig. 29, lower right and upper left.

\section{Conclusions}

In this paper, we formulate a Kirchhoff-Love shell theory to account for in-plane bending flexure resistance of embedded fibers. More specifically, the proposed formulation addresses in total additional twelve curvature components. Based on this set of deformation measures, we can now apply standard 3D constitutive laws to the shell continuum and combine them with the contribution of the woven fabric to describe composite materials in the hyperelastic regime. Moreover, the localization allows us to identify the corresponding boundary terms, which account for additional Dirichlet and Neumann contributions as required by fourth-order partial differential equation.

The verification and adjustment of the corresponding material parameters allows us to identify the physical effects of the higher-order terms in this prototypical second gradient material. Based on this verification step, we can now for the first time calibrate and validate a second gradient formulation using experimental results for fiber reinforced 

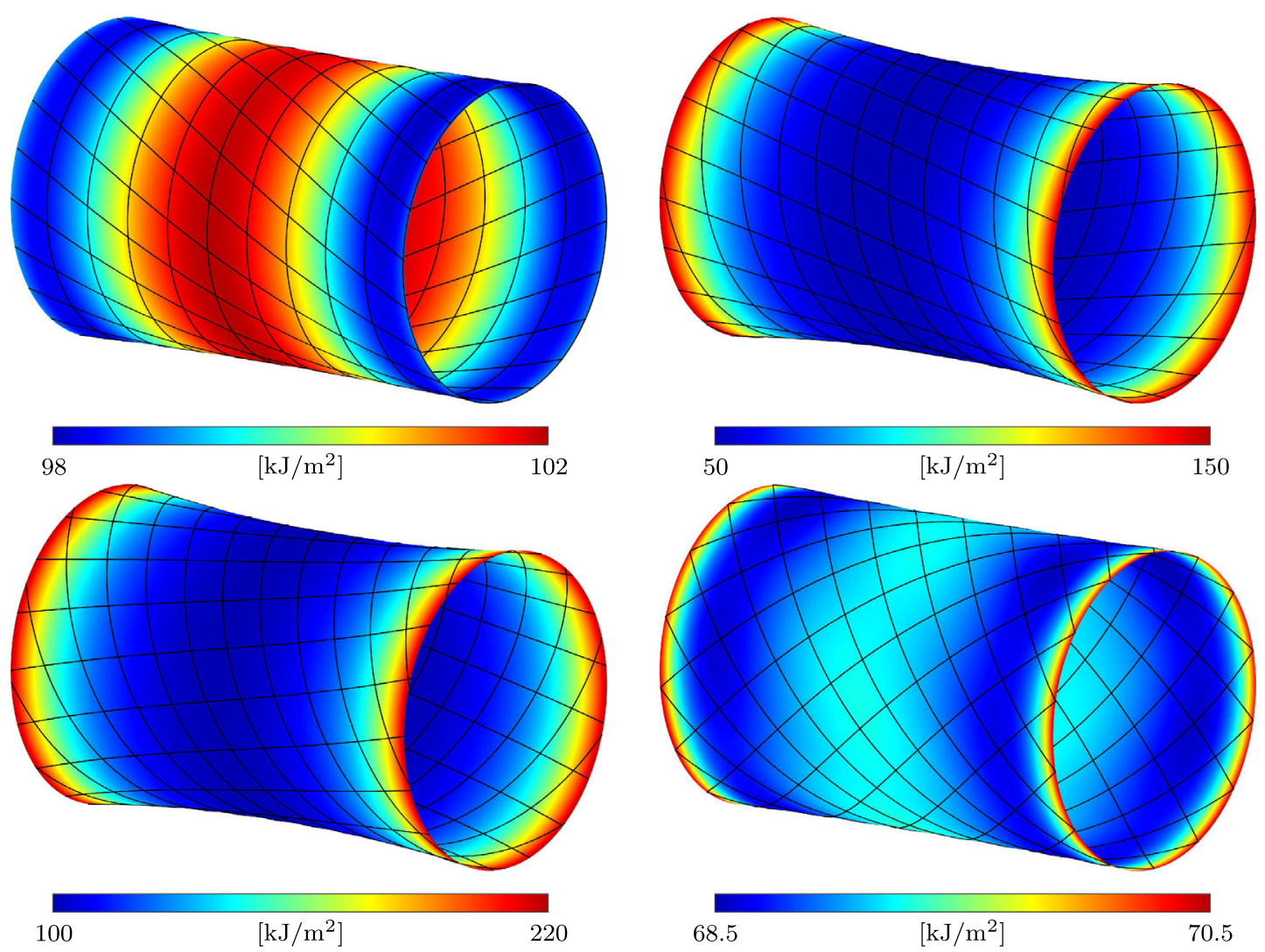

Fig. 29. Concluding Example. Strain energy density of the composite material with different fiber orientations $\alpha=\left[0^{\circ}, 15^{\circ}, 30^{\circ}, 45^{\circ}\right]($ from left to right and top to bottom) plotted at the final deformation state marked in Fig. 28.

material. This material is composed of a first gradient polymeric material and a second gradient woven fabric made of glass fiber.

Thus, we are now able to conduct simulations in the hyperelastic regime of this complex material behavior. Further developments will incorporate additional inelastic properties along with fracture mechanisms, as proposed e.g. in Dittmann et al. [53,54] and Aldakheel et al. [55].

\section{Acknowledgments}

Support for this research was provided by the Deutsche Forschungsgemeinschaft (DFG), Germany under grant HE5943/8-1. This support is gratefully acknowledged. The authors M. Dittmann and C. Hesch gratefully acknowledge support by the DFG, Germany under grant DI2306/1-1. The authors gratefully acknowledge the help of Heiko Bendler at the Institute of Mechanics (IFM) at the Karlsruhe Institute of Technology (KIT) to perform the experimental investigations on organic sheets. Finally, we thank an anonymous referee for detailed and helpful comments within the review process.

\section{Appendix A. Frame invariance}

To demonstrate the invariance of the introduced measures $\left\{a_{\alpha \beta}, \kappa_{\alpha, \beta}, S_{\alpha, \beta}^{\sigma}\right\}$ under superposed rigid body motions we define rigid motions of the form

$$
\boldsymbol{r}^{+}:=\boldsymbol{u}+\boldsymbol{Q r}
$$

where $\boldsymbol{u} \in \mathbb{E}^{3}$ and $\boldsymbol{Q} \in S O(3)$ is a rotation tensor with the property $\boldsymbol{Q}^{\mathrm{T}} \boldsymbol{Q}=\boldsymbol{I}$. Then, the first and second partial derivative read

$$
\boldsymbol{a}_{\alpha}^{+}=\boldsymbol{Q} \boldsymbol{r}_{, \alpha} \quad \text { and } \quad \boldsymbol{a}_{\alpha \beta}^{+}=\boldsymbol{Q} \boldsymbol{r}_{, \alpha \beta} .
$$



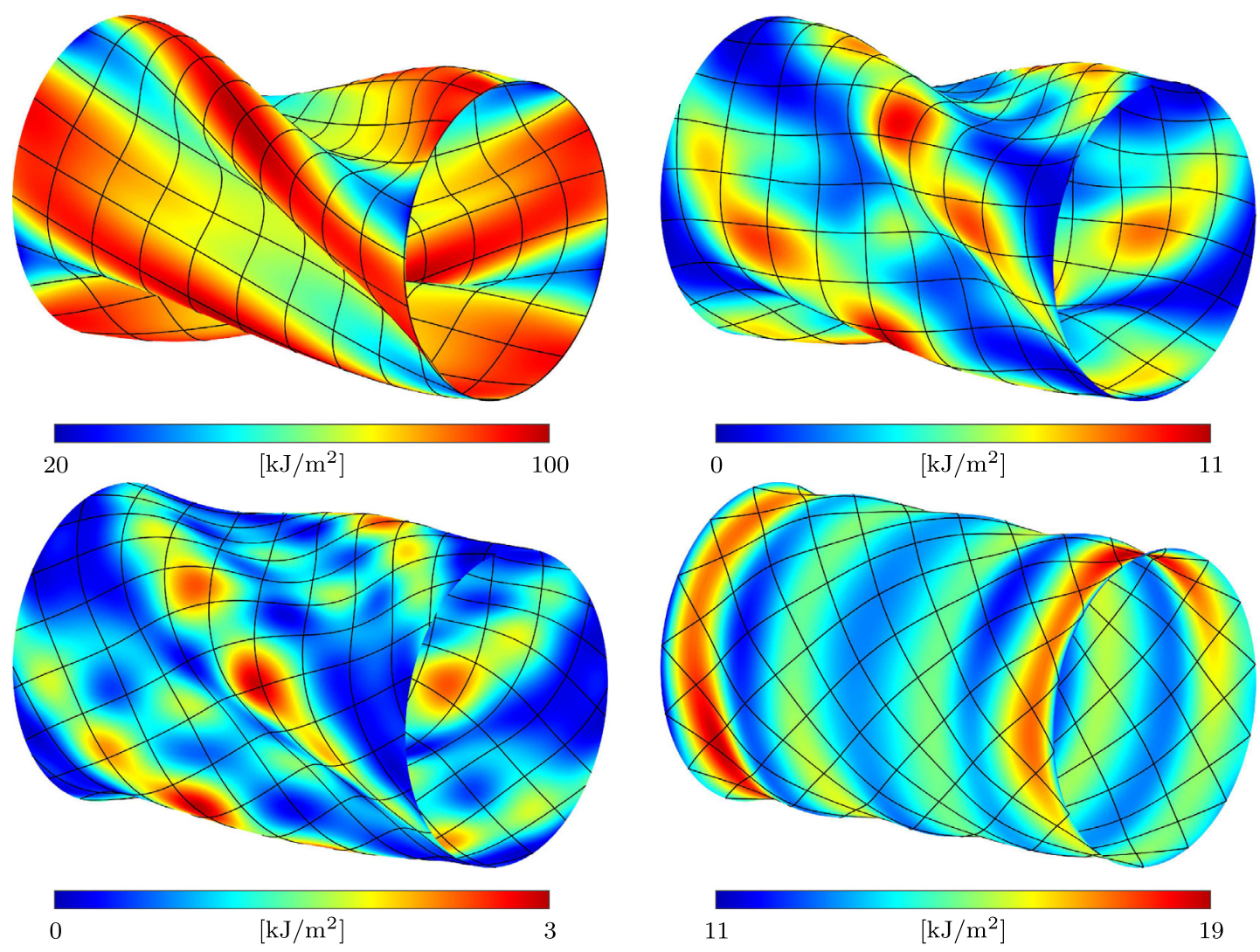

Fig. 30. Concluding Example. Strain energy density of the composite material with different fiber orientations $\alpha=\left[0^{\circ}, 15^{\circ}, 30^{\circ}, 45^{\circ}\right]($ from left to right and top to bottom) without in-plane contributions plotted at the final deformation state marked in Fig. 28.

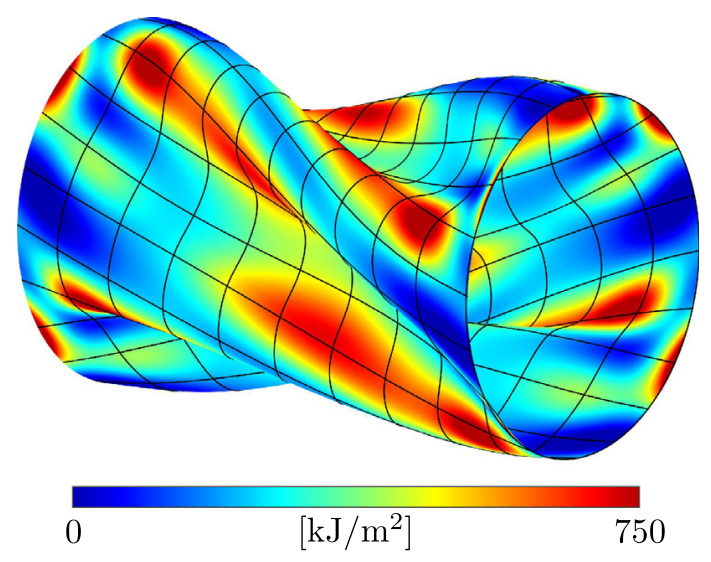

Fig. 31. Concluding Example. Estimated strain energy density of the in-plane contribution using the deformed configuration in 30 , upper left.

Starting with the covariant metric coefficients one gets

$$
a_{\alpha \beta}^{+}=\boldsymbol{a}_{\alpha}^{+} \cdot \boldsymbol{a}_{\beta}^{+}=\left(\boldsymbol{Q} \boldsymbol{r}_{, \alpha}\right) \cdot\left(\boldsymbol{Q} \boldsymbol{r}_{, \beta}\right)=\boldsymbol{r}_{, \alpha} \cdot \underbrace{\boldsymbol{Q}^{\mathrm{T}} \boldsymbol{Q}}_{=\boldsymbol{I}} \boldsymbol{r}_{, \beta}=a_{\alpha \beta}
$$


which clearly holds also for the contravariant metric coefficients, i.e. $\left(a^{\alpha \beta}\right)^{+}=a^{\alpha \beta}$. With respect to this result, we can show

$$
S_{\alpha \beta \lambda}^{+}=\Gamma_{\alpha \beta \lambda}^{+}-\bar{\Gamma}_{\alpha \beta}^{\lambda} a_{\alpha \lambda}^{+}=\left(\boldsymbol{Q} \boldsymbol{r}_{, \lambda}\right) \cdot\left(\boldsymbol{Q} \boldsymbol{r}_{, \alpha \beta}\right)-\bar{\Gamma}_{\alpha \beta}^{\lambda} a_{\alpha \lambda}=\boldsymbol{r}_{, \lambda} \cdot \underbrace{\boldsymbol{Q}^{\mathrm{T}} \boldsymbol{Q}}_{=\boldsymbol{I}} \boldsymbol{r}_{, \alpha \beta}-\bar{\Gamma}_{\alpha \beta}^{\lambda} a_{\alpha \lambda}=S_{\alpha \beta \lambda}
$$

as well as

$$
\left(S_{\alpha \beta}^{\sigma}\right)^{+}=S_{\alpha \beta \lambda}^{+}\left(a^{\sigma \lambda}\right)^{+}=S_{\alpha \beta \lambda} a^{\sigma \lambda}=S_{\alpha \beta}^{\sigma},
$$

i.e. the frame invariance of the co- and contravariant in-plane curvature tensor is confirmed. Eventually, we obtain for the out-of-plane curvature tensor

$$
\begin{aligned}
\kappa_{\alpha \beta}^{+} & =B_{\alpha \beta}-b_{\alpha \beta}^{+} \\
& =B_{\alpha \beta}-\boldsymbol{Q} \boldsymbol{r}_{, \alpha \beta} \cdot \frac{\boldsymbol{Q} \boldsymbol{a}_{1} \times \boldsymbol{Q} \boldsymbol{a}_{2}}{\| \boldsymbol{Q \boldsymbol { a } _ { 1 } \times \boldsymbol { Q } \boldsymbol { a } _ { 2 } \|}} \\
& =B_{\alpha \beta}-\frac{\operatorname{det}\left(\boldsymbol{Q} \boldsymbol{r}_{, \alpha \beta}, \boldsymbol{Q} \boldsymbol{a}_{1}, \boldsymbol{Q} \boldsymbol{a}_{2}\right)}{\sqrt{\operatorname{det}\left(a_{\alpha \beta}^{+}\right)}} \\
& =B_{\alpha \beta}-\underbrace{\operatorname{det}(\boldsymbol{Q})}_{=1} \frac{\operatorname{det}\left(\boldsymbol{r}_{, \alpha \beta}, \boldsymbol{a}_{1}, \boldsymbol{a}_{2}\right)}{\sqrt{\operatorname{det}\left(a_{\alpha \beta}\right)}} \\
& =\kappa_{\alpha \beta} .
\end{aligned}
$$

confirming again the frame invariance. Note that frame invariance of the deformation measures imply translational as well as rotational invariance of the strain energy which in turn implies satisfaction for conservation of linear and angular momentum, see Hesch \& Betsch [56] and Marsden \& Ratiu [57] for further details.

\section{Appendix B. Fiber deformation measures}

To obtain more insight into the fiber deformation measures (24)-(27), we derive them in the following from the conception that the fibers are modeled as mathematical curves with appropriate kinematical and constitutive structure to capture the mechanical properties of the reinforcement. Besides the applied formulation as functions of $a_{\alpha \beta}, S_{\alpha \beta}^{\sigma}, \kappa_{\alpha \beta}$ and $\theta^{\alpha}$, we show alternative representations in terms of stretch and curvature quantities given to mathematical curves.

The unit tangents to the fibers on $\omega$ are denoted by $\boldsymbol{l}$ and $\boldsymbol{m}$. Since these vectors are necessarily tangent to the mid-surface, they can be represented as

$$
\boldsymbol{l}=l^{\alpha} \boldsymbol{a}_{\alpha} \text { and } \boldsymbol{m}=m^{\alpha} \boldsymbol{a}_{\alpha} .
$$

For the description of the fibers in the reference mid-surface $\Omega$, we introduce the tangents $\boldsymbol{L}$ and $\boldsymbol{M}$, which do not necessarily have unit length but which are orthogonal to each other and can be written as

$$
\boldsymbol{L}=L^{\alpha} \boldsymbol{A}_{\alpha} \quad \text { and } \quad \boldsymbol{M}=M^{\alpha} \boldsymbol{A}_{\alpha} .
$$

Since the fibers are convected as material curves, the tangent vectors are related by

$$
\lambda_{1} \boldsymbol{l}=\boldsymbol{F} \frac{\boldsymbol{L}}{\|\boldsymbol{L}\|} \quad \text { and } \quad \lambda_{2} \boldsymbol{l}=\boldsymbol{F} \frac{\boldsymbol{M}}{\|\boldsymbol{M}\|}
$$

where we made use of the fiber stretches

$$
\lambda_{1}:=\left\|\frac{\boldsymbol{F} \boldsymbol{L}}{\|\boldsymbol{L}\|}\right\|=\frac{\sqrt{a_{\alpha \beta} L^{\alpha} L^{\beta}}}{\sqrt{A_{\mu \nu} L^{\mu} L^{\nu}}} \quad \text { and } \quad \lambda_{2}:=\left\|\frac{\boldsymbol{F} \boldsymbol{M}}{\|\boldsymbol{M}\|}\right\|=\frac{\sqrt{a_{\alpha \beta} M^{\alpha} M^{\beta}}}{\sqrt{A_{\mu \nu} M^{\mu} M^{\nu}}} .
$$

Therefore, the corresponding contravariant components are connected by

$$
L^{\alpha}=\lambda_{1}\|\boldsymbol{L}\| l^{\alpha}=\sqrt{a_{\mu \nu} L^{\mu} L^{\nu}} l^{\alpha} \quad \text { and } \quad M^{\alpha}=\lambda_{1}\|\boldsymbol{M}\| M^{\alpha}=\sqrt{a_{\mu \nu} M^{\mu} M^{\nu}} m^{\alpha} .
$$

The change of angle between the fibers can be easily obtained as

$$
\varphi:=\operatorname{acos}\left(\frac{A_{\alpha \beta} L^{\alpha} M^{\beta}}{\sqrt{A_{\mu \nu} L^{\mu} L^{\nu} A_{\sigma \rho} M^{\sigma} M^{\rho}}}\right)-\operatorname{acos}\left(\frac{a_{\alpha \beta} L^{\alpha} M^{\beta}}{\sqrt{a_{\mu \nu} L^{\mu} L^{\nu} a_{\sigma \rho} M^{\sigma} M^{\rho}}}\right) .
$$


For initially orthonormal fibers, the first term is certainly $\pi / 2$.

To study the change of the tangent vectors $\boldsymbol{l}$ and $\boldsymbol{m}$ within the deformed mid-surface, we introduce the auxiliary unit tangent vectors

$$
\boldsymbol{p}=\boldsymbol{n} \times \boldsymbol{l}, \quad \boldsymbol{q}=\boldsymbol{n} \times \boldsymbol{m} .
$$

Moreover, let $\tilde{\boldsymbol{l}}: \mathcal{B} \rightarrow \mathbb{E}^{3}$ and $\tilde{\boldsymbol{m}}: \mathcal{B} \rightarrow \mathbb{E}^{3}$ be such that

$$
\boldsymbol{l}\left(\theta^{\alpha}\right)=\tilde{\boldsymbol{l}}\left(\boldsymbol{r}\left(\boldsymbol{\theta}^{\alpha}\right)\right) \text { and } \boldsymbol{m}\left(\theta^{\alpha}\right)=\tilde{\boldsymbol{m}}\left(\boldsymbol{r}\left(\boldsymbol{\theta}^{\alpha}\right)\right) .
$$

Then the gradients

$$
\nabla \tilde{\boldsymbol{l}}(\boldsymbol{r})=\boldsymbol{l}_{, \alpha} \otimes \boldsymbol{a}^{\alpha} \quad \text { and } \quad \nabla \tilde{\boldsymbol{m}}(\boldsymbol{r})=\boldsymbol{m}_{, \alpha} \otimes \boldsymbol{a}^{\alpha}
$$

are easily obtained by inserting $\mathrm{d} \theta^{\alpha}=\mathrm{d} \boldsymbol{r} \cdot \boldsymbol{a}^{\alpha}$ into the expressions $\mathrm{d} \boldsymbol{l}=\boldsymbol{l}_{, \alpha} \mathrm{d} \theta^{\alpha}=\boldsymbol{l}_{, \alpha}\left(\mathrm{d} \boldsymbol{r} \cdot \boldsymbol{a}^{\alpha}\right)=\left(\boldsymbol{l}_{, \alpha} \otimes \boldsymbol{a}^{\alpha}\right) \mathrm{d} \boldsymbol{r}$ and $\mathrm{d} \boldsymbol{m}=\boldsymbol{m}_{, \alpha} \mathrm{d} \theta^{\alpha}=\left(\boldsymbol{m}_{, \alpha} \otimes \boldsymbol{a}^{\alpha}\right) \mathrm{d} \boldsymbol{r}$, respectively. Since $\|\boldsymbol{l}\|=\|\boldsymbol{m}\|=1$, we have $\boldsymbol{l} \cdot \boldsymbol{l}_{, \alpha}=\boldsymbol{m} \cdot \boldsymbol{m}_{, \alpha}=0$ and the partial derivatives of the unit vectors $\boldsymbol{l}$ and $\boldsymbol{m}$ can be expressed in the right-handed orthonormal bases $\{\boldsymbol{l}, \boldsymbol{p}, \boldsymbol{n}\}$ and $\{\boldsymbol{m}, \boldsymbol{q}, \boldsymbol{n}\}$ as

$$
\boldsymbol{l}_{, \alpha}=\left(\boldsymbol{l}_{, \alpha} \cdot \boldsymbol{p}\right) \boldsymbol{p}+\left(\boldsymbol{l}_{, \alpha} \cdot \boldsymbol{n}\right) \boldsymbol{n} \quad \text { and } \quad \boldsymbol{m}_{, \alpha}=\left(\boldsymbol{m}_{, \alpha} \cdot \boldsymbol{q}\right) \boldsymbol{q}+\left(\boldsymbol{m}_{, \alpha} \cdot \boldsymbol{n}\right) \boldsymbol{n} .
$$

Using (87) together with the Gauss and Weingarten equation (6), the normal components in (96) can be expressed using the covariant components of the mid-surface curvature $b_{\alpha \beta}$ as

$$
\boldsymbol{l}_{, \alpha} \cdot \boldsymbol{n}=\left(l^{\beta} \boldsymbol{a}_{\beta}\right)_{, \alpha} \cdot \boldsymbol{n}=l^{\beta} b_{\beta \alpha} \quad \text { and } \quad \boldsymbol{m}_{, \alpha} \cdot \boldsymbol{n}=\left(m^{\beta} \boldsymbol{a}_{\beta}\right)_{, \alpha} \cdot \boldsymbol{n}=m^{\beta} b_{\beta \alpha} .
$$

The change of the unit tangent vectors in tangential direction can therefore be represented by

$$
\nabla \tilde{\boldsymbol{l}}(\boldsymbol{r}) \boldsymbol{l}=l^{\alpha} \boldsymbol{l}_{, \alpha}=\eta_{l} \boldsymbol{p}+\kappa_{l} \boldsymbol{n} \quad \text { and } \quad \nabla \tilde{\boldsymbol{m}}(\boldsymbol{r}) \boldsymbol{m}=m^{\alpha} \boldsymbol{m}_{, \alpha}=\eta_{m} \boldsymbol{q}+\kappa_{m} \boldsymbol{n}
$$

with the normal fiber curvatures

$$
\kappa_{l}:=b_{\alpha \beta} l^{\alpha} l^{\beta}=\frac{b_{\alpha \beta} L^{\alpha} L^{\beta}}{a_{\mu \nu} L^{\mu} L^{\nu}} \quad \text { and } \quad \kappa_{m}:=b_{\alpha \beta} m^{\alpha} m^{\beta}=\frac{b_{\alpha \beta} M^{\alpha} M^{\beta}}{a_{\mu \nu} M^{\mu} M^{\nu}}
$$

and the geodesic curvatures

$$
\eta_{l}:=l^{\alpha} \boldsymbol{l}_{, \alpha} \cdot \boldsymbol{p} \quad \text { and } \quad \eta_{m}:=m^{\alpha} \boldsymbol{m}_{, \alpha} \cdot \boldsymbol{q} .
$$

The change of the unit tangent vectors of one fiber family in direction of the other fiber family leads to

$$
\nabla \tilde{\boldsymbol{l}}(\boldsymbol{r}) \boldsymbol{m}=m^{\alpha} \boldsymbol{l}_{, \alpha}=\phi_{l} \boldsymbol{p}+\tau \boldsymbol{n} \quad \text { and } \quad \nabla \tilde{\boldsymbol{m}}(\boldsymbol{r}) \boldsymbol{m}=m^{\alpha} \boldsymbol{m}_{, \alpha}=\phi_{m} \boldsymbol{q}+\tau \boldsymbol{n},
$$

where we recognize the torsion and the respective Tchebychev curvatures, [18],

$$
\tau:=b_{\alpha \beta} l^{\alpha} m^{\beta}=\frac{b_{\alpha \beta} L^{\alpha} M^{\beta}}{\sqrt{a_{\mu \nu} L^{\mu} L^{\nu} a_{\sigma \rho} M^{\sigma} M^{\rho}}}, \quad \phi_{l}:=m^{\alpha}\left(\boldsymbol{l}_{, \alpha} \cdot \boldsymbol{p}\right), \quad \phi_{m}:=l^{\alpha}\left(\boldsymbol{m}_{, \alpha} \cdot \boldsymbol{q}\right) .
$$

Similarly to (99) and (102), we can introduce the normal fiber curvatures $\kappa_{L}, \kappa_{M}$ and the torsion $\bar{\tau}$ on the reference mid-surface as

$$
\kappa_{L}:=\frac{B_{\alpha \beta} L^{\alpha} L^{\beta}}{A_{\mu \nu} L^{\mu} L^{\nu}}, \quad \kappa_{M}:=\frac{B_{\alpha \beta} M^{\alpha} M^{\beta}}{A_{\mu \nu} M^{\mu} M^{\nu}} \quad \text { and } \quad \bar{\tau}:=\frac{B_{\alpha \beta} L^{\alpha} M^{\beta}}{\sqrt{A_{\mu \nu} L^{\mu} L^{\nu} A_{\sigma \rho} M^{\sigma} M^{\rho}}} .
$$

Because these definitions rely on unit tangent vectors, the normalization by $\|\boldsymbol{L}\|$ and $\|\boldsymbol{M}\|$ are involved in here.

As deformation measures that captures the change in the normal curvature of the fibers, i.e. by out-of-plane bending, we choose

$$
K_{1}:=-\frac{\kappa_{\alpha \beta} L^{\alpha} L^{\beta}}{A_{\mu \nu} L^{\mu} L^{\nu}}=\lambda_{1}^{2} \kappa_{l}-\kappa_{L} \quad \text { and } \quad K_{2}:=-\frac{\kappa_{\alpha \beta} M^{\alpha} M^{\beta}}{A_{\mu \nu} M^{\mu} M^{\nu}}=\lambda_{1}^{2} \kappa_{m}-\kappa_{M}
$$

The change in torsion by twisting the fibers is given by

$$
K_{3}:=-\frac{\kappa_{\alpha \beta} L^{\alpha} M^{\beta}}{\sqrt{A_{\mu \nu} L^{\mu} L^{\nu} A_{\sigma \rho} M^{\sigma} M^{\rho}}}=\lambda_{1} \lambda_{2} \tau-\bar{\tau} .
$$


Finally, to describe the deformation of the fibers within the tangent planes of the mid-surfaces, like in-plane-bending, we introduce as strain measures

$$
\boldsymbol{g}_{L}:=\frac{L^{\alpha} L^{\beta} S_{\alpha \beta \sigma}}{A_{\mu \nu} L^{\mu} L^{\nu}} \boldsymbol{a}^{\sigma}, \quad \boldsymbol{g}_{M}:=\frac{M^{\alpha} M^{\beta} S_{\alpha \beta \sigma}}{A_{\mu \nu} M^{\mu} M^{\nu}} \boldsymbol{a}^{\sigma} \quad \text { and } \quad \boldsymbol{\Gamma}:=\frac{L^{\alpha} M^{\beta} S_{\alpha \beta \sigma}}{\sqrt{A_{\mu \nu} L^{\mu} L^{\nu} A_{\sigma \rho} M^{\sigma} M^{\rho}}} \boldsymbol{a}^{\sigma} .
$$

For initially straight fibers, the deformation measures (106) can be represented as

$$
\begin{aligned}
\boldsymbol{g}_{L} & =\lambda_{1} \eta_{l} \boldsymbol{p}+\left(\boldsymbol{L} \cdot \nabla \lambda_{1}\right) \boldsymbol{l}, \quad \boldsymbol{g}_{M}=\lambda_{2} \eta_{m} \boldsymbol{q}+\left(\boldsymbol{M} \cdot \nabla \lambda_{2}\right) \boldsymbol{m} \\
\boldsymbol{\Gamma} & =\left(\boldsymbol{L} \cdot \nabla \lambda_{2}\right) \boldsymbol{m}+\lambda_{1} \lambda_{2} \phi_{m} \boldsymbol{q}=\left(\boldsymbol{M} \cdot \nabla \lambda_{1}\right) \boldsymbol{l}+\lambda_{1} \lambda_{2} \phi_{l} \boldsymbol{p},
\end{aligned}
$$

with the stretch gradients as $\nabla \lambda_{1}=\lambda_{1, \alpha} A^{\alpha}$ and $\nabla \lambda_{2}=\lambda_{2, \alpha} A^{\alpha}$ (see Equation (58) and (59) in [20]). We refer to Section 2.4 of [20], where also the general precurved situation is analyzed. However, the restriction to straight fibers lies only in the representation (107), which should help to get more insight into strain measures (106). In fact, these strain measures obtained by the contraction of the fiber tangents of the reference mid-surface with the third order tensor $\boldsymbol{a}_{\sigma} \otimes \boldsymbol{S}^{\sigma}$ represent a combination of the gradients of the fiber stretch and in-plane-deformation of the fibers described by geodesic and Tchebychev curvatures.

\section{Appendix C. Stress resultants}

Applying the strain energy function of the matrix material given in (41) and using $\left[\hat{C}^{\alpha \beta}\right]=\left[\hat{C}_{\alpha \beta}\right]^{-1}$ together with

$$
\frac{\partial}{\partial \hat{C}_{\mu \nu}}\left(\frac{1}{\operatorname{det}\left(\hat{C}_{\alpha \beta}\right)}\right)=-\frac{1}{\operatorname{det}\left(\hat{C}_{\alpha \beta}\right)^{2}} \operatorname{det}\left(\hat{C}_{\lambda \sigma}\right) \hat{C}^{\mu \nu}=-\frac{\hat{C}^{\mu \nu}}{\operatorname{det}\left(\hat{C}_{\alpha \beta}\right)}
$$

we get

$$
\frac{\partial \Psi^{\text {iso }}}{\partial \hat{C}_{\mu \nu}}=\frac{1}{2} \mu\left(G^{\mu \nu}-\frac{\operatorname{det}\left(G_{\mu \nu}\right)}{\operatorname{det}\left(\hat{C}_{\alpha \beta}\right)} \hat{C}^{\mu \nu}\right)
$$

that has to be integrated in thickness direction to obtain the stress resultants.

The corresponding stress resultants of the first term $(36)_{1}$ reads

$$
\begin{aligned}
& n_{\mathrm{fib}}^{\alpha \beta}=\frac{\partial \Psi^{\mathrm{fib}}}{\partial \lambda_{1}} \frac{\partial \lambda_{1}}{\partial a_{\alpha \beta}}+\frac{\partial \Psi^{\mathrm{fib}}}{\partial \lambda_{2}} \frac{\partial \lambda_{2}}{\partial a_{\alpha \beta}}+\frac{\partial \Psi^{\mathrm{fib}}}{\partial \varphi} \frac{\partial \varphi}{\partial a_{\alpha \beta}}+\frac{\partial \Psi^{\mathrm{fib}}}{\partial C^{\sigma \mu}} \frac{\partial C^{\sigma \mu}}{\partial a_{\alpha \beta}} \\
&=a_{1}\left(\lambda_{1}-1\right) \frac{\partial \lambda_{1}}{\partial a_{\alpha \beta}}+a_{2}\left(\lambda_{2}-1\right) \frac{\partial \lambda_{2}}{\partial a_{\alpha \beta}}+2 a_{3} \frac{\tan (\varphi)}{\cos ^{2}(\varphi)} \frac{\partial \varphi}{\partial a_{\alpha \beta}} \\
&+\left(g_{1} \frac{\left(L^{\gamma} L^{\lambda} S_{\gamma \lambda \sigma}\right)\left(L^{\zeta} L^{\iota} S_{\zeta \iota \mu}\right)}{\left(A_{\mu \nu} L^{\mu} L^{\nu}\right)^{2}}+g_{2} \frac{\left(M^{\gamma} M^{\lambda} S_{\gamma \lambda \sigma}\right)\left(M^{\zeta} M^{\iota} S_{\zeta \iota \mu}\right)}{\left(A_{\mu \nu} M^{\mu} M^{\nu}\right)^{2}}\right. \\
&\left.+g_{3} \frac{\left(L^{\gamma} M^{\lambda} S_{\gamma \lambda \sigma}\right)\left(L^{\zeta} M^{\iota} S_{\zeta \iota \mu}\right)}{A_{\mu \nu} L^{\mu} L^{\nu} A_{\sigma \rho} M^{\sigma} M^{\rho}}\right) \frac{\partial C^{\sigma \mu}}{\partial a_{\alpha \beta}}
\end{aligned}
$$

where we have made use of (42) as strain energy function of the fiber material and

$$
\begin{gathered}
\boldsymbol{g}_{L} \cdot \boldsymbol{g}_{L}=\frac{\left(L^{\alpha} L^{\beta} S_{\alpha \beta \sigma}\right)\left(L^{\gamma} L^{\lambda} S_{\gamma \lambda \mu}\right) C^{\sigma \mu}}{\left(A_{\mu \nu} L^{\mu} L^{v}\right)^{2}}, \\
\boldsymbol{g}_{M} \cdot \boldsymbol{g}_{M}=\frac{\left(M^{\alpha} M^{\beta} S_{\alpha \beta \sigma}\right)\left(M^{\gamma} M^{\lambda} S_{\gamma \lambda \mu}\right) C^{\sigma \mu}}{\left(A_{\mu \nu} M^{\mu} M^{\nu}\right)^{2}}, \\
\boldsymbol{\Gamma} \cdot \boldsymbol{\Gamma}=\frac{\left(L^{\alpha} M^{\beta} S_{\alpha \beta \sigma}\right)\left(L^{\gamma} M^{\lambda} S_{\gamma \lambda \mu}\right) C^{\sigma \mu}}{A_{\mu \nu} L^{\mu} L^{\nu} A_{\sigma \rho} M^{\sigma} M^{\rho}}
\end{gathered}
$$

Moreover, the relations

$$
\frac{\partial \lambda_{1}}{\partial a_{\alpha \beta}}=\frac{1}{2} \frac{L^{\alpha} L^{\beta}}{\sqrt{a_{\sigma \lambda} L^{\sigma} L^{\lambda} A_{\mu \nu} L^{\mu} L^{\nu}}}, \quad \frac{\partial \lambda_{2}}{\partial a_{\alpha \beta}}=\frac{1}{2} \frac{M^{\alpha} M^{\beta}}{\sqrt{a_{\sigma \lambda} M^{\sigma} M^{\lambda} A_{\mu \nu} M^{\mu} M^{\nu}}}
$$


and

$$
\begin{aligned}
\frac{\partial \varphi}{a_{\alpha \beta}}= & \frac{1}{\sqrt{1-\frac{\left(a_{\alpha \beta} L^{\alpha} M^{\beta}\right)^{2}}{a_{\mu \nu} L^{\mu} L^{v} a_{\sigma \rho} M^{\sigma} M^{\rho}}}}\left(\frac{L^{\alpha} M^{\beta}}{\sqrt{a_{\mu \nu} L^{\mu} L^{\nu} a_{\sigma \rho} M^{\sigma} M^{\rho}}}\right. \\
& \left.-\frac{a_{\alpha \beta} L^{\alpha} M^{\beta}}{2\left(a_{\mu \nu} L^{\mu} L^{v} a_{\sigma \rho} M^{\sigma} M^{\rho}\right)^{3 / 2}}\left(a_{\mu \nu} L^{\mu} L^{\nu} M^{\sigma} M^{\rho}+L^{\mu} L^{\nu} a_{\sigma \rho} M^{\sigma} M^{\rho}\right)\right)
\end{aligned}
$$

as well as the partial derivative of the contravariant metric

$$
\frac{\partial C^{\sigma \mu}}{\partial a_{\alpha \beta}}=\frac{\partial C^{\sigma \mu}}{\partial C_{\alpha \beta}}=-C^{\sigma \alpha} C^{\beta \mu}
$$

have been used. For the second term $(36)_{2}$, the stress resultants of the out-of-plane bending moments reads

$$
m_{\mathrm{fib}}^{\alpha \beta}=-k_{1} K_{1} \frac{L^{\alpha} L^{\beta}}{A_{\mu \nu} L^{\mu} L^{\nu}}-k_{2} K_{2} \frac{M^{\alpha} M^{\beta}}{A_{\mu \nu} M^{\mu} M^{\nu}}-k_{3} K_{3} \frac{L^{\alpha} M^{\beta}}{\sqrt{A_{\mu \nu} L^{\mu} L^{\nu} A_{\sigma \rho} M^{\sigma} M^{\rho}}},
$$

whereas the third term $(36)_{3}$ yields

$$
\begin{aligned}
m_{\mathrm{fib}}^{\alpha \beta \sigma}= & g_{1} \frac{L^{\alpha} L^{\beta}\left(L^{\gamma} L^{\lambda} S_{\gamma \lambda \mu}\right)}{\left(A_{\mu \nu} L^{\mu} L^{\nu}\right)^{2}} C^{\sigma \mu}+g_{2} \frac{M^{\alpha} M^{\beta}\left(M^{\gamma} M^{\lambda} S_{\gamma \lambda \mu}\right)}{\left(A_{\mu \nu} M^{\mu} M^{\nu}\right)^{2}} C^{\sigma \mu}+ \\
& g_{3} \frac{L^{\alpha} M^{\beta}\left(L^{\gamma} M^{\lambda} S_{\gamma \lambda \mu}\right)}{A_{\mu \nu} L^{\mu} L^{\nu} A_{\sigma \rho} M^{\sigma} M^{\rho}} C^{\sigma \mu} .
\end{aligned}
$$

\section{Appendix D. Analytics - shear test}

Assuming a linear parametrization of the reference mid-surface

$$
\overline{\boldsymbol{r}}=\theta^{1} \boldsymbol{e}_{1}+\theta^{2} \boldsymbol{e}_{2} \quad \text { with } \theta^{1} \in[0, l] \text { and } \theta^{2} \in[0, b],
$$

the analytical solution for pure fiber material with a fiber configuration of $\alpha=0^{\circ}$ can be derived as follows. Note that we obtain $\boldsymbol{A}_{1}=\boldsymbol{e}_{1}$ and $\boldsymbol{A}_{2}=\boldsymbol{e}_{2}$ due to (117). The isochoric deformed configuration of the mid-surface shown in Fig. 8 is described by

$$
\boldsymbol{r}\left(\theta^{1}, \theta^{2}\right)=\left(\theta^{1}+\frac{\theta^{2} u}{b}\right) \boldsymbol{e}_{1}+\theta^{2} \boldsymbol{e}_{2},
$$

where $u$ is the predefined displacement of the upper boundary, see Fig. 7. Now, applying (4), (5), (24) and (25) we obtain

$$
\lambda_{1}=1, \quad \lambda_{2}=\sqrt{\left(\frac{u}{b}\right)^{2}+1} \quad \text { and } \varphi=\frac{\pi}{2}-\operatorname{acos}\left(\frac{\frac{u}{b}}{\sqrt{\left(\frac{u}{b}\right)^{2}+1}}\right)
$$

as fiber stretches and the change of angle between the fibers, respectively. Insertion of the strain measures into (42) and (60) yields

$$
\Psi^{\mathrm{fib}}=\frac{a_{2}}{2}\left(\sqrt{\left(\frac{u}{b}\right)^{2}+1}-1\right)^{2}+\frac{a_{3}}{2} \tan \left(\frac{\pi}{2}-\operatorname{acos}\left(\frac{\frac{u}{b}}{\sqrt{\left(\frac{u}{b}\right)^{2}+1}}\right)\right)^{2}
$$

and after some straightforward calculations

$$
N^{1}=a_{3} \Pi\left[\begin{array}{l}
0 \\
1 \\
0
\end{array}\right] \text { and } \quad \boldsymbol{N}^{2}=a_{2}\left(1-\frac{1}{\sqrt{\left(\frac{u}{b}\right)^{2}+1}}\right)\left[\begin{array}{l}
\frac{u}{b} \\
1 \\
0
\end{array}\right]+a_{3} \frac{\Pi}{\left(\frac{u}{b}\right)^{2}+1}\left[\begin{array}{c}
1 \\
-\frac{u}{b} \\
0
\end{array}\right],
$$


where the abbreviation

$$
\Pi=\frac{\tan \left(\frac{\pi}{2}-\operatorname{acos}\left(\frac{\frac{u}{b}}{\sqrt{\left(\frac{u}{b}\right)^{2}+1}}\right)\right)}{\cos \left(\frac{\pi}{2}-\operatorname{acos}\left(\frac{\frac{u}{b}}{\sqrt{\left(\frac{u}{b}\right)^{2}+1}}\right)\right)^{2}} .
$$

is applied. Eventually, using (60) we can evaluate the resultant forces at the right boundary $\Upsilon_{1}$ and upper boundary $\Upsilon_{2}$ as

$$
\boldsymbol{F}_{\Upsilon_{1}}=\int_{\Upsilon_{1}}\left[\begin{array}{ll}
N^{1} & N^{2}
\end{array}\right] \boldsymbol{v}_{\Upsilon_{1}} \mathrm{~d} S=N^{1} b \quad \text { and } \quad \boldsymbol{F}_{\Upsilon_{2}}=\int_{\Upsilon_{2}}\left[\begin{array}{ll}
\boldsymbol{N}^{1} & \boldsymbol{N}^{2}
\end{array}\right] \boldsymbol{v} \Upsilon_{2} \mathrm{~d} S=\boldsymbol{N}^{2} l,
$$

respectively, where $\boldsymbol{v}_{\Upsilon_{1}}=[1,0]^{\mathrm{T}}$ and $\boldsymbol{v}_{\Upsilon_{2}}=[0,1]^{\mathrm{T}}$ are corresponding unit normal vectors. Thus, for the final deformation state, i.e. $u / b=1$, we obtain

$$
\Psi^{\mathrm{fib}}(u=0.1[\mathrm{~m}]) \approx 3029.3 \frac{\mathrm{J}}{\mathrm{m}^{2}}
$$

as homogeneous strain energy distribution and the forces at the boundaries are

$$
\boldsymbol{F}_{\Upsilon_{1}}(u=0.1[\mathrm{~m}])=\left[\begin{array}{c}
0 \\
800 \\
0
\end{array}\right][\mathrm{N}] \quad \text { and } \quad \boldsymbol{F}_{\Upsilon_{2}}(u=0.1[\mathrm{~m}]) \approx\left[\begin{array}{c}
-72.8 \\
1127.2 \\
0
\end{array}\right][\mathrm{N}]
$$

\section{Appendix E. Analytics - torsion test}

Assuming a linear parametrization of the reference mid-surface, cf. (117), with $\theta^{1} \in[0, l]$ and $\theta^{2} \in[-b / 2, b / 2]$ such that $\boldsymbol{A}_{1}=\boldsymbol{e}_{1}$ and $\boldsymbol{A}_{2}=\boldsymbol{e}_{2}$, strain energy distributions for pure fiber material with different fiber configurations can be evaluated as follows. The predefined deformation of the mid-surface shown in Figs. 16 and 17, respectively, is described by

$$
\boldsymbol{r}\left(\theta^{1}, \theta^{2}\right)=\theta^{1} \boldsymbol{e}_{1}+\theta^{2} \cos \left(\frac{\pi \theta^{1}}{l}\right) \boldsymbol{e}_{2}+\theta^{2} \sin \left(\frac{\pi \theta^{1}}{l}\right) \boldsymbol{e}_{3} .
$$

Applying (4), (7) 2 and (23) 2 we obtain

$$
\kappa_{11}=\kappa_{22}=0 \quad \text { and } \quad \kappa_{12}=\kappa_{21}=-\frac{\pi}{\sqrt{\left(\theta^{2} \pi\right)^{2}+l^{2}}} .
$$

Concerning different fiber configurations defined by $\alpha$, the vector fields $\boldsymbol{L}$ and $\boldsymbol{M}$ in the reference configuration are given by

$$
L^{1}=\cos (\alpha), \quad L^{2}=\sin (\alpha), \quad M^{1}=-\sin (\alpha), \quad M^{2}=\cos (\alpha) .
$$

Eventually, insertion of these derivations into (26) 3 and (42) yields

$$
\Psi^{\mathrm{fib}}=\frac{k_{3}}{2}\left(K_{3}\right)^{2}=\frac{k_{3}}{2} \frac{\pi^{2}}{\left(\theta^{2} \pi\right)^{2}+l^{2}}\left(\cos (\alpha)^{2}-\sin (\alpha)^{2}\right)^{2} .
$$

Note that for the evaluation all material parameters are set equal to zero excepted $k_{3}$. Obviously, for the $\alpha=45^{\circ}$ fiber configuration, the strain energy distribution related to a torsional deformation of the shell is identical to zero, whereas for each other configuration, the highest value is obtained at the central axis of the shell, i.e. at $\theta^{2}=0$. We obtain for the $\alpha=0^{\circ}$ fiber configuration

$$
\Psi^{\mathrm{fib}}\left(\theta^{2}=0\right) \approx 1097.06 \frac{\mathrm{J}}{\mathrm{m}^{2}} \quad \text { and } \quad \Psi^{\mathrm{fib}}\left(\theta^{2}=b / 2\right)=\Psi^{\mathrm{fib}}\left(\theta^{2}=-b / 2\right) \approx 523.25 \frac{\mathrm{J}}{\mathrm{m}^{2}},
$$

for the $\alpha=15^{\circ}$ fiber configuration

$$
\Psi^{\mathrm{fib}}\left(\theta^{2}=0\right) \approx 822.8 \frac{\mathrm{J}}{\mathrm{m}^{2}} \quad \text { and } \quad \Psi^{\mathrm{fib}}\left(\theta^{2}=b / 2\right)=\Psi^{\mathrm{fib}}\left(\theta^{2}=-b / 2\right) \approx 392.44 \frac{\mathrm{J}}{\mathrm{m}^{2}}
$$


and for the $\alpha=30^{\circ}$ fiber configuration

$$
\Psi^{\mathrm{fib}}\left(\theta^{2}=0\right) \approx 274.27 \frac{\mathrm{J}}{\mathrm{m}^{2}} \quad \text { and } \quad \Psi^{\mathrm{fib}}\left(\theta^{2}=b / 2\right)=\Psi^{\mathrm{fib}}\left(\theta^{2}=-b / 2\right) \approx 130.81 \frac{\mathrm{J}}{\mathrm{m}^{2}} .
$$

\section{References}

[1] R.A. Mindlin, Second gadient of strain and surface-tension in linear elasticity, Int. J. Solids Struct. 1 (1965) $417-438$.

[2] P. Germain, The method of virtual power in continuum mechanics. Part 2: Microstructure, SIAM J. Appl. Math. 25 (1973) $556-575$.

[3] R. Toupin, Elastic materials with couple-stresses, Arch. Ration. Mech. Anal. 11 (1962) 385-414.

[4] R.A. Toupin, Theories of elasticity with couple stress, Arch. Ration. Mech. Anal. 17 (1964) 85-112.

[5] A.C. Eringen, E.S. Suhubi, Nonlinear theory of simple microelastic solids, Internat. J. Engrg. Sci. 2 (1964) 189-203, 389-404.

[6] A.C. Eringen, D.G.B. Edelen, On nonlocal elasticity, Internat. J. Engrg. Sci. 10 (1972) 233-248.

[7] A.C. Eringen, Microcontinuum Field Theories I: Foundations and Solids, Springer, 1999.

[8] A. Eringen, G. Maugin, Electrodynamics of Continua I: Foundations and Solid Media, Springer, Dordrecht, Heidelberg, London, New York, 1990.

[9] G. Maugin, A. Metrikine, Mechanics of Generalized Continua. One Hundred Years After the Cosserats, Springer-Verlag New York, 2010.

[10] S. Forest, R. Sievert, Elastoviscoplastic constitutive frameworks for generalized continua, Acta Mech. 160 (2003) $71-111$.

[11] C. Hirschberger, E. Kuhl, P. Steinmann, On deformational and configurational mechanics of micromorphic hyperelasticity - Theory and computation, Comput. Methods Appl. Mech. Engrg. 196 (2007) 4027-4044.

[12] K. Saanouni, M. Hamed, Micromorphic approach for finite gradient-elastoplasticity fully coupled with ductile damage: Formulation and computational aspects, Int. J. Solids Struct. 50 (2013) 2289-2309.

[13] N. Kirchner, P. Steinmann, A unifying treatise on variational principles for gradient and micromorphic continua, Phil. Mag. 85 (2005) 3875-3895.

[14] A. Javili, F. dell'Isola, P. Steinmann, Geometrically nonlinear higher-gradient elasticity with energetic boundaries, J. Mech. Phys. Solids 61 (12) (2013) 2381-2401.

[15] P. Neff, The cosserat couple modulus for continuous solids is zero viz the linearized Cauchy-stress tensor is symmetric, ZAMM 86 (2006) 892-912.

[16] P. Neff, J. Jeong, A new paradigm: the linear isotropic cosserat model with conformally invariant curvature energy,, ZAMM 44 (2009) 574-594.

[17] F. Yang, A. Chong, D. Lam, P. Tong, Couple stress based strain gradient theory for elasticity, Int. J. Solids Struct. 39 (2002) $2731-2743$.

[18] D. Steigmann, A. Pipkin, Equilibrium of elastic nets, Philos. Trans. R. Soc. Lond. 335 (1991) 419-454.

[19] D. Steigmann, Theory of elastic solids reinforced with fibers resistant to extension, flexure and twist, Int. J. Non-Linear Mech. 47 (2012) 734-742.

[20] D. Steigmann, F. dell'Isola, Mechanical response of fabric sheets to three-dimensional bending, twisting, and stretching, Acta Mech. Sinica (2015).

[21] D. Steigmann, Equilibrium of elastic lattice shells, J. Eng. Math. 109 (2018) 47-61.

[22] F. dell'Isola, I. Giorgio, M. Pawlikowski, N.L. Rizzi, Large deformations of planar extensible beams and pantographic lattices: heuristic homogenization, experimental and numerical examples of equilibrium, Proc. R. Soc. A 472 (2185) (2016).

[23] F. dell'Isola, P. Seppecher, M. Spagnuolo, E. Barchiesi, F. Hild, T. Lekszycki, I. Giorgio, L. Placidi, U. Andreaus, M. Cuomo, S. Eugster, A. Pfaff, K. Hoschke, R. Langkemper, E. Turco, R. Sarikaya, A. Misra, M. De Angelo, F. D’Annibale, A. Bouterf, X. Pinelli, A. Misra, B. Desmorat, M. Pawlikowski, C. Dupuy, D. Scerrato, P. Peyre, M. Laudato, L. Manzari, P. Göransson, C. Hesch, S. Hesch, P. Franciosi, J. Dirrenberger, F. Maurin, Z. Vangelatos, C. Grigoropoulos, V. Melissinaki, M. Farsari, W. Muller, B. Abali, C. Liebold, G. Ganzosch, P. Harrison, R. Drobnicki, L. Igumnov, F. Alzahrani, T. Hayat, Advances in pantographic structures: design, manufacturing, models, experiments and image analyses, Contin. Mech. Thermodyn. 31 (2019) 1231-1282.

[24] J. Fish, Practical Multiscaling, John Wiley \& Sons, 2013.

[25] H. Wang, H. Zhou, J. Mishnaevsky, P. Brondsted, L. Wang, Single fibre and multifibre unit cell analysis of strength and cracking of unidirectional composites, Comput. Mater. Sci. 46 (2009) 810-820.

[26] L. Mishnaevsky, Computational Mesomechanics of Composites: numerical Analysis of the Effect of Microstructures of Composites of Strength and Damage Resistance, Wiley-Interscience, 2007.

[27] C.B. Hirschberger, A Treatise on Micromorphic Continua. Theory, Homogenization, Computation (Ph.D. thesis), Lehrstuhl für Technische Mechanik, Technische Universität Kaiserslautern, 2008.

[28] G. Hütter, Homogenization of a Cauchy continuum towards a micromorphic continuum, J. Mech. Phys. Solids 99 (2017) $394-408$.

[29] T. Asmanoglo, A. Menzel, A finite deformation continuum modelling framework for curvature effects in fibre-reinforced nanocomposites, J. Mech. Phys. Solids 107 (2017) 411-432.

[30] T. Asmanoglo, A. Menzel, A multi-field finite element approach for the modelling of fibre-reinforced composites with fibre-bending stiffness, Comput. Methods Appl. Mech. Engrg. 317 (2017) 1037-1067.

[31] A. Spencer, K. Soldatos, Finite deformations of fibre-reinforced elastic solids with fibre bending stiffness, Int. J. Non-Linear Mech. 42 (2007) 355-368.

[32] K. Soldatos, Second-gradient plane deformations of ideal fibre-reinforced materials: implications of hyper-elasticity theory, J. Eng. Math. 68 (2010) 99-127. 
[33] T. Hughes, J. Cottrell, Y. Bazilevs, Isogeometric analysis: CAD, finite elements, NURBS, exact geometry and mesh refinement, Comput. Methods Appl. Mech. Engrg. 194 (39-41) (2005) 4135-4195.

[34] J. Cottrell, T. Hughes, Y. Bazilevs, Isogeometric Analysis: Toward Integration of CAD and FEA, Wiley, 2009.

[35] P. Fischer, M. Klassen, J. Mergheim, P. Steinmann, R. Müller, Isogeometric analysis of 2D gradient elasticity, Comput. Mech. 47 (3) (2011) 325-334.

[36] J. Kiendl, K. Bletzinger, J. Linhard, R. Wüchner, Isogeometric shell analysis with Kirchhoff-Love elements, Comput. Methods Appl. Mech. Engrg. 198 (2009) 3902-3914.

[37] J. Kiendl, M. Hsu, M. Whu, A. Reali, Isogeometric Kirchhoff-Love shell formulations for general hyperelastic materials, Comput. Methods Appl. Mech. Engrg. 291 (2015) 280-303.

[38] V. Balobanov, J. Kiendl, S. Khakalo, J. Niiranen, Kirchhoff-Love shells within strain gradient elasticity: Weak and strong formulations and an $H^{3}$-conforming isogeometric implementation, Comput. Methods Appl. Mech. Engrg. 344 (2019) 837-857.

[39] P. Ciarlet, An Introduction to Differential Geometry with Aplications to Elasticity, Springer, 2005.

[40] S. Schuß, M. Dittmann, S. Klinkel, B. Wohlmuth, C. Hesch, Multi-patch isogeometric analysis for Kirchhoff-Love shell elements, Comput. Methods Appl. Mech. Engrg. 349 (2019) 91-116.

[41] P. Bornemann, F. Cirak, A subdivision-based implementation of the hierarchical b-spline finite element method, Comput. Methods Appl. Mech. Engrg. 253 (2013) 584-598.

[42] C. Hesch, S. Schuß, M. Dittmann, M. Franke, K. Weinberg, Isogeometric analysis and hierarchical refinement for higher-order phase-field models, Comput. Methods Appl. Mech. Engrg. 303 (2016) 185-207.

[43] M. Dittmann, Isogeometric analysis and hierarchical refinement for multi-field contact problems (Ph.D. thesis), University of Siegen, 2017.

[44] M. Ishaquddina, P. Raveendranath, J. Reddy, Flexure and torsion locking phenomena in out-of-plane deformation of Timoshenko curved beam element, Finite Elem. Anal. Des. 51 (2012) 22-30.

[45] F. Auricchio, L. Beirão da Veiga, J. Kiendl, C. Lovadina, A. Reali, Locking-free isogeometric collocation methods for spatial Timoshenko rods, Comput. Methods Appl. Mech. Engrg. 263 (2013) 113-126.

[46] L. Greco, M. Cuomo, An isogeometric implicit $G^{1}$ mixed finite element for Kirchhoff space rods, Comput. Methods Appl. Mech. Engrg. 298 (2016) 325-349.

[47] R. Echter, M. Bischoff, Numerical efficiency, locking and unlocking of NURBS finite elements, Comput. Methods Appl. Mech. Engrg. 199 (5-8) (2010) 374-382.

[48] K. Bletzinger, M. Bischoff, E. Ramm, A unified approach for shear-locking-free triangular and rectangular shell finite elements, Comput. Struct. 75 (3) (2000) 321-334.

[49] R. Bouclier, T. Elguedj, A. Combescure, Efficient isogeometric NURBS-based solid-shell elements: Mixed formulation and $\bar{B}$-method, Comput. Methods Appl. Mech. Engrg. 267 (2013) 86-110.

[50] L. Greco, M. Cuomo, L. Contrafatto, A reconstructed local $\bar{B}$ formulation for isogeometric Kirchhoff-Love shells, Comput. Methods Appl. Mech. Engrg. 332 (2018) 462-487.

[51] M. Dittmann, S. Schuß, B. Wohlmuth, C. Hesch, Weak $C^{n}$ coupling for multi-patch isogeometric analysis in solid mechanics, Internat. J. Numer. Methods Engrg. 118 (2019) 678-699.

[52] M. Dittmann, S. Schuß, B. Wohlmuth, C. Hesch, Crosspoint modification for multi-patch isogeometric analysis, Comput. Methods Appl. Mech. Engrg. 360 (2020) 112768.

[53] M. Dittmann, F. Aldakheel, J. Schulte, P. Wriggers, C. Hesch, Variational phase-field formulation of non-linear ductile fracture, Comput. Methods Appl. Mech. Engrg. 342 (2018) 71-94.

[54] M. Dittmann, F. Aldakheel, J. Schulte, F. Schmidt, M. Krüger, P. Wriggers, C. Hesch, Phase-field modeling of porous-ductile fracture in non-linear thermo-elasto-plastic solids, Comput. Methods Appl. Mech. Engrg. 361 (2020) 112730.

[55] F. Aldakheel, P. Wriggers, C. Miehe, A modified Gurson-type plasticity model at finite strains: Formulation, numerical analysis and phase-field coupling, Comput. Mech. 62 (4) (2017) 815-833.

[56] C. Hesch, P. Betsch, Transient 3D domain decomposition problems: Frame-indifferent mortar constraints and conserving integration, Internat. J. Numer. Methods Engrg. 82 (2010) 329-358.

[57] J. Marsden, T. Ratiu, Introduction to Mechanics and Symmetry, Springer, 2003. 